\title{
An Efficient Integrated Framework of Reliability- Based Robust Design Optimization for Computation-Intensive Structures
}

xiongming lai ( $\sim$ laixiongming@aliyun.com )

Huaqiao University

Ju Huang

Huaqiao University

Cheng Wang

Huaqiao University

Yong Zhang

Huaqiao University

\section{Research Article}

Keywords: reliability-based robust design optimization, performance measure approach, trust region, double-loop optimizations

Posted Date: May 7th, 2021

DOI: https://doi.org/10.21203/rs.3.rs-449748/v1

License: (9) This work is licensed under a Creative Commons Attribution 4.0 International License. Read Full License

Version of Record: A version of this preprint was published at Journal of Computational Design and Engineering on September 20th, 2022. See the published version at https://doi.org/10.1093/jcde/qwac096. 


\section{An Efficient Integrated Framework of Reliability-Based}

\section{Robust Design Optimization for Computation-Intensive}

\section{Structures}

Xiongming Lai*1, Ju Huang ${ }^{1}$, Cheng Wang ${ }^{2}$, Yong Zhang ${ }^{1}$

1 College of Mechanical Engineering and Automation, Huaqiao University, Xiamen, 361021, Republic of China

3 College of Computer Science and Technology, Huaqiao University, Xiamen, 361021, Republic of China

Correspondence information: Xiongming Lai, affiliation, College of Mechanical

Engineering and Automation, Huaqiao University, Xiamen, 361021, Republic of China, laixiongming@aliyun.com, 086-18876256280 


\title{
An efficient integrated framework of reliability-based robust
}

\section{design optimization for computation-intensive structures}

\author{
Xiongming Lai*1, Ju Huang ${ }^{1}$, Cheng Wang ${ }^{2}$, Yong Zhang ${ }^{1}$
}

1 College of Mechanical Engineering and Automation, Huaqiao University, Xiamen, 361021, Republic of China

3 College of Computer Science and Technology, Huaqiao University, Xiamen, 361021, Republic of China

Correspondence information: Xiongming Lai, affiliation, College of Mechanical Engineering and Automation, Huaqiao University, Xiamen, 361021, Republic of China, laixiongming@aliyun.com, 086-18876256280

\section{Abstract}

When carrying out robust design optimization for complex engineering structures, they are computed by finite element software and are always computation-intensive. Aim at this problem, the paper proposes an efficient integrated framework of Reliability-based Robust Design Optimization (RBRDO). Firstly, the conventional RBRDO problem is changed as percentile form, that is, the improved percentile formulation of computing the objective robustness and probabilistic constraints is presented by resorting to the employment of Performance Measure Approach (PMA). Secondly, the above improved RBRDO problem is simplified by a series of new approximation methods due to the need of reducing computation. An efficient approximation method is proposed to estimate PMA functions of the RBRDO formulation. Based on it, the above improved RBRDO problem can be transformed into 
An efficient integrated framework for reliability-based robust design optimization of computation-intensive structures

a sequence of approximate deterministic sub-optimization problems, whose objective function and constraints are expressed as the approximate explicit form only in relation to the design variables. Furthermore, use the trust region (TR) method to solve the above sequence of sub-optimization. Lastly, several examples are used to demonstrate the effectiveness and efficiency of the proposed method.

PACS(optional, as per journal): 75.40.-s; 71.20.LP

Keywords: reliability-based robust design optimization, performance measure approach, trust region, double-loop optimizations 
An efficient integrated framework for reliability-based robust design optimization of computation-intensive structures

\section{Introduction}

Reliability-based Robust Design Optimization (RBRDO) as the advanced method is wide-spread used in different fields of engineering applications(Fran et al., 2020; Gang et al., 2016; Hao et al., 2019; Panzeri et al., 2018; Papadimitriou \& Papadimitriou, 2016; Tsao \& Thanh, 2020). In RBRDO, the objective function or constraint function usually includes probability factors to consider the influence of uncertainty. In the probabilistic framework, the typical RBRDO problem is formulated as follows:

$$
\begin{aligned}
& \min \quad F(\mathbf{d}, \mathbf{X}, \mathbf{P}) \\
& \text { s.t: } \operatorname{Prob}\left\{G_{j}(\mathbf{d}, \mathbf{X}, \mathbf{P}) \leq 0\right\} \leq p_{f}^{j} \quad j=1 \ldots n \\
& \quad \mathbf{d}^{L} \leq \mathbf{d} \leq \mathbf{d}^{R}, \boldsymbol{\mu}_{\mathbf{X}}^{L} \leq \boldsymbol{\mu}_{\mathbf{X}} \leq \boldsymbol{\mu}_{\mathbf{X}}^{R}
\end{aligned}
$$

Within the over matrix, $F$ and $G_{j}$ are the execution capacities. $\mathbf{d} \in R^{k}$ is the vector of controllable deterministic design variables. $\mathbf{X} \in R^{m}$ is the vector of controllable arbitrary design variables, whose mean value $\mu_{\mathrm{x}}$ is controllable. $\mathbf{P} \in R^{q}$ is the vector of wild arbitrary design parameters or noise factors, whose mean value is uncontrollable. $n$ is the number of the constraints. Prob(.) refers to the probability of the $j$ th constraint. $p_{F}^{j}$ alludes to the failure of probability of the jth constraint. Superscripts "L" and "R" denote lower and upper limits, respectively.

Generally, the past literature on RBRDO can be classified into three different formulation approaches(Rathod et al., 2013). The first is the moment-based RBRDO formulation(Javed et al., 2019). It describes the robust design quality by using the statistical moments of a performance function (e.g. mean and variance). The second is the percentile difference-based RBRDO formulation(Castaldo et al., 2018). It obtains the percentile performance differences by comparing the right and left tails of the performance distribution and use it to approximately evaluate performance variation. 
Then it brings such performance variation into the objective function to describe the robustness. The third is hybrid quality-loss-functions-based RBRDO formulation(Ali et al., 2016). It endeavors to obtain the trade-off solutions that satisfy several constraints (objectives) to the extent possible. For all of the above methods, the uncertainties are quantified by conducting experimental design or Taylor series expansion or Monte Carlo simulation (MCS) and its variation-reduced methods. For most practical engineering problems, large-scale MCS and its variation-reduced methods are wildly used since they are simple and universal. However, MCS and its variation-reduced methods require to compute time-consuming functions for many times. Hence, serious computational obstacles are harmful to real-life engineering applications.

Eq. (1) shows that RBRDO is an double-loop optimization. The outer loop optimizes the design vector $\mathbf{d}$, while the inner loop determines the uncertainty of the objective function and constraints. In most engineering applications, each performance function always should be computed by large engineering software, e.g. the finite element software, and each time of computation is time-consuming. Therefore the repeated computation of performance functions required by MCS and its variation-reduced methods in the inner loop is more computation-intensive, let alone such repeated job of the inner loop due to the loop of the outer optimization. Aiming at this problem, scholars have put forward relevant research methods. $\mathrm{Gu}$ and $\mathrm{Lu}(\mathrm{Gu} \& \mathrm{Lu}, 2014)$ performed reliability-based robust design for occupant restraint system. They built the Support Vector Regression (SVR) surrogate by using the Optimal Latin Hypercube Sampling Technique (OLHST) and used the surrogate model to replace actual simulation model during the optimization process. Sun et al.(Sun et al., 2011) carried out crash worthiness design of the vehicle by using robust optimization. They used the OLHST and the dual 
response surface method to obtain the surrogate model, which were also used for robust optimization. Zhu (Zhu et al., 2009) et al. found SVR performs very well in highly nonlinear vehicle crash problems and used the surrogate model in the robust optimization process. Jaehyeok et al. built the thermoelastic-plasticity-creep model of pressurized water reactors annular fuels and combined the optimal Latin hypercube design and the kriging and inverse distance weighting method to obtain the surrogate model of the thermoelastic-plasticity-creep model, which greatly reduced the computation cost in the robust design optimization of the gap size of annular nuclear fuel(Doh et al., 2018). Gao et al. established the explicit mapping relationship between the casting process parameters and the casting quality by using the double-layer Kriging method and used the explicit model for relieving the computation when solving the robust optimal process parameters(Gao et al., 2020). Bohlouri et al. used quasinormalized equations to describe the second-order transfer response of the on-off thruster in spacecraft attitude control problem and build the surrogate model for reducing computational burden in reliability-based robust design optimization of spacecraft control(Bohlouri \& Jalali-Naini, 2019). It can be seen from the above literature that remarkable computation cost is saved by using an explicit cheap surrogate model to replace the actual time-consuming simulation model in the inner loop of robust optimization. In any case, there exists two problems. On one hand, the minimal sampling size for building the approximate surrogate model is always difficult to determine. When the sampling size is too large, the computation times of the actual simulation model is correspondingly increased and the computation cost become expensive. Otherwise, the accuracy of the constructed surrogate model is compromised. On the other hand, the job of constructing the surrogate model in the inner loop is not 
once-and-done operation. Such job should be frequently repeated once the iterative design vector in the outer loop is frequently updated.

Aiming at the above two problems, this paper develops a new efficient integrated framework for the RBRDO. Firstly, an improved percentile difference-based formulation of the RBRDO is presented. Then the improved formulation of RBRDO can be changed as a sequence of approximate deterministic sub-optimization problems, whose boundaries of design vector $\mathbf{d}$ are limited in the narrow ranges. In this way, the times of constructing the surrogate model by the sampling simulation are greatly reduced, since the Taylor series expansion can be used to modified the surrogate model (See section 3.1). Correspondingly, the total computation times of actual simulation model are also greatly reduced. Meanwhile, such modification avoid the difficulty of determining the sampling size. Then the TR method is managed to solving the above sequence of sub-optimization problems and during the solving process the boundaries of design vector $\mathbf{d}$ for each sub-optimization problems are adjusted to make the constructed explicit surrogate model accurate enough within the specified sampling size. Hence, the above two problems can be solved to a large extent.

The paper is organized as follows: Section 2 introduces the basic theory of RBRDO and presents an improved RBRDO formulation. Section 3 presents a series of approximation methods for RBRDO. In Section 4 analyzes a benchmark test and two practical engineering problems to show the effectiveness and efficiency of the presented method. Section 5 concludes the paper.

\section{The formulation of the RBRDO}




\subsection{The general formulation of the RBRDO}

Generally, Eq. (1) is usually written in the form of the Weighted Sum (WS):

$$
\begin{aligned}
& \min \quad W=(1-\alpha) \mu_{F} / \phi+\alpha \sigma_{F} / \varphi \\
& \text { s.t: } P\left\{G_{j}(\mathbf{d}, \mathbf{X}, \mathbf{P}) \leq 0\right\} \leq p_{f}^{j} \quad j=1 \ldots n \\
& \mathbf{d}^{L} \leq \mathbf{d} \leq \mathbf{d}^{R}, \boldsymbol{\mu}_{\mathbf{X}}^{L} \leq \boldsymbol{\mu}_{\mathbf{X}} \leq \boldsymbol{\mu}_{\mathbf{X}}^{R}
\end{aligned}
$$

where $\mu_{F}$ and $\sigma_{F}$ denotes the mean and standard deviation of the performance function $F(\mathbf{d}, \mathbf{X}, \mathbf{P})$, respectively. $\alpha \in[0,1]$ is the weighting factor. $\phi$ and $\varphi$ are normalization factors of $\mu_{F}$ and $\sigma_{F}$.

In order to apply the presented method, the deterministic design vector $\mathbf{d}$ is assumed as normal random one with its variations limited in very narrow boundaries. Apparently, the scatter of the performance function, i.e. $F$ and $G_{j}$ caused by the variations of the design vector $\mathbf{d}$ can be ignored, when the variation boundaries of the design vector $\mathbf{d}$ are set as tiny values. Hence, the optimal solutions of RBRDO under such treatment can approximate the solutions of original problem. Besides, it helps to combine the original design vector $\mathbf{d}$ and the original random vector as random ones and brings great convenience for mathematical processing in RBRDO. Based on the simulation investigations, a small value of the standard deviation $\boldsymbol{\sigma}_{\mathbf{d}}$ of the design vector d is suggested and the optimization results are not sensitive to the small value.

For sake of discussion, $\mathbf{d}, \mathbf{X}$ and $\mathbf{P}$ are combined as $\mathbf{U}=[\mathbf{d}, \mathbf{X}, \mathbf{P}]^{T}$, and its mean and standard deviation vectors are written as $\boldsymbol{\mu}_{\mathbf{U}}=\left[\boldsymbol{\mu}_{\mathbf{d}}, \boldsymbol{\mu}_{\mathbf{X}}, \boldsymbol{\mu}_{\mathbf{P}}\right]^{T}$ and $\boldsymbol{\sigma}_{\mathbf{U}}=\left[\boldsymbol{\sigma}_{\mathbf{d}}, \boldsymbol{\sigma}_{\mathbf{X}}, \boldsymbol{\sigma}_{\mathbf{P}}\right]^{T}$, respectively. For the sake of focusing on stating the method introduced in this paper, all random variables in $\mathbf{U}$ are assumed as independent and normal. As for correlated arbitrary random variables, they can be transformed into 
normal independent ones by Rosenblatt transformation(Rosenblatt, 1952). Meanwhile, $\boldsymbol{\mu}_{\mathbf{d}}$ and $\boldsymbol{\mu}_{\mathbf{x}}$ are combined as $\mathbf{V}=\left[\boldsymbol{\mu}_{\mathbf{d}}, \boldsymbol{\mu}_{\mathbf{x}}\right]^{T}$. Correspondingly, $\mathbf{V}^{L}$ and $\mathbf{V}^{R}$ denote lower and upper limits, respectively. Then, Eq. (2) is:

$$
\begin{array}{cc}
\min & W=(1-\alpha) \mu_{F} / \phi+\alpha \sigma_{F} / \varphi \\
\text { s.t: } & P\left\{\left(G_{j}(\mathbf{U} ; \mathbf{V}) \leq 0\right\} \leq p_{f}^{j} \quad j=1 \ldots n\right. \\
& \mathbf{V}^{L} \leq \mathbf{V} \leq \mathbf{V}^{R}
\end{array}
$$

In U-space (the independent and standardized normal space), $\mathbf{U}$ denotes the standardized normal vector $\mathbf{u}$ and the relationship between the components in $\mathbf{U}$ and those in $\mathbf{u}$ are as follows:

$$
u_{i}=\left(U_{i}-\mu_{U_{i}}\right) / \sigma_{U_{i}} \quad i=1, \ldots, k, k+1, \ldots, k+m, k+m+1, \ldots, k+m+q
$$

Then Eq. (3) can be further changed to:

$$
\begin{array}{cc}
\min & W=(1-\alpha) \mu_{f} / \phi+\alpha \sigma_{f} / \varphi \\
\text { s.t: } & P\left\{\left(g_{j}(\mathbf{u} ; \mathbf{V}) \leq 0\right\} \leq p_{f}^{j} \quad j=1 \ldots n\right. \\
& \mathbf{V}^{L} \leq \mathbf{V} \leq \mathbf{V}^{R}
\end{array}
$$

where $g_{j}(\mathbf{u} ; \mathbf{V}) \equiv G_{j}(\mathbf{U} ; \mathbf{V}), \quad f(\mathbf{u} ; \mathbf{V}) \equiv F(\mathbf{U} ; \mathbf{V})$.

Fig. 1 outlines the general procedure to solve Eq. (5). As Fig. 1 shows, it necessitates the frequent job of building the surrogate models by the sampling simulation in the inner loop optimization due to the frequent update of the design vector in the outer loop optimization. Hence, the total evaluation number of actual model simulation is quite large. In the following paper, an efficient integrated framework of RBRDO is presented. It attaches importance to greatly reduce the repeated job of building the surrogate models by the sampling simulation. 


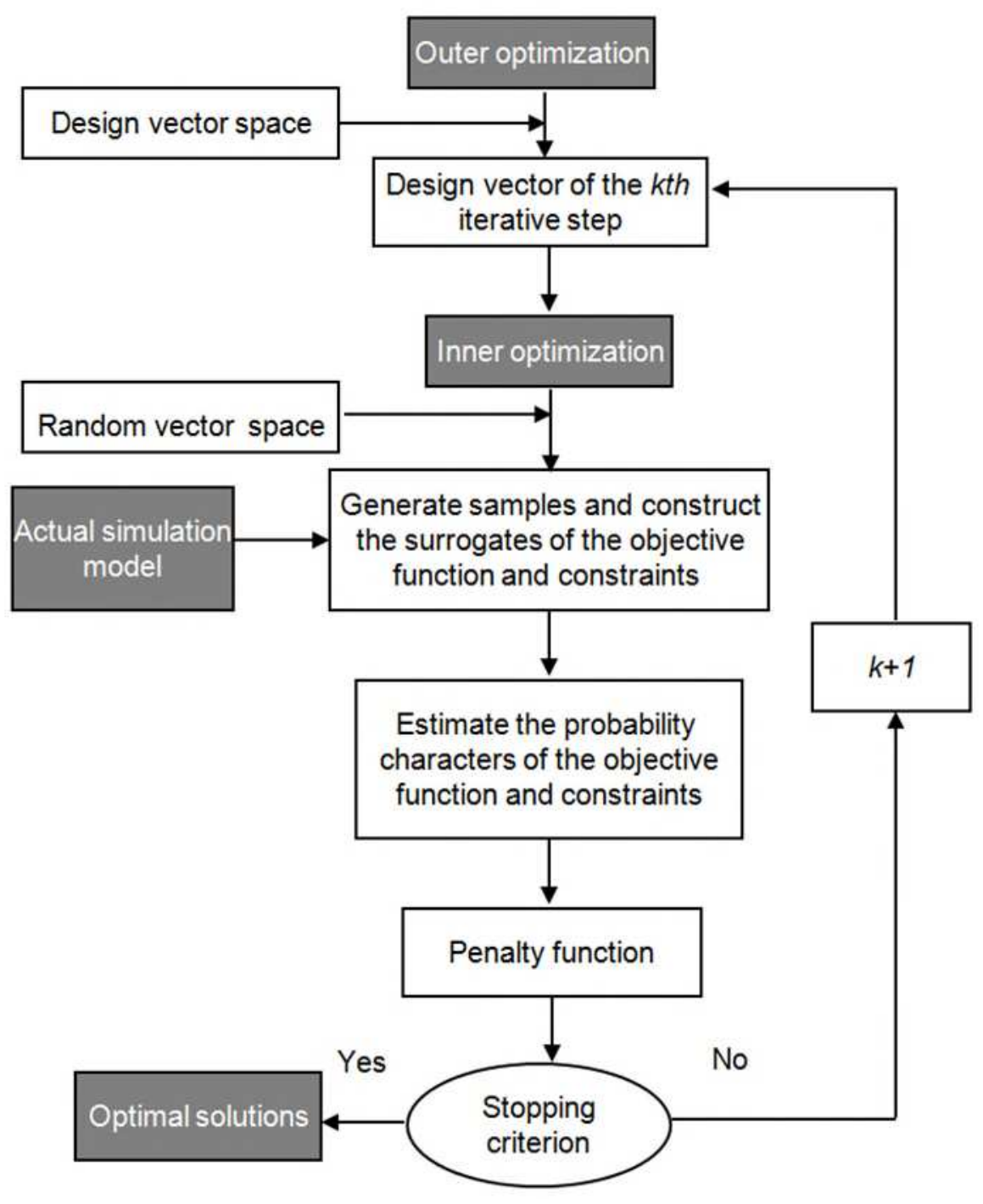

Fig. 1. The procedure of double-loop optimization for the general RBRDO.

\subsection{Improvement of the RBRDO formulation}

For the convenience of applying the approximate methods for RBRDO, PMA is used to improve the RBRDO formulation. PMA can be used to describe the probabilistic uncertainty of the performance function. It solves the following optimization problem(Hao et al., 2019)(see Eq. (6)) to judge whether a design vector meets the probabilistic constraint corresponding to a given reliability index $\beta_{j}$ : 


$$
\begin{array}{ll}
\min & g_{j}(\mathbf{u} ; \mathbf{V}) \\
\text { s.t: } & \|\mathbf{u}\|=\beta_{j}
\end{array}
$$

where $\beta_{j}=\Phi^{-1}\left(1-p_{F}^{j}\right) . \Phi^{-1}$ is the inverse cumulative distribution function of the standard normal distribution. The optimum point of Eq. (6) is called the MPP and denoted by $\mathbf{u}_{j}^{*}$ in U-space. Then the reliability index $\beta_{j}$ denotes the minimum distance from the origin to the MPP.

Further, the original probabilistic constraint function, $P\left\{g_{j}(\mathbf{u} ; \mathbf{V}) \leq 0\right\} \leq p_{f}^{j}$, can be changed as the deterministic function, $g_{j}\left(\mathbf{u}_{j}^{*} ; \mathbf{V}\right) \geq 0$.

With the help of PMA, the objective function in Eq. (5) can be represented by Percentile Difference Method (PDM)(Lee et al., 2008). Fig. 2 shows the probability density distribution of the performance function $f, f_{p 0.5}$ is the mean value of the performance function $f$, i.e. $\mu_{f} . p_{1}$ and $p_{2}$ are the left-tail and right-tail percentiles, respectively. Meanwhile, $p_{1}+p_{2}=1$. In this paper, the reliability index $\beta_{p 1}$ for $p_{1}$ and the reliability index $\beta_{p 2}$ for $p_{2}$ are set as -3 and 3 , respectively. Then $p_{1}=1-\Phi\left(\beta_{p 1}\right) \approx 0.00135$ and $p_{2}=1-\Phi\left(\beta_{p 2}\right) \approx 0.99865$. It implies that the probability of shaded area (See Fig. 2) lied between $p_{1}$ and $p_{2}$ is 0.9973.

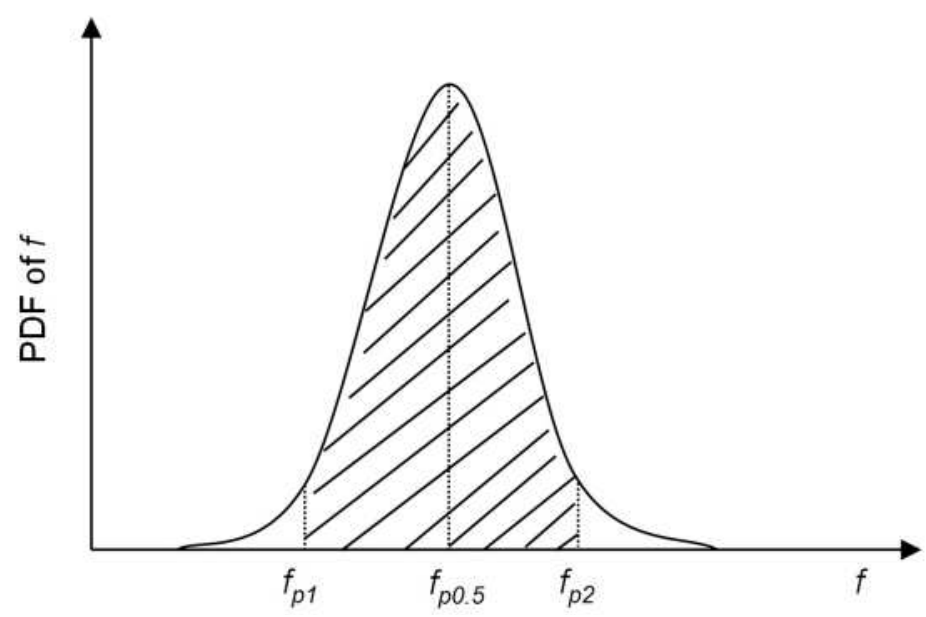


Fig. 2 The distribution of the performance function $f$.

By resorting to PMA, $f_{p 1}$ and $f_{p 2}$ can be represented as:

$$
\begin{array}{r}
f_{p 1}=f\left(\mathbf{u}_{p 1}^{*} ; \mathbf{V}\right) \\
f_{p 2}=f\left(\mathbf{u}_{p 2}^{*} ; \mathbf{V}\right)
\end{array}
$$

where $\mathbf{u}_{p 1}^{*}$ and $\mathbf{u}_{p 2}^{*}$ are the solutions of Eq. (8) and Eq. (9), respectively.

$$
\begin{array}{ll}
\min & f(\mathbf{u} ; \mathbf{V}) \\
\text { s.t: } & \|\mathbf{u}\|=\beta_{f} \\
& \\
\max & f(\mathbf{u} ; \mathbf{V}) \\
\text { s.t: } & \|\mathbf{u}\|=\beta_{f}
\end{array}
$$

For the sake of convenient statement in the following paper, $f_{p 1}, f_{p 2}$ and $g_{j}\left(\mathbf{u}_{j}^{*} ; \mathbf{V}\right)$ are called PMA function, since they are obtained by PMA.

There are several advantages of using the PDM (i.e. $f_{p 2}-f_{p 1}$ ) to replace the general standard deviation $\sigma_{f}$ in Eq. (3) for robustness assessment (Du et al., 2004), but it's not applicable to the case where the performance function is not monotonic(Lee et al., 2008). For example, if $F(\boldsymbol{u})=\boldsymbol{u}^{2}[\boldsymbol{u} \sim \boldsymbol{N}(0,1)]$ and $\beta_{f}$ is 3, then two MPPs become 1.732 and -1.732 . Thus, the percentile performance difference (i.e. $f_{p 2}-f_{p 1}$ ) is 0 . To assessing the robustness of the performance function $f$, the following improvement of PDM takes into account three characteristic values, i.e. $f_{p 0}, f_{p 1}$ and $f_{p 2}$, as shown in Eq. (10).

$$
\min \left[\mu_{f}, \max \left(\left|f_{p 1}-\mu_{f}\right|,\left|f_{p 2}-\mu_{f}\right|\right)\right]
$$

Through over steps, the RBRDO form of Eq. (5) can be changed as: 


$$
\begin{gathered}
\min \quad W=\alpha \mu_{f} / \phi+(1-\alpha) \Delta_{f} / \varphi \\
\text { s.t: } g_{j}\left(\mathbf{u}_{j}^{*} ; \mathbf{V}\right) \geq 0 \quad j=1 \ldots n \\
\Delta_{f}=\max \left(\left|f_{p 1}-\mu_{f}\right|,\left|f_{p 2}-\mu_{f}\right|\right) \\
f_{p 1}=f\left(\mathbf{u}_{p 1}^{*} ; \mathbf{V}\right) \\
f_{p 2}=f\left(\mathbf{u}_{p 2}^{*} ; \mathbf{V}\right) \\
\mathbf{V}^{L} \leq \mathbf{V} \leq \mathbf{V}^{R}
\end{gathered}
$$

Using penalty function method, Eq. (11) can be further formulated to:

$$
\begin{aligned}
\min H_{p} & =W+\kappa \sum_{j=1}^{n} \max \left(0,-g_{j}\left(\mathbf{u}_{j}^{*} ; \mathbf{V}\right)\right) \\
W & =\alpha \mu_{f} / \phi+(1-\alpha) \Delta_{f} / \varphi \\
\Delta_{f} & =\max \left(\left|f_{p 1}-\mu_{f}\right|,\left|f_{p 2}-\mu_{f}\right|\right) \\
f_{p 1} & =f\left(\mathbf{u}_{p 1}^{*} ; \mathbf{V}\right) \\
f_{p 2} & =f\left(\mathbf{u}_{p 2}^{*} ; \mathbf{V}\right) \\
\mathbf{V}^{L} & \leq \mathbf{V} \leq \mathbf{V}^{R}
\end{aligned}
$$

where $\kappa$ is a penalty factor.

By slightly modifying the procedure of Fig. 1, Eq. (12) can be solved, as shown in Fig. 3. Like the general formulation shown in Eq. (5), Eq. (12) is also a double-loop optimization problem. As showed in Fig. 3, the outer loop optimizes the design vector $\mathbf{V}$ and the inner loop solves the uncertainties of the performance functions. It should be noted that, it needs to compute the actual simulation model for a certain number of times for building the surrogate model which can be used to evaluate the performance functions in the inner loop. And such job should be repeated once the outer design vector is updated. Hence, the number of computing the actual simulation model very large. Due to this disadvantage, the paper proposes the approximation methods for RBRDO to greatly decrease the evaluation number of the actual simulation model in the following paper. 
An efficient integrated framework for reliability-based robust design optimization of computation-intensive structures

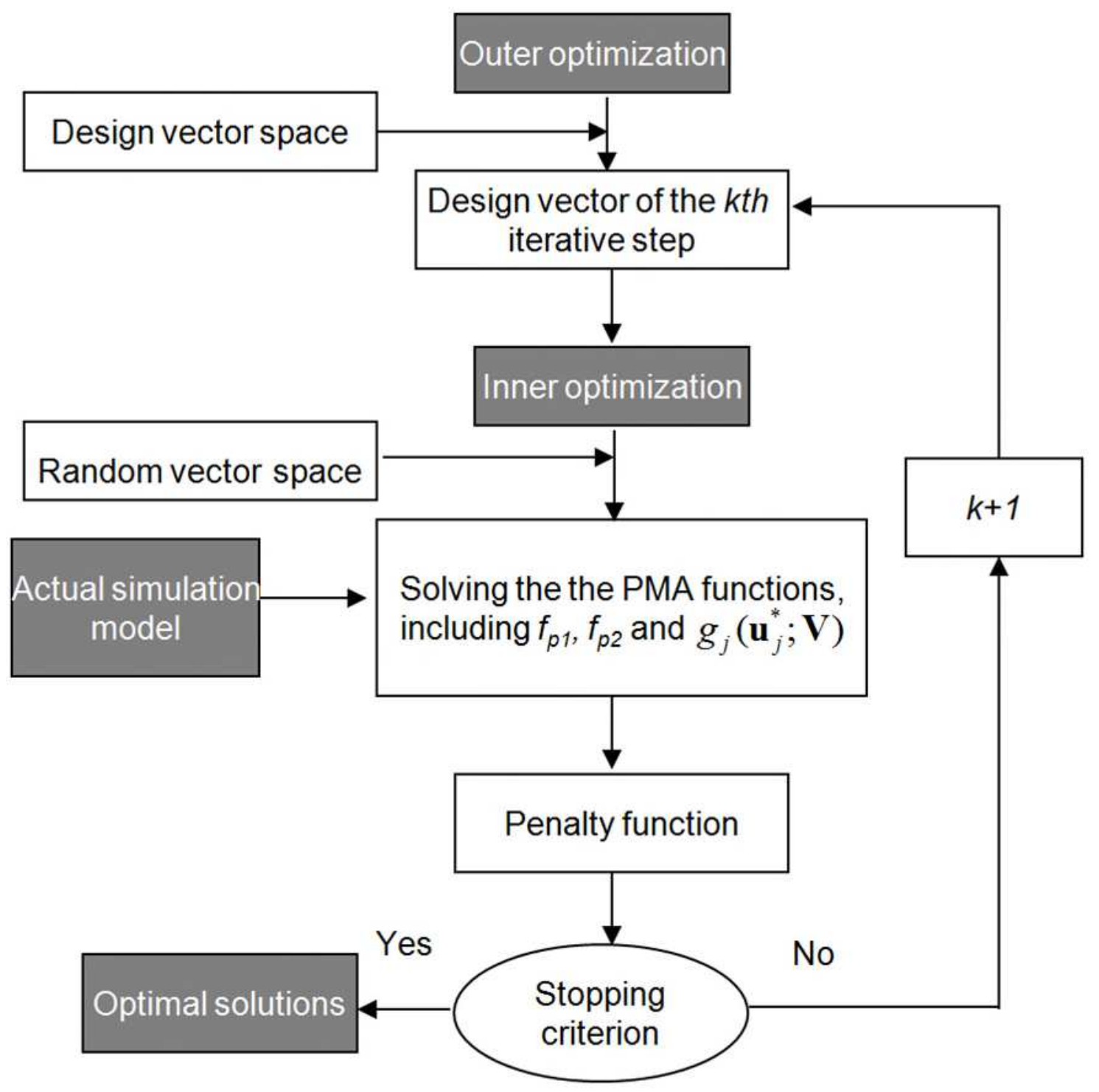

Fig. 3. The procedure of the double-loop optimization for the improved RBRDO formulation.

\section{Approximation methods for RBRDO}

\subsection{Approximate estimation of the PMA function}

In Eq. (12), it necessitates to work out the MPP to obtain the response value of the PMA functions, i.e. $f_{p 1}, f_{p 2}$ and $g_{j}\left(\mathbf{u}_{j}^{*} ; \mathbf{V}\right)$. As for the arbitrary PMA function $K$, the MPP on the disappointment surface in U-space is computed as follows(Ang \& Tang, 1984; Yang et al., 2020): 


$$
u_{i}^{*}=-\frac{\left(\partial K / \partial u_{i}\right)_{*}}{\sqrt{\sum_{i}\left(\partial K / \partial u_{i}\right)_{*}^{2}}} \beta_{j}
$$

where $u_{i}^{*}$ is the chid element of $\mathbf{u}^{*}$, and $\left(\partial K / \partial u_{i}\right)_{*} / \sqrt{\sum_{i}\left(\partial K / \partial u_{i}\right)_{*}^{2}}$ is the direction cosine along the axis $u_{i}$.

According to Eq. (4), $\partial K / \partial u_{i}$ can be rewritten as :

$$
\frac{\partial K}{\partial u_{i}}=\frac{\partial K}{\partial U_{i}} \frac{\partial U_{i}}{\partial u_{i}}=\sigma_{U_{i}} \frac{\partial K}{\partial U_{i}}
$$

Combining Eq. (4) and Eq. (14), Eq. (13) can be transformed as Eq. (15) within the original design space (Ang \& Tang, 1984):

$$
U_{i}^{*}=\mu_{U_{i}}-\left.\beta_{j} \sigma_{U_{i}}^{2} \frac{\left(\partial K / \partial U_{i}\right)}{\sqrt{\sum_{i}\left(\sigma_{U_{i}}\left(\partial K / \partial U_{i}\right)\right)^{2}}}\right|_{U_{i}=U_{i}^{*}}
$$

It is noted that $\boldsymbol{\mu}_{\mathbf{U}}=\left[\mathbf{V}, \boldsymbol{\mu}_{\mathbf{P}}\right]$. From Eq. (12) and Eq. (15), the realization value of the design vector $\mathbf{V}$ is corresponding to a specific value of the MPP $\mathbf{U}^{*}$. Hence, $\mathbf{U}^{*}$ is changed with the updated $\mathbf{V}$ during the optimization process. To obtain the MPP $\mathbf{U}^{*}$, an iterative strategy(Rackwitz \& Flessler, 1978) is required since it is within the both sides of Eq. (15). But by doing so, it increases the computation times of the PMA function. Instead of iteratively computing the MPP $\mathbf{U}^{*}$, the paper approximates it by using the mean point:

$$
U_{i}^{*} \approx \mu_{U_{i}}-\left.\beta_{j} \sigma_{U_{i}}^{2} \frac{\left(\partial K / \partial U_{i}\right)}{\sqrt{\sum_{i}\left(\sigma_{U_{i}}\left(\partial K / \partial U_{i}\right)\right)^{2}}}\right|_{U_{i}=\mu_{U_{i}}}
$$

In order to compute $\partial K / \partial \mu_{U_{i}}$ and simultaneously minimize the evaluation number of the PMA function, only $\mathrm{M}(=\mathrm{k}+\mathrm{m}+\mathrm{q})+1$ interpolation points are chosen as:

$$
\mathbf{y}_{i}=\left\{\begin{array}{lr}
\left(\mu_{1}, \mu_{2}, \ldots, \mu_{i-1}, U_{i}, \mu_{i+1}, \ldots, \mu_{M}\right) & i=1, \ldots, M \\
\left(\mu_{1}, \mu_{2}, \ldots, \mu_{M}\right) & i=M+1
\end{array}\right.
$$


where $U_{i}=\mu_{i}+c \sigma_{i}, c$ is a small value and the smaller the better.

Resorting to Eq. (17), $\partial K / \partial \mu_{U_{i}}$ can be expressed in the finite difference form as:

$$
\frac{\partial K}{\partial U_{i}} \approx \frac{K\left(\mathbf{y}_{i} ; \mathbf{V}\right)-K\left(\mathbf{y}_{M+1} ; \mathbf{V}\right)}{c \sigma_{i}}
$$

Based on Eqs. (16)-(18), the MPP $\mathbf{U}^{*}$ and its corresponding PMA function $K\left(\mathbf{U}^{*} ; \mathbf{V}\right)$ can be approximately estimated with the change of the updated $\mathbf{V}$ during the optimization process.

It is worth noticing that when the PMA function $K\left(\mathbf{U}^{*} ; \mathbf{V}\right)$ is constant, monotonously increasing, or monotonously increasing, the above method can precisely approximate its real value. However, if the PMA function is non-monotonical, it is difficult to search the MPP near the saddle points and the approximate value of the PMA function $K\left(\mathbf{U}^{*} ; \mathbf{V}\right)$ has a larger error. This disadvantage is ubiquitous for all MPP-based methods(Shan \& Wang, 2008). As a result, when using the above method, it should be given full attention for the non-monotonical cases.

In fact, $K\left(\mathbf{U}^{*} ; \mathbf{V}\right)$ is the function only with respect the design vector $\mathbf{V}$. Hence, $\mathbf{U}^{*}$ in the $K\left(\mathbf{U}^{*} ; \mathbf{V}\right)$ can be ignored and $K\left(\mathbf{U}^{*} ; \mathbf{V}\right)$ is abbreviated as $K(\mathbf{V})$ in the following paper.

Clearly, the frequent update of $\mathbf{V}$ increases the sampling simulation and the assessment number of the performance function. But if the new update of $\mathbf{V}$, says $\mathbf{V}_{\text {new }}$, is in the vicinity of $\mathbf{V}$, repeated construction of the corresponding performance function $K\left(\mathbf{V}_{\text {new }}\right)$ by the sampling simulation can be avoided by resorting to the Taylor series expansion. 


$$
K\left(\mathbf{V}_{\text {new }}\right)=K(\mathbf{V})+\frac{\partial K}{\partial \mathbf{V}}\left(\mathbf{V}_{\text {new }}-\mathbf{V}\right)^{T}
$$

where the components of $\partial K / \partial \mathbf{V}$ is derived as follows:

$$
\frac{\partial K}{\partial V_{i}}=\frac{\partial K}{\partial \mu_{U_{i}}}=\frac{\partial K}{\partial U_{i}} \frac{\partial U_{i}}{\partial \mu_{U_{i}}}=-\frac{1}{\sigma_{U_{i}}} \frac{\partial K}{\partial U_{i}} \quad(i=1, \ldots, k+m)
$$

With the help of Eqs. (17)-(20), the PMA functions in Eq. (12) are expressed as the explicit function only in relation to the design vector $\mathbf{V}$. By using such explicit function, the computation times for the performance function can be greatly decreased. To this end, the sequential approximate optimization for the RBRDO is presented.

\subsection{Sequential approximate RBRDO}

In order to ease the frequent update of the design vector during the optimization process, a new sequence of approximate optimizations is suggested to replace the original optimization and the TR method is used to ensure the solutions of the new sequence of optimizations to converge to the original ones.

In the optimization process, a sequence of approximate optimizations is constructed. At the kth iterative step, the sequence optimization for Eq. (11) can be written as:

$$
\begin{array}{ll}
\min & W=\alpha \mu_{f} / \phi+(1-\alpha) \Delta_{f} / \varphi \\
\text { s.t: } & g_{j}\left(\mathbf{U}_{j}^{*} ; \mathbf{V}\right) \geq 0 \quad j=1 \ldots n \\
& \Delta_{f}=\max \left(\left|f_{p 1}-\mu_{f}\right|,\left|f_{p 2}-\mu_{f}\right|\right) \\
& f_{p 1}=f\left(\mathbf{U}_{p 1}^{*} ; \mathbf{V}\right) \\
& f_{p 2}=f\left(\mathbf{U}_{p 2}^{*} ; \mathbf{V}\right) \\
& \max \left[\mathbf{V}^{L}, \mathbf{V}^{k}-\boldsymbol{\delta}^{k}\right] \leq \mathbf{V} \leq \min \left[\mathbf{V}^{R}, \mathbf{V}^{k}+\boldsymbol{\delta}^{k}\right]
\end{array}
$$


where $\mu_{f}^{r}, \boldsymbol{\delta}^{k}$ and $\mathbf{V}^{k}$ are the mean values of $f$, the TR radius vector and the current design vector at the $k$ th iterative step, respectively. $\boldsymbol{\delta}^{k}$ changes with the iterative step. If $\boldsymbol{\delta}^{k}$ is a small enough interval, the updated $\mathbf{V}$ is in the vicinity of $\mathbf{V}^{k}$. Then it is possible to use the local explicit function suggested in Section 3.1 to approximate the PMA values in Eq. (21). The following approximate optimization model for Eq. (21) is changed as:

$$
\begin{array}{ll}
\min & \tilde{W}(\mathbf{V})=\alpha \mu_{f} / \phi+(1-\alpha) \tilde{\Delta}_{f} / \varphi \\
\text { s.t: } & \tilde{g}_{j}(\mathbf{V}) \geq 0 \quad j=1 \ldots n \\
& \tilde{\Delta}_{f}=\max \left(\left|\tilde{f}_{p 1}(\mathbf{V})-\mu_{f}\right|,\left|\tilde{f_{p 2}}(\mathbf{V})-\mu_{f}\right|\right) \\
& \max \left[\mathbf{V}^{L}, \mathbf{V}^{k}-\boldsymbol{\delta}^{k}\right] \leq \boldsymbol{\mu}_{\mathbf{d}} \leq \min \left[\mathbf{V}^{R}, \mathbf{V}^{k}+\boldsymbol{\delta}^{k}\right]
\end{array}
$$

where $\tilde{f}_{p 1}(\mathbf{V}), \tilde{f_{p 2}}(\mathbf{V})$ and $\tilde{g}_{j}(\mathbf{V})$ are approximate explicit representations only with respect to the design vector around a current design $\mathbf{V}$. Correspondingly, $\Delta_{f}$ and $W(\mathbf{V})$ computed by the above approximate PMA functions are also written as $\tilde{\Delta}_{f}$ and $\tilde{W}(\mathbf{V})$, respectively. Obviously, Eq. (22) is the deterministic optimization and it can be further changed into the following penal format like Eq. (23):

$$
\begin{gathered}
\min \quad \tilde{H}_{p}(\mathbf{V})=\tilde{W}(\mathbf{V})+\kappa \sum_{j=1}^{n} \max \left(0,-\tilde{g}_{j}(\mathbf{V})\right) \\
\tilde{W}(\mathbf{V})=\alpha \mu_{f} / \phi+(1-\alpha) \tilde{\Delta_{f}} / \varphi \\
\tilde{\Delta_{f}}=\max \left(\left|\tilde{f_{p 1}}(\mathbf{V})-\mu_{f}\right|,\left|\tilde{f_{p 2}}(\mathbf{V})-\mu_{f}\right|\right) \\
\max \left[\mathbf{V}^{L}, \mathbf{V}^{k}-\boldsymbol{\delta}^{k}\right] \leq \mathbf{V} \leq \min \left[\mathbf{V}^{R}, \mathbf{V}^{k}+\boldsymbol{\delta}^{k}\right]
\end{gathered}
$$

where $\tilde{H}_{p}(\mathbf{V})$ (termed as "approximate penalty function") is computed also based on the above approximate PMA functions. And particle swarm optimization (PSO) is 
used to solve Eq. (23) in this paper, since it possesses a fine global convergence performance and has been widely used in an amount of literature(Dimopoulos, 2007; Praveen \& Duvigneau, 2009).

PSO updates the current position of each particle in the swarm by adjusting the velocity vector:

$$
\begin{aligned}
& \mathbf{v}_{i}^{h+1}=\omega \mathbf{v}_{i}^{h}+c_{1} \delta_{1}\left(\mathbf{P}_{i}^{h}-\mathbf{x}_{i}^{h}\right)+c_{2} \delta_{2}\left(\mathbf{G}_{i}^{h}-\mathbf{x}_{i}^{h}\right) \\
& \mathbf{x}_{i}^{h+1}=\mathbf{x}_{i}^{h}+\mathbf{v}_{i}^{h}
\end{aligned}
$$

where $\mathbf{v}_{i}^{h}$ and $\mathbf{x}_{i}^{h}$ are the velocity vector and the location vector of granule $i$ at iteration $h$, separately. $\mathbf{P}_{i}^{h}$ is the personal best location vector of particle $i . \mathbf{G}_{i}^{h}$ is the global best location vector of the whole swarm. $c_{1}$ and $c_{2}$ are the learning factors. $\delta_{1}$ and $\delta_{2}$ are random numbers uniformly distributed between 0 and 1. $\omega$ is inertia weight. For the examples illustrated in this paper, $\omega=0.7298, c_{1}=c_{2}=1.4962$. Please read Section 2.2 in (Wu et al., 2011) for more information about the PSO. Since Eq. (23) is the deterministic optimization and the actual model simulation is avoided, the computational effort required by PSO is acceptable.

In order to ensure the approximate accuracy between Eq. (12) and Eq. (23), the side constraints for the design vector $\mathbf{V}$ in Eq. (23) should be modified at each optimization cycle according to certain strategy. In this paper, the TR method as the strategy is used. At the $k$ th iterative step, it adjusts $\boldsymbol{\delta}^{k}$ by the criterion, that is current optimization in Eq. (23) has good approximation to the original optimization problem.

Such good approximation is quantified by the approximate degree of the penalty function in this paper. Hence, the trust index containing the information of the penalty function is defined as follow: 


$$
\rho^{k}=\frac{H_{p}\left(\mathbf{V}^{k}\right)-H_{p}\left(\mathbf{V}^{* k}\right)}{H_{p}\left(\mathbf{V}^{k}\right)-\tilde{H}_{p}\left(\mathbf{V}^{* k}\right)}
$$

where $\mathbf{V}^{* k}$ is the optimum for the $k t h$ approximation optimization problem in Eq. (23).

In Eq. (26), the approximate penalty function $\tilde{H}_{p}$ can be obtained by solving Eq. (23). And it also necessitates the computation of $H_{p}$, which denotes the actual value of the penalty function (termed as "actual penalty function") and is always unknown and implicit for most of the complex engineering problems.

In general, there are many TR methods with respect to the update of the TR radius for sequential approximate optimization(Conn et al., 1988). They are widely used in engineering optimization due to their good convergence. In this paper, the updating strategy shown in Eq. (27) is used(Jiang et al., 2008; Rodriguez et al., 1998):

$$
\left\{\begin{array}{l}
\boldsymbol{\delta}^{k+1}=0.5 \boldsymbol{\delta}^{k}, \mathbf{V}^{k+1}=\mathbf{V}^{k} \quad \text { if } \rho^{k} \leq 0 \\
\boldsymbol{\delta}^{k+1}=0.5 \boldsymbol{\delta}^{k}, \mathbf{V}^{k+1}=\mathbf{V}^{* k} \quad \text { if } 0 \leq \rho^{k} \leq 0.25 \\
\boldsymbol{\delta}^{k+1}=\boldsymbol{\delta}^{k}, \mathbf{V}^{k+1}=\mathbf{V}^{* k} \quad \text { if } \quad 0.25 \leq \rho^{k} \leq 0.75 \\
\boldsymbol{\delta}^{k+1}=2 \boldsymbol{\delta}^{k}, \mathbf{V}^{k+1}=\mathbf{V}^{* k} \quad \text { if } \rho^{k} \geq 0.75 \text { and }\left\|\mathbf{V}^{* r}-\mathbf{V}^{r+1}\right\|=\boldsymbol{\delta}^{k} \\
\boldsymbol{\delta}^{k+1}=\boldsymbol{\delta}^{k}, \mathbf{V}^{k+1}=\mathbf{V}^{* k} \quad \text { if } \rho^{k} \geq 0.75 \text { and }\left\|\mathbf{V}^{* k}-\mathbf{V}^{k+1}\right\| \mathrm{p} \boldsymbol{\delta}^{k}
\end{array}\right.
$$

Once the inner loop in Eq. (23) is finished, the trust index in Eq. (26) is computed for updating both the design vector $\mathbf{V}^{k}$ and the TR radius vector $\boldsymbol{\delta}^{k}$ according to Eq. (27) for the next inner loop. Fig. 4 outlines the main sequential approximate strategy for the RBRDO. In the $k t h$ optimization cycle, calculate the actual penalty function $H_{p}\left(\mathbf{V}^{k}\right)$ first, whose computation process contains sample generation, actual model simulation and the gradient computation that are necessary for building the local explicit functions for the PMA functions in the subsequent step. Then solve the 
An efficient integrated framework for reliability-based robust design optimization of computation-intensive structures

sequential approximate RBRDO in Eq. (23) to obtain an optimal design vector $\mathbf{V}^{* k}$ for the $k t h$ optimization cycle. After that, the actual penalty function $H_{p}\left(\mathbf{V}^{* k}\right)$ can be computed. Further, update $\mathbf{V}^{k}$ and $\boldsymbol{\delta}^{k}$ according to $\rho^{k}$ if the stopping criterion is not satisfied. This paper sets a maximum iterative number as stopping criterion.

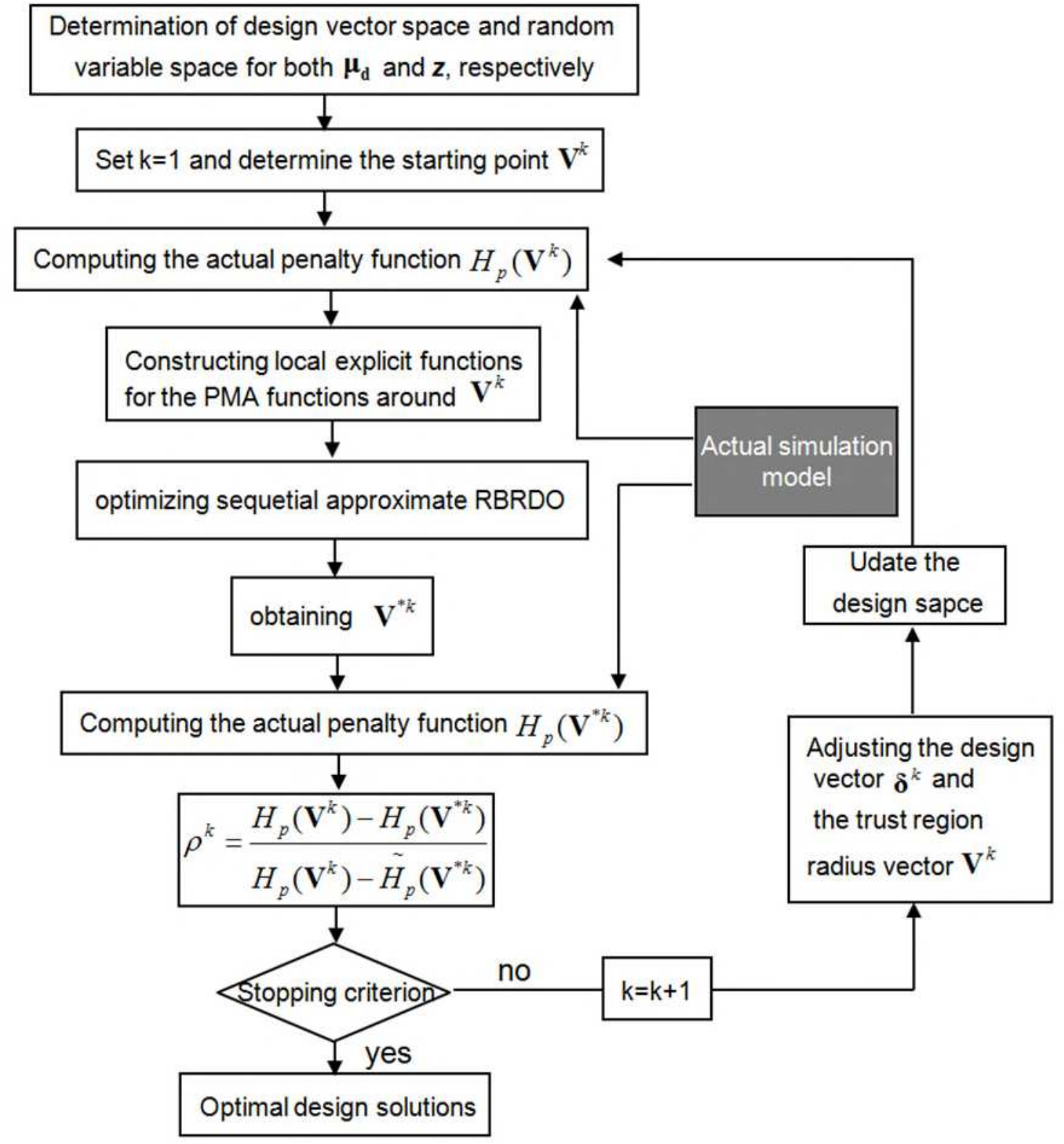

Fig. 4 The efficient sequential approximate RBRDO. 


\subsection{Approximate calculation of the actual penalty function}

To obtain the real value of the actual penalty function $H_{p}\left(\mathbf{V}^{* k}\right)$ in the outer loop, it necessitates the repeated computation of the actual simulation model to obtain the response of the performance function (i.e. $f_{p 1}, f_{p 2}$ and $\left.g_{j}\left(\mathbf{u}_{j}^{*} ; \mathbf{V}\right)\right)$. Hence, it's quite inefficient. Fig. 5 shows an efficient procedure to approximately compute the penalty function. Firstly, samples are generated according to Eq. (17) whose mean value is the specified design vector $\mathbf{V}$. Then compute the responses of the performance functions corresponding to the generated samples. After that, compute the gradients shown in Eq. (18) by the finite difference formulation. Through Eq. (15), the approximate MPPs and the corresponding PMA functions can be obtained. Lastly, the penalty function can be directly calculated in Eq. (12). It can be seen that the number of the actual model evaluation is limited, i.e. $\mathrm{N}+1$.

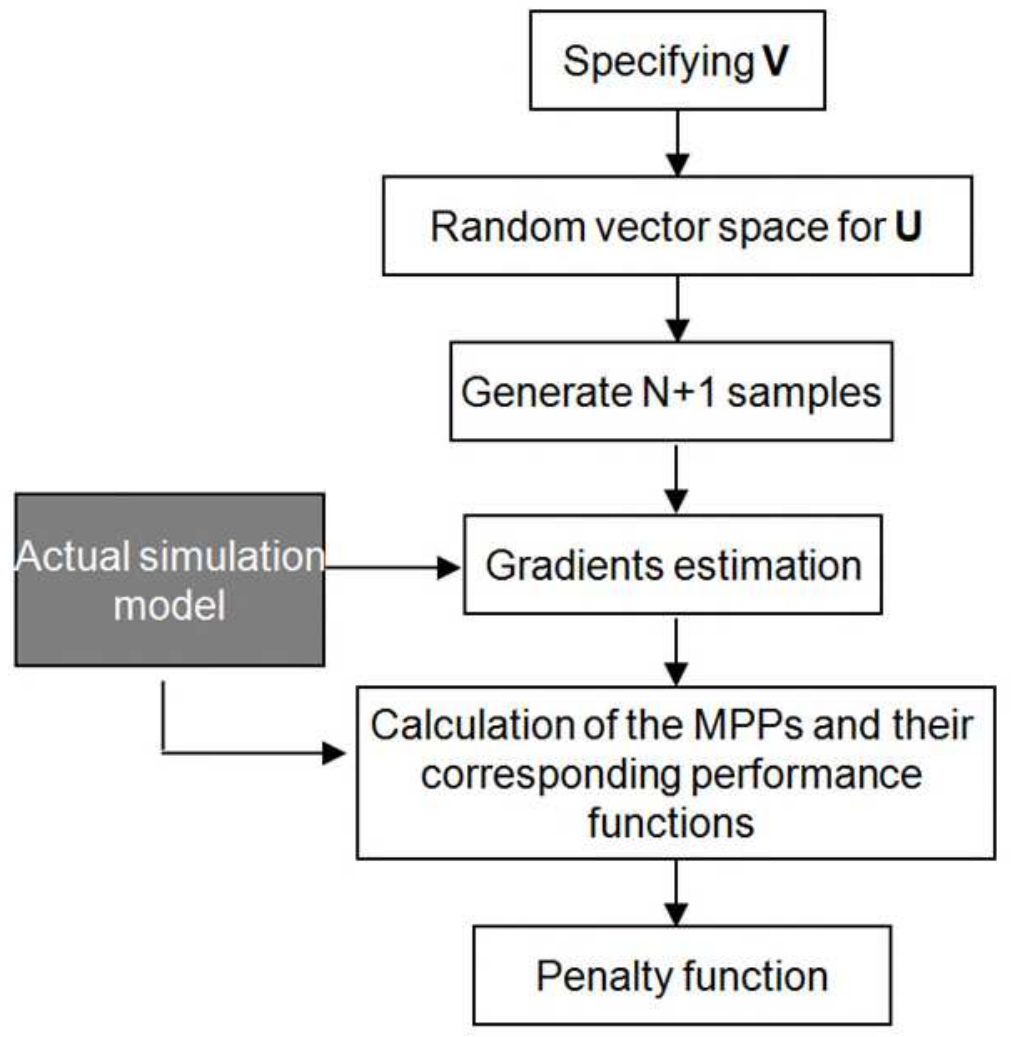


An efficient integrated framework for reliability-based robust design optimization of computation-intensive structures

Fig. 5 Computation of the actual penalty function at the specified design vector $\mathbf{V}$.

It is worth mentioning that the computed value of the penalty function is approximate rather than real in a rigorous sense. In spite of the above difference, this approximation is precise enough. Hence, it is still called "actual penalty function", distinguishing from the "approximate penalty function".

\section{Numerical examples}

\subsection{Test problem}
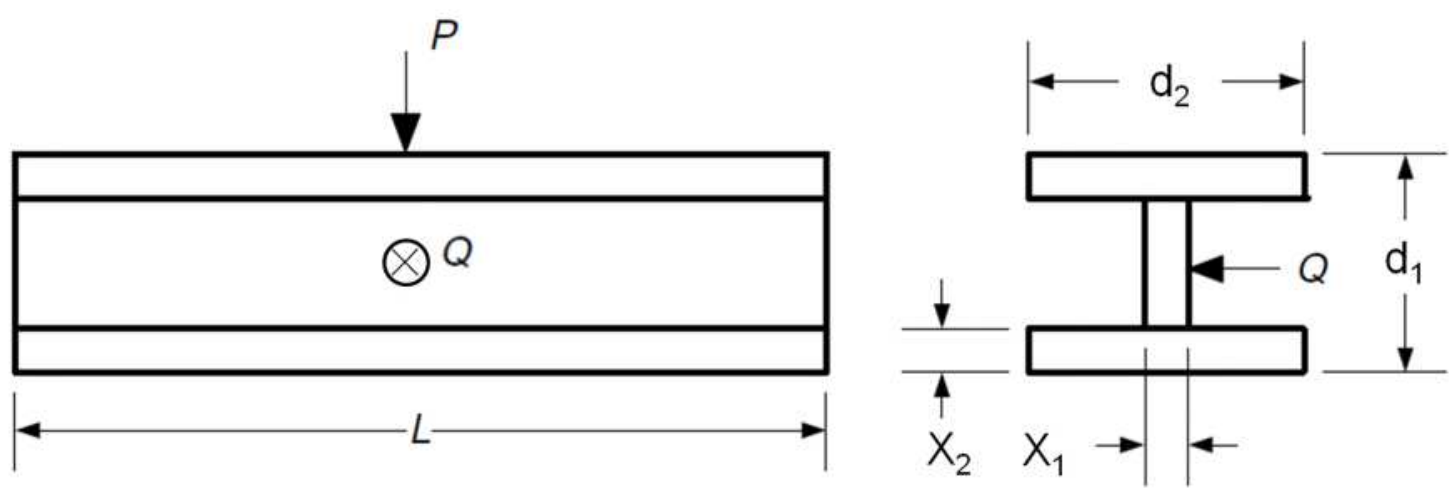

Fig. 6 A beam design problem.

A design problem of beam is shown in Fig. 6(Wang, 2003). The random vector is $\mathbf{U}=[\mathbf{d}, \mathbf{X}]^{T}=\left[d_{1}, d_{2}, X_{1}, X_{2}\right]^{T}$ and is supposed to be independent and normal. The design vector is the mean value of the former two components in $\mathbf{U}$, i.e. $\mathbf{V}=\left[\boldsymbol{\mu}_{\mathbf{d}}\right]^{T}=\left[\mu_{d 1}, \mu_{d 2}\right]^{T}$. The variation range and the standard deviation for $\mathbf{d}$ are specified as $\pm \mathbf{d}^{L} / 1000$ and $\mathbf{d}^{L} / 1000 / 3$, respectively. Table 1 shows the related parameters.

Table 1

Input parameters for the beam design problem. 
An efficient integrated framework for reliability-based robust design optimization of computation-intensive structures

\begin{tabular}{|c|c|c|c|c|}
\hline$d_{l}(\mathrm{~cm})$ & $\mu_{d 1}$ & $\pm d_{1}^{L} / 1000$ & & Normal \\
\hline \multirow[t]{2}{*}{$d_{2}(\mathrm{~cm})$} & $\mu_{d 2}$ & $\pm d_{2}^{L} / 1000$ & & Normal \\
\hline & & & $|\xi| / 3$ & \\
\hline$X_{I}(\mathrm{~cm})$ & 1 & \pm 0.1 & & Normal \\
\hline$X_{2}(\mathrm{~cm})$ & 2 & \pm 0.2 & & Normal \\
\hline $\begin{array}{l}\text { Young's Modulus } \\
E\left(\mathrm{kN} / \mathrm{cm}^{2}\right)\end{array}$ & $2 \times 10^{4}$ & ---- & ---- & ---- \\
\hline $\begin{array}{l}\text { Bending force } P \\
(\mathrm{kN})\end{array}$ & 600 & --- & ---- & --- \\
\hline $\begin{array}{l}\text { Bending force } Q \\
(\mathrm{kN})\end{array}$ & 50 & ---- & ---- & ---- \\
\hline Length $L(\mathrm{~cm})$ & 200 & --- & ---- & --- \\
\hline
\end{tabular}

The design problem is to minimize the vertical deflection on the premise of satisfying the cross-section area and stress constraints:

$$
\begin{gathered}
\min F(\mathbf{d}, \mathbf{X})=\frac{P L^{3}}{48 E I}=\frac{5000}{\frac{1}{12} X_{1}\left(d_{1}-2 X_{2}\right)^{3}+\frac{1}{6} d_{2} X_{2}^{3}+2 d_{2} X_{2}\left(\frac{d_{1}-X_{2}}{2}\right)^{2}} \\
G_{1}(\mathbf{d}, \mathbf{X})=300-2 d_{2} X_{2}-X_{1}\left(d_{1}-2 X_{2}\right) \leq 0 \\
G_{2}(\mathbf{d}, \mathbf{X})=8-\frac{180000 d_{1}}{X_{1}\left(d_{1}-2 X_{2}\right)^{3}+2 d_{2} X_{2}\left[4 X_{2}^{2}+3 d_{1}\left(d_{1}-2 X_{2}\right)\right]} \\
-\frac{15000 d_{2}}{\left(d_{1}-2 X_{2}\right) X_{1}^{3}+2 X_{2} d_{2}^{3}} \leq 0 \\
10 \mathrm{~cm} \leq \mu_{d 1} \leq 80 \mathrm{~cm} ; 10 \mathrm{~cm} \leq \mu_{d 2} \leq 50 \mathrm{~cm}
\end{gathered}
$$

where $\min F(\mathbf{d}, \mathbf{X})$ represents the minimization the vertical deflection, $G_{1}(\mathbf{d}, \mathbf{X})$ denotes that the cross-section area is no more than $300 \mathrm{~cm}^{2}, G_{2}(\mathbf{d}, \mathbf{X})$ is the stress constraint.

According to Eq. (12), Eq. (28) can be converted as: 


$$
\begin{array}{ll}
\min & H_{p}=W+\kappa \sum_{j=1}^{2} \max \left(0,-g_{j}\left(\mathbf{u}_{j}^{*} ; \mathbf{V}\right)\right) \\
& W=\alpha \mu_{f} / \phi+(1-\alpha) \Delta_{f} / \varphi \\
& \Delta_{f}=\max \left(\left|f_{0.00135}-\mu_{f}\right|,\left|f_{0.99865}-\mu_{f}\right|\right) \\
& f_{0.00135}=f\left(\mathbf{u}_{p 1}^{*} ; \mathbf{V}\right) \\
& f_{0.99865}=f\left(\mathbf{u}_{p 2}^{*} ; \mathbf{V}\right) \\
& \mathbf{V}^{L} \leq \mathbf{V} \leq \mathbf{V}^{R}
\end{array}
$$

where $\mathbf{V}^{L}=\left[\begin{array}{lll}10 & 10\end{array}\right]$ and $\mathbf{V}^{R}=\left[\begin{array}{lll}80 & 50\end{array}\right] . g_{1}\left(\mathbf{u}_{1}^{*} ; \mathbf{V}\right)$ and $g_{2}\left(\mathbf{u}_{2}^{*} ; \mathbf{V}\right)$ are equivalently corresponding to the first term and the second term in Eq. (30).

$$
\begin{aligned}
P\left\{G_{1}(\mathbf{U} ; \mathbf{V}) \leq 0\right\}= & P\left\{300-2 d_{2} X_{2}-X_{1}\left(d_{1}-2 X_{2}\right) \leq 0\right\} \leq \Phi(-3) \\
P\left\{G_{2}(\mathbf{U} ; \mathbf{V}) \leq 0\right\}= & P\left\{8-\frac{180000 d_{1}}{X_{1}\left(d_{1}-2 X_{2}\right)^{3}+2 d_{2} X_{2}\left[4 X_{2}^{2}+3 d_{1}\left(d_{1}-2 X_{2}\right)\right]}\right. \\
& \left.-\frac{15000 d_{2}}{\left(d_{1}-2 X_{2}\right) X_{1}^{3}+2 X_{2} d_{2}^{3}} \leq 0\right\} \leq \Phi(-3)
\end{aligned}
$$

The factors $\alpha, \phi, \varphi, \kappa, \beta^{f}, \beta^{j}, c$ and the maximum iterative number in optimization are set as $0.5,0.015,0.0029,100000,3,3,0.001$ and 20 respectively. Correspondingly, the maximum generations and the population size for PSO used in the general method are intended to be 100 and 30 .

\subsubsection{Convergence performance}

Next job is to test cases with different starting design points and different TR radii for analyzing the convergence ability of the proposed method. First of all, analyze four cases with different starting design points and the same TR radius. In Table 2, the TR radius of the four cases is specified as one 16th of the design space, i.e. $(4.375,2.5)$. The iterative optimization process is shown is Table 2 and Fig. 7. Curve1 Curve 4 are corresponding to the four cases shown in Table 2 . And the starting design points P1 P4 are $(10,10),(45,35),(60,45)$ and $(50,10)$, respectively. All the convergence curves of 
An efficient integrated framework for reliability-based robust design optimization of computation-intensive structures

these four cases converge to P0, i.e. $(80.00,50.00)$ which is also the computed result of the general method. Meanwhile, the iterative convergence speed is quite fast since the optimum results are achieved within no more than 8 iterative steps.

Table 2

Optimization results under with different starting design points.

$\begin{array}{ccccc} & 1 & & & \\ \\ \text { Iteration number }\end{array}$




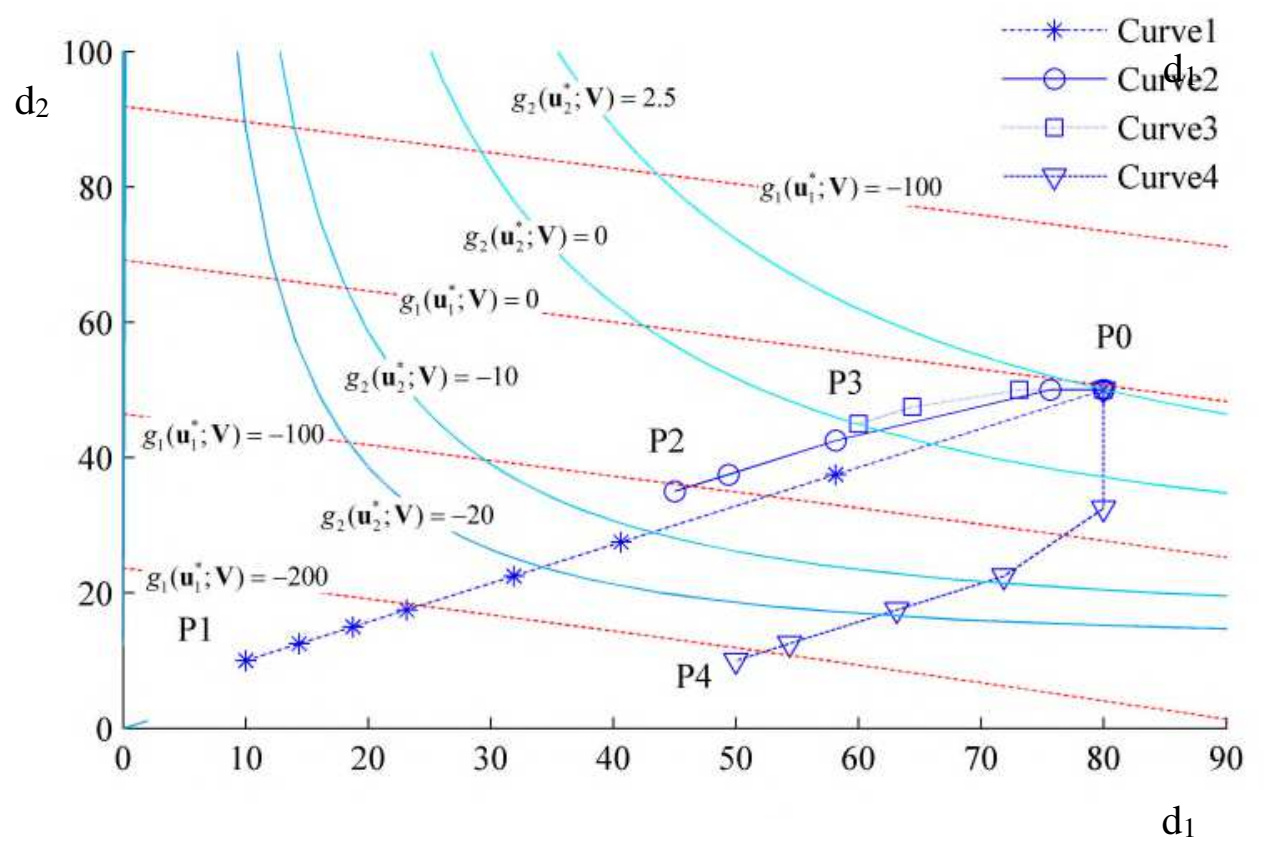

Fig. 7 Iterative optimization process with different starting design points.

In the following section, four cases with different starting design points and the same TR radius are also studied. The corresponding radii is one half, one 4th, one 8 th and one 16th of the design interval, respectively. The starting design point is specified as (10, 10). Table 3 lists the optimization results. Fig. 8 shows the iterative trajectory of the solutions. It can be found that the convergence trajectories of these four cases quickly converge to the same optimum P0, i.e. $(80.00,50.00)$, though the TR radii are different.

Table 3

Optimization results under with different TR radii.

\begin{tabular}{|c|c|c|c|c|}
\hline Cases & 1 & 2 & 3 & 4 \\
\hline 1 & $(10.00,10.00)$ & $(10.00,10.00)$ & $(10.00,10.00)$ & $(10.00,10.00)$ \\
\hline 2 & $(45.00,30.00)$ & $(27.50,20.00)$ & $(18.75,15.00)$ & $(14.38,12.50)$ \\
\hline 3 & $(80.00,50.00)$ & $(62.50,40.00)$ & $(27.50,20.00)$ & $(18.75,15.00)$ \\
\hline 4 & $(80.00,50.00)$ & $(80.00,50.00)$ & $(36.25,25.00)$ & $(23.13,17.50)$ \\
\hline
\end{tabular}


An efficient integrated framework for reliability-based robust design optimization of computation-intensive structures

\begin{tabular}{ccccc}
5 & $(80.00,50.00)$ & $(80.00,50.00)$ & $(45.00,30.00)$ & $(31.88,22.50)$ \\
6 & $(80.00,50.00)$ & $(80.00,50.00)$ & $(62.50,40.00)$ & $(40.63,27.50)$ \\
7 & $(80.00,50.00)$ & $(80.00,50.00)$ & $(80.00,50.00)$ & $(58.13,37.50)$ \\
$8 \sim 20$ & $(80.00,50.00)$ & $(80.00,50.00)$ & $(80.00,50.00)$ & $(80.00,50.00)$ \\
\hline
\end{tabular}

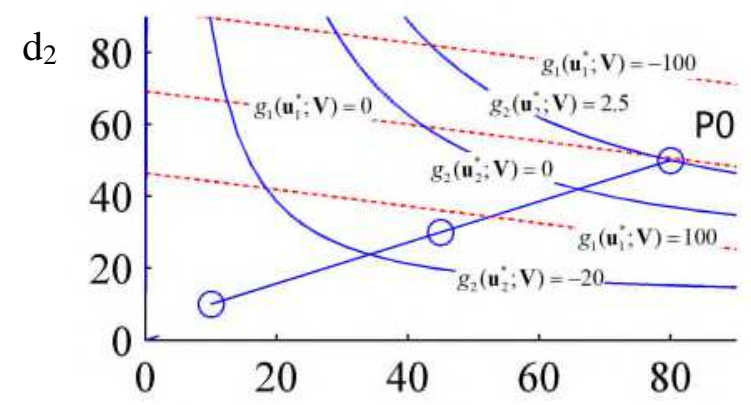

(a)

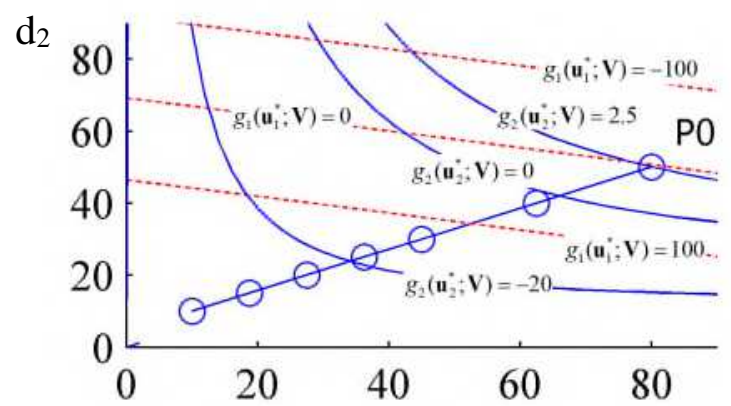

(c)

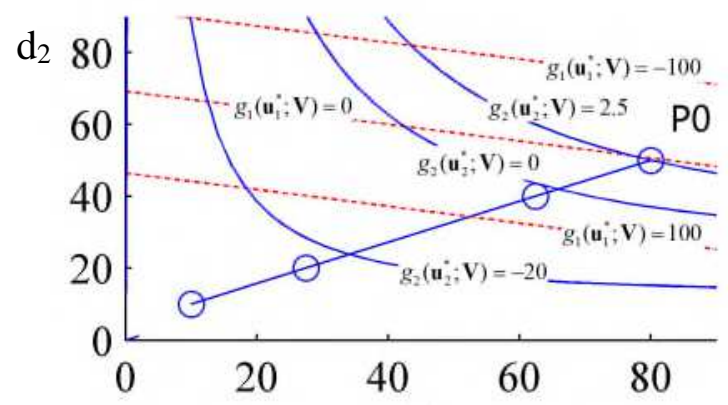

(b)

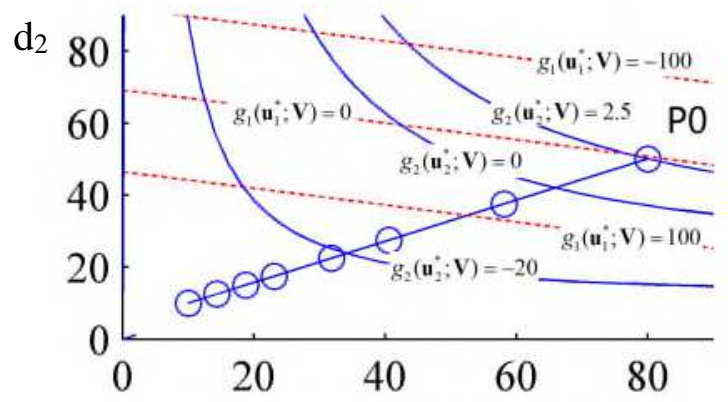

(d)

Fig. 8 Iterative trajectory with different TR radii.

In conclusion, the optimization results converge quickly with high speed, though the starting conditions are quite different. And it also shows that the convergence ability of the proposed method is robust.

\subsubsection{Optimization efficiency}

In this test, it is supposed that the objective function and the constraint functions in Eq. (37) are all time-consuming simulation models. The total computation times of all simulation models matters. For comparison, the general RBRDO showed in Fig. 2 is used for solving this test. Meanwhile, PSO as the optimization operator is applied for 
both the outer loop and the inner loop in the general RBRDO. And the objective function and the constraint functions are all computed by the actual simulation models. For the outer PSO, the total generated number of the particles is 3000 , since 30 particles with 100 iterations are carried out. For each particle, being a trial design vector, it needs to called the inner PSO fourth to calculate four PMA functions, and each particle also requires 3000 times of calculating the actual simulation models. Hence, the total computation times of the actual simulation model is $3000 \times 3000 \times 4=3.6 \times 10^{7}$. While using the proposed method, the actual simulation model should be carried out to evaluate the PMA functions when determining the actual penalty functions $H_{p}\left(\boldsymbol{\mu}_{\mathrm{d}}^{k}\right)$ and $H_{p}\left(\boldsymbol{\mu}_{\mathbf{d}}^{* k}\right)$, as showed in Fig. 4. In this example, when computing each PMA function at each iterative cycle, $5(=4+1)$ evaluations are needed for obtaining the MPP and 1 evaluation is needed for computing the value of PMA function of the MPP. Thus, only $24(=4 \times(5+1))$ evaluations are required for each iterative cycle. The total computation times of the PMA function is 480 for 20 iterative cycles. Hence, the ratio of the calculation scale for the above two methods is $3.6 \times 10^{7}$ to 480 . In a word, the proposed method is quite efficiency by greatly reducing the computation times of the actual simulation model, and it indicates that such advantage contributes to widespread engineering application.

\subsubsection{Analysis of the uncertainty level}

In the following paper, influences of the uncertainty level are examined. In the following paper, the RBRDO with different uncertainty levels of the design variables and random variables are carried out, respectively. 


\subsubsection{Analysis of the uncertainty level of the design variable}

As mentioned above, the design variables are treated as random ones in RBRDO. According to Ref. (Yu et al., 2013), the uncertainty levels of the design variables should be small enough, or the optimization accuracy can't be satisfied. In Table 4, cases with nine different quantitative levels for variation range of the design variables are examined. Meanwhile, the starting design point and the TR radius of the above cases are specified as $(10,10)$ and $(4.38,2.5)$, respectively. As showed in Table 4 , the optimum results are inaccurate, when the variation range $\xi$ is not small enough, i.e. $\xi= \pm \mathbf{d}^{L} / 10^{0}$. Additionally, the optimum results are not sensitive to the small variation range, even when the variation range is infinitesimal. The reason is that the components of gradients contributed by the design variables can be precisely computed by the finite difference formulation in Eq. (18), however small the variation range of the design variables is.

Table 4

Optimization results under different uncertainty levels of the design variables.

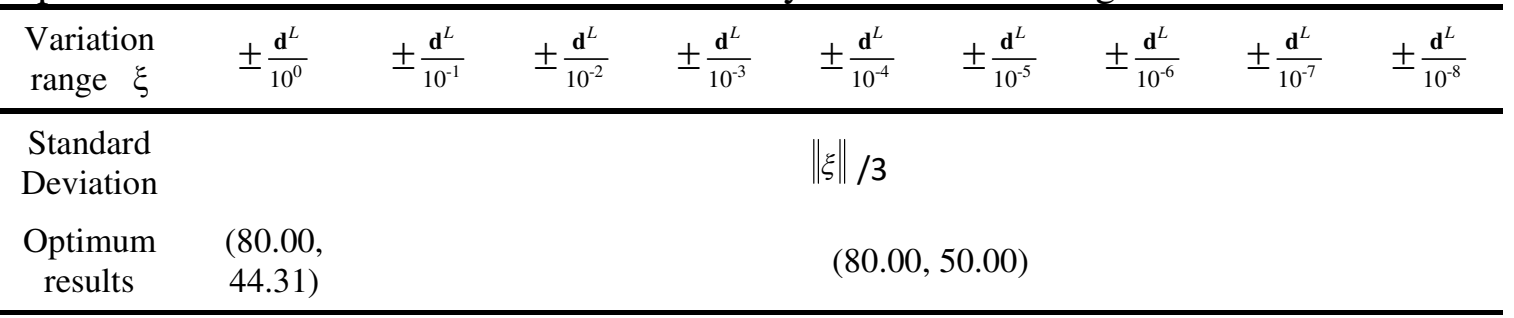

\subsubsection{Analysis of the uncertainty level of the random variable}

In Table 1, the ratio between the variation range of random variables and their mean values is $\pm 10 \%$. Besides, the other cases with the ratio being $\pm 20 \% \sim \pm 90 \%$ are also investigated. The starting design points and the trust radius vectors of the above cases are set as $(10,10)$ and $(4.375,2.5)$, respectively. Obviously, the larger the variation 
An efficient integrated framework for reliability-based robust design optimization of computation-intensive structures

range is, the smaller the possibility of the existing optimum that satisfies the constraints will be. In order to make sure that there are optimums for all of the cases, the constraints $g_{1}\left(\mathbf{u}_{1}^{*} ; \mathbf{V}\right)$ and $g_{2}\left(\mathbf{u}_{2}^{*} ; \mathbf{V}\right)$ in Eq. (29) are relaxed by trial and errors, and they are equivalently corresponding to the first term and the second term in Eq. (31), respectively.

$$
\begin{aligned}
P\left\{g_{1}\left(\mathbf{d}, \mathbf{X} ; \boldsymbol{\mu}_{\mathbf{d}}\right) \leq 0\right\} & =P\left\{300-2 d_{2} X_{2}-X_{1}\left(d_{1}-2 X_{2}\right) \leq 0\right\} \leq \Phi(-1.28) \approx 0.1 \\
P\left\{g_{2}\left(\mathbf{d}, \mathbf{X} ; \boldsymbol{\mu}_{\mathbf{d}}\right) \leq 0\right\} & =P\left\{8-\frac{180000 d_{1}}{X_{1}\left(d_{1}-2 X_{2}\right)^{3}+2 d_{2} X_{2}\left[4 X_{2}^{2}+3 d_{1}\left(d_{1}-2 X_{2}\right)\right]}\right. \\
& \left.-\frac{15000 d_{2}}{\left(d_{1}-2 X_{2}\right) X_{1}^{3}+2 X_{2} d_{2}^{3}} \leq 0\right\} \leq \Phi(-1.28) \approx 0.1
\end{aligned}
$$

For comparisons, Table 5 shows the optimization results computed by both the proposed method and the general method. Apparently, when the uncertainty level is less than $\pm 60 \%$, the deviations from the precise optimum are small and acceptable. However, with increases of the uncertainty level, the deviations are unreliable.

Table 5

Optimization results under different uncertainty levels of the random variables.

\begin{tabular}{cccccc}
\hline Ratio & Optimum results & $H_{p}$ & $\begin{array}{c}\text { Precise optimum } \\
\text { results }\end{array}$ & $\begin{array}{c}\text { Precise } \\
H_{p}\end{array}$ & $\begin{array}{c}\text { Deviations from the } \\
\text { precise optimum }\end{array}$ \\
\hline $\pm 10 \%$ & $(80.00,50.00)$ & 0.72 & $(80.00,50.00)$ & 0.60 & $(0 \%, 0 \%)$ \\
$\pm 20 \%$ & $(80.00,50.00)$ & 1.00 & $(80.00,50.00)$ & 0.71 & $(0 \%, 0 \%)$ \\
$\pm 30 \%$ & $(80.00,49.36)$ & 1.37 & $(80.00,49.35)$ & 0.81 & $(0 \%, 0 \%)$ \\
$\pm 40 \%$ & $(78.62,47.76)$ & 1.94 & $(80.00,47.44)$ & 0.93 & $(1.72 \%, 0.67 \%)$ \\
$\pm 50 \%$ & $(74.53,46.88)$ & 2.57 & $(80.00,45.66)$ & 2.56 & $(0.06 \%, 0.03 \%)$ \\
\hline
\end{tabular}


An efficient integrated framework for reliability-based robust design optimization of computation-intensive structures

\begin{tabular}{lccccc}
\hline $\pm 60 \%$ & $(80.00,43.99)$ & 3.51 & $(77.75,44.40)$ & 1.55 & $(2.9 \%, 0.91 \%)$ \\
$\pm 70 \%$ & $(80.00,42.42)$ & 38454 & $(58.83 \%, 33.97 \%)$ & 11.71 & $(35.98 \%, 24.87 \%)$ \\
$\pm 80 \%$ & $(80.00,40.95)$ & 124900 & $(50.15 \%, 45.50 \%)$ & 4.38 & $(59.52 \%, 10.02 \%)$ \\
$\pm 90 \%$ & $(80.00,39.55)$ & 222727 & $(55.03 \%, 33.61 \%)$ & 4.88 & $(45.37 \%, 17.68 \%)$ \\
\hline
\end{tabular}

\subsection{Application 1}

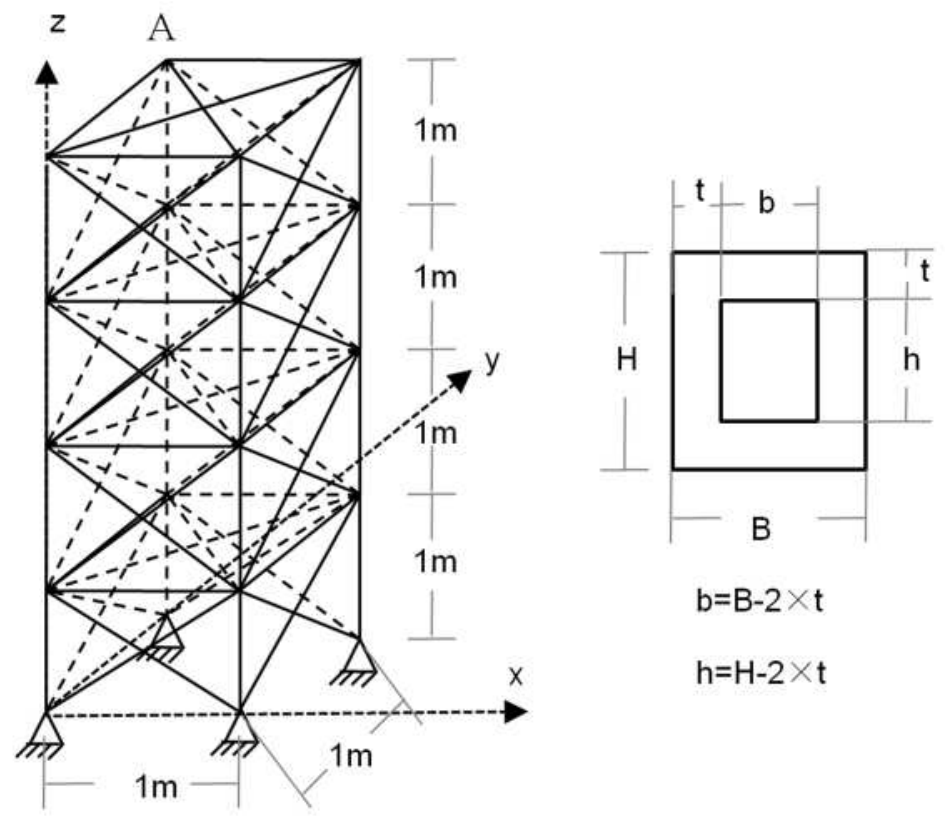

Fig. 9 A 72-bar truss.

The robust optimization design of a 72-bar truss is considered in Fig. 9. In this structure, a concentrated load being $50 \mathrm{kN}$ is acting on the point $\mathrm{A}$ along the $\mathrm{x}$ coordinate. The maximum allowable stress and displacement for all bars should not exceed $150 \mathrm{MPa}$ and $0.003 \mathrm{~m}$, respectively. Table 6 shows the related statistical parameters. $H, B, t$ are the main sizes of the cross-section of the hollow box beam. $\rho$ is the density of material. E is the Young's Modulus of material. The random vector is $\mathbf{U}=[H, B, t, E, \rho, P]$. All variables in $\mathbf{U}$ are taken as normal and statistically independent. The mean values of the former three components in $\mathbf{U}$ is combined as the 
An efficient integrated framework for reliability-based robust design optimization of computation-intensive structures

design vector, i.e. $\mathbf{V}=\left[\boldsymbol{\mu}_{\mathbf{d}}\right]^{T}=\left[\mu_{H}, \mu_{B}, \mu_{t}\right]$.The variation ranges and the standard deviation for $\mathbf{d}$ are specified as $\pm \mathbf{d}^{L} / 1000$ and $\mathbf{d}^{L} / 1000 / 3$, respectively.

Table 6

Input parameters for the 72-bar truss design problem.

\begin{tabular}{ccccc}
\hline Name & Mean Value & $\begin{array}{c}\text { Range of the } \\
\text { variation }(\xi)\end{array}$ & $\begin{array}{c}\text { Standard } \\
\text { Deviation }\end{array}$ & Distribution Type \\
\hline$H(\mathrm{~m})$ & $\mu_{H}$ & $\pm H^{L} / 1000$ & & Normal \\
$B(\mathrm{~m})$ & $\mu_{B}$ & $\pm B^{L} / 1000$ & Normal \\
$t(\mathrm{~m})$ & $\mu_{h}$ & $\pm t^{L} / 1000$ & Normal \\
$\begin{array}{c}\text { Young's Modulus } \\
E(\mathrm{MPa})\end{array}$ & $2.1 \times 10^{5}$ & $\pm 6.3 \times 10^{4}$ & $\mid \xi / 3$ & Normal \\
$\begin{array}{c}\text { Density } \rho \\
\left(\mathrm{kg} / \mathrm{m}^{3}\right)\end{array}$ & 7860 & \pm 2358 & & Normal \\
$\begin{array}{c}\text { The concentrated } \\
\text { force } P(\mathrm{kN})\end{array}$ & 100 & \pm 30 & Normal \\
\hline
\end{tabular}

The design problem is the minimization of the structural weight with the limitation of the stress constraint, displacement constraint and fundamental frequency constraint. That is,

$$
\begin{aligned}
& \min F(\mathbf{d}, \mathbf{X})=\left(32 \times 1+40 \times 1 \times 2^{0.5}\right) \times[H \times B-b \times h] \times \rho \\
& G_{1}(\mathbf{d}, \mathbf{X})=150-\sigma_{\max }(\mathbf{d}, \mathbf{X}) \leq 0 \\
& G_{2}(\mathbf{d}, \mathbf{X})=0.003-u_{\max }(\mathbf{d}, \mathbf{X}) \leq 0 \\
& G_{3}(\mathbf{d}, \mathbf{X})=0.1-B / H \leq 0 \\
& G_{4}(\mathbf{d}, \mathbf{X})=B / H-0.5 \leq 0 \\
& G_{5}(\mathbf{d}, \mathbf{X})=0.1-b / h \leq 0 \\
& G_{6}(\mathbf{d}, \mathbf{X})=b / h-0.5 \leq 0 \\
& 15 \mathrm{~mm} \leq \mu_{H} \leq 45 \mathrm{~mm} \\
& 15 \mathrm{~mm} \leq \mu_{B} \leq 45 \mathrm{~mm} \\
& 3 \mathrm{~mm} \leq \mu_{t} \leq 10 \mathrm{~mm}
\end{aligned}
$$


where $\min F(\mathbf{d}, \mathbf{X})$ represents the minimization of the total weight, $G_{1}(\mathbf{d}, \mathbf{X})$ denotes the stress constraint, $G_{2}(\mathbf{d}, \mathbf{X})$ is the displacement constraint. $G_{3}(\mathbf{d}, \mathbf{X}) \sim G_{6}(\mathbf{d}, \mathbf{X})$ are the geometrical constraints.

According to Eq. (12), Eq. (32) can be converted as:

$$
\begin{array}{ll}
\min & H_{p}=W+\kappa \sum_{j=1}^{6} \max \left(0,-g_{j}\left(\mathbf{u}_{j}^{*} ; \mathbf{V}\right)\right) \\
& W=\alpha \mu_{f} / \phi+(1-\alpha) \Delta_{f} / \varphi \\
& \Delta_{f}=\max \left(\left|f_{0.00135}-\mu_{f}\right|,\left|f_{0.99865}-\mu_{f}\right|\right) \\
& f_{0.00135}=f\left(\mathbf{u}_{p 1}^{*} ; \mathbf{V}\right) \\
& f_{0.99865}=f\left(\mathbf{u}_{p 2}^{*} ; \mathbf{V}\right) \\
& \mathbf{V}^{L} \leq \mathbf{V} \leq \mathbf{V}^{R}
\end{array}
$$

where $\mathbf{V}^{L}=\left[\begin{array}{lll}15 & 15 & 3\end{array}\right]$ and $\mathbf{V}^{R}=\left[\begin{array}{lll}45 & 45 & 10\end{array}\right] . \quad g_{j}\left(\mathbf{u}_{j}^{*} ; \mathbf{V}\right) \quad(j=1 \sim 0)$ are equivalently corresponding to $P\left\{g_{j}(\mathbf{U} ; \mathbf{V}) \leq 0\right\}(j=1 \sim 0)$ in Eq. (43).

$$
\begin{aligned}
& P\left\{g_{1}(\mathbf{U} ; \mathbf{V}) \leq 0\right\}=P\left\{150-\sigma_{\max }(\mathbf{U} ; \mathbf{V}) \leq 0\right\} \leq \Phi(-1.28) \approx 0.1 \\
& P\left\{g_{2}(\mathbf{U} ; \mathbf{V}) \leq 0\right\}=P\left\{0.003-u_{\max }(\mathbf{U} ; \mathbf{V}) \leq 0\right\} \leq \Phi(-1.28) \approx 0.1 \\
& P\left\{g_{3}(\mathbf{U} ; \mathbf{V}) \leq 0\right\}=P\{0.1-B / H \leq 0\} \leq \Phi(-1.28) \approx 0.1 \\
& P\left\{g_{4}(\mathbf{U} ; \mathbf{V}) \leq 0\right\}=P\{B / H-0.5 \leq 0\} \leq \Phi(-1.28) \approx 0.1 \\
& P\left\{g_{5}(\mathbf{U} ; \mathbf{V}) \leq 0\right\}=P\{0.1-b / h \leq 0\} \leq \Phi(-1.28) \approx 0.1 \\
& P\left\{g_{6}(\mathbf{U} ; \mathbf{V}) \leq 0\right\}=P\{b / h-0.5 \leq 0\} \leq \Phi(-1.28) \approx 0.1
\end{aligned}
$$

The structure is modeled by using the FEM, and the solution of the stress and the vertical displacement constraints in Eq. (32) must call FEM. Here the computation times of the FEM is concerned. The factors $\alpha, \phi, \varphi, \kappa, \beta^{f}, \beta^{j}$ and $c$ are specified as $0.5,1,1,10^{5}, 3,1.28$ and 0.001 , respectively. Maximum generations and the population size for PSO are specified as 100 and 30. The starting design point is specified as (15, 
An efficient integrated framework for reliability-based robust design optimization of computation-intensive structures

$15,5)$ and the maximum iterative number is specified as 20 for the sequential optimization cycle.

Table 7 lists the optimization results, and Fig. 10 shows the iterative optimization history. Obviously, the convergence speed of the proposed method is quite high. It can be found that the optimal design solution becomes stable after 11 iterations. At iteration number 18 , the penalty functions converge to 239.64 . At this optimal design, the values of $\mu_{f}, f_{0.00135}$ and $f_{0.99865}$ are $368.68,258.07$ and 479.28 , respectively, and the constraints are satisfied with both $g_{1}\left(\mathbf{u}_{1}^{*} ; \mathbf{V}\right)$ and $g_{2}\left(\mathbf{u}_{2}^{*} ; \mathbf{V}\right)$ greater than 0 . For each iterative step, it needs to call FEM $16(=2 \times 2 \times(3+1))$ times. The total number of the FEM evaluations is $320(=16 \times 20)$ for 20 iterative steps. Hence, the present method is proved to be efficient.

\section{Table 7}

Optimization results of the 72-bar truss (m).

\begin{tabular}{cccccccc}
\hline $\begin{array}{c}\text { Iteration } \\
\text { number } \\
k\end{array}$ & $\begin{array}{c}\text { Design vector } \\
\boldsymbol{\mu}_{\mathrm{d}}^{k}\end{array}$ & $\begin{array}{c}\text { Penalty } \\
\text { function } \\
H_{p}\left(\boldsymbol{\mu}_{\mathrm{d}}^{k}\right)\end{array}$ & $\mu_{f}$ & $f_{0.00135}$ & $f_{0.99865}$ & $g_{1}\left(\mathbf{u}_{1}^{*} ; \mathbf{V}\right)$ & $g_{2}\left(\mathbf{u}_{2}^{*} ; \mathbf{V}\right)$ \\
\hline 1 & $(15,15,5)$ & $2.48 \times 10^{7}$ & 139.23 & 97.46 & 181.00 & -247.18 & $9.90 \times 10^{-4}$ \\
2 & $(16.5,16.5,5.5)$ & $1.79 \times 10^{7}$ & 168.47 & 117.93 & 219.01 & -178.25 & 0.0013 \\
3 & $(19.5,19.5,6.5)$ & $8.60 \times 10^{6}$ & 235.30 & 164.71 & 305.89 & -85.02 & 0.0018 \\
4 & $(25.35,20.52,8.47)$ & $1.36 \times 10^{6}$ & 338.67 & 237.07 & 440.27 & -13.28 & 0.0022 \\
5 & $(31.35,19.30,6.90)$ & $4.86 \times 10^{5}$ & 357.32 & 250.12 & 464.51 & -4.76 & 0.0022 \\
$6 \sim 7$ & $(36.42,18.29,6.84)$ & $2.25 \times 10^{5}$ & 363.21 & 254.25 & 472.17 & -2.25 & 0.0022 \\
8 & $(36.79,17.91,6.71)$ & $1.68 \times 10^{5}$ & 364.60 & 255.22 & 473.98 & -1.67 & 0.0022 \\
9 & $(36.41,17.54,6.59)$ & 4695.4 & 368.56 & 257.99 & 479.12 & -0.0446 & 0.0022 \\
$10 \sim 20$ & $(36.36,17.49,6.57)$ & 239.64 & 368.68 & 258.07 & 479.28 & 0.0050 & 0.0022
\end{tabular}




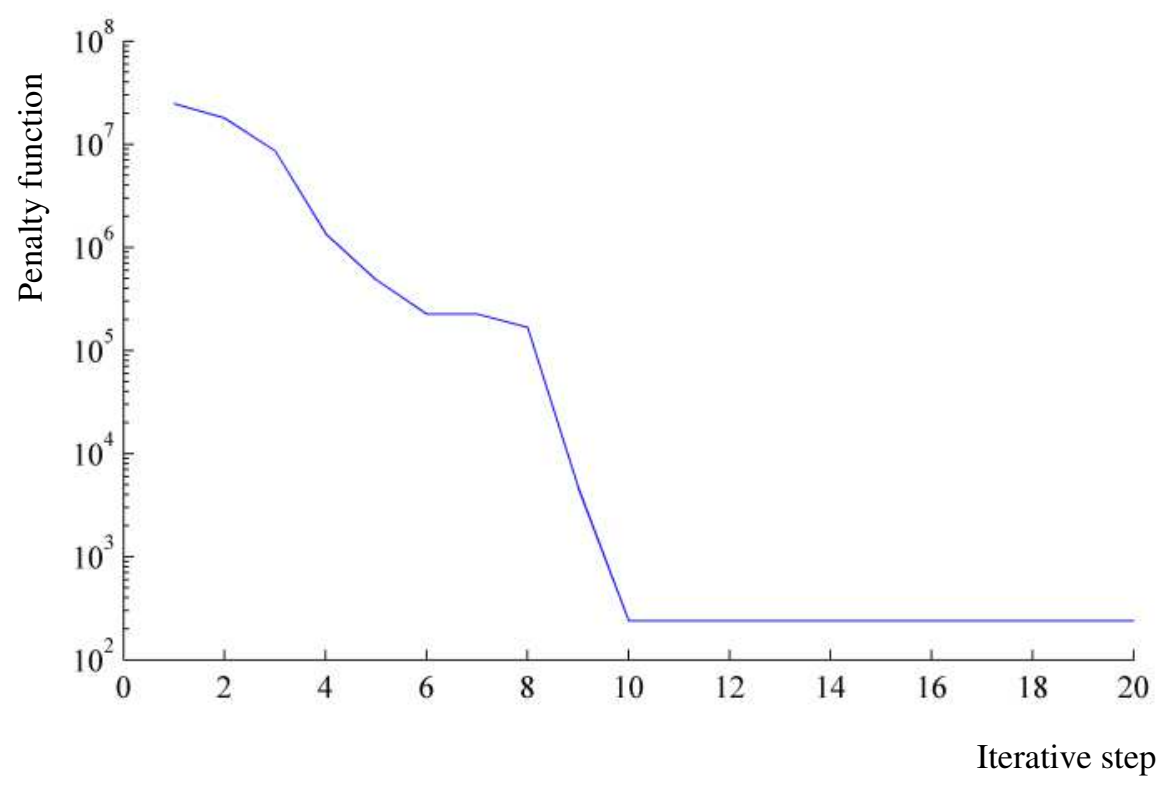

Fig. 10 Iteration history of the 72-bar truss.

\subsection{Application 2}

This example considers an optimization of the piezoresistive pressure sensor. As for this sensor shown in Fig. 11, a silicon membrane is bonded on a glass support. And a piezoresistor (PZT) is pasted on the silicon membrane for converting the mechanical stress into a voltage. Then the pressure acted on the silicon membrane can be obtained by measuring the voltage of PZT.

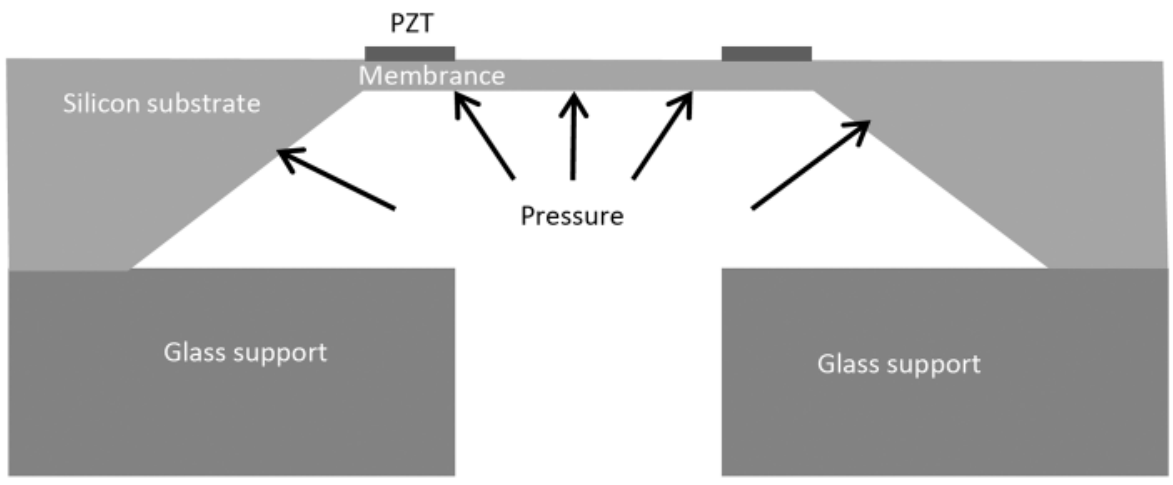


Fig. 11. Schema of pressure sensor.

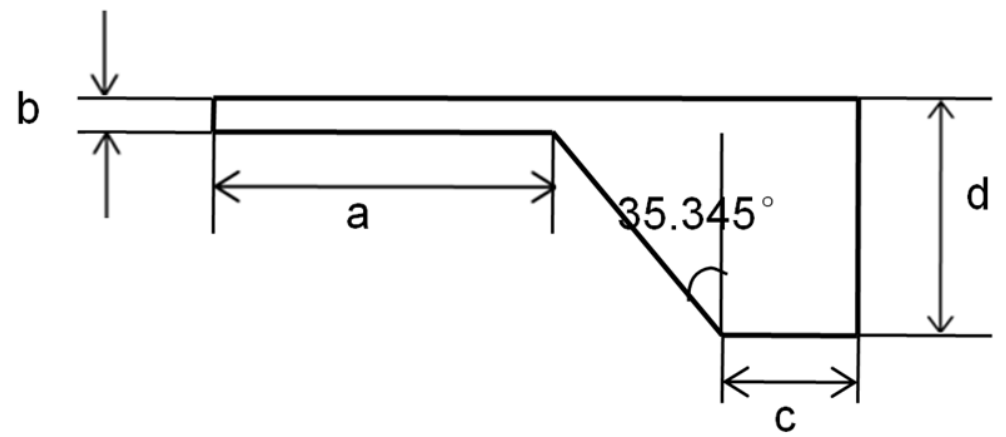

Fig.12 The main sizes of pressure sensor.

Fig. 12 shows the main sizes design of the pressure sensor. The random factor considered is $\mathbf{U}=[a, b, c, d, E, N u, P]$. All variables in $\mathbf{U}$ are normal and independent. $E$ is Young modulus. $\mathrm{Nu}$ is Poisson's ratio. $\mathrm{P}$ is pressure. The design vector is the mean value of the former four components in $\mathbf{U}$, i.e. $\mathbf{V}=\left[\boldsymbol{\mu}_{\mathbf{d}}\right]^{T}=\left[\mu_{a}, \mu_{b}, \mu_{c}, \mu_{d}\right]$. The variation ranges and the standard deviation for $\mathbf{d}$ are specified as $\pm \mathbf{d}^{L} / 1000$ and $\mathbf{d}^{L} / 1000 / 3$, respectively. Concerned parameters are shown in Table 8 .

Table 8

Input parameters for the pressure sensor design problem.

\begin{tabular}{ccccc}
\hline Name & Mean Value & $\begin{array}{c}\text { Range of the } \\
\text { variation }(\xi)\end{array}$ & $\begin{array}{c}\text { Standard } \\
\text { Deviation }\end{array}$ & Distribution Type \\
\hline$a(\mathrm{~m})$ & $\mu_{a}$ & $\pm a^{L} / 1000$ & & Normal \\
$b(\mathrm{~m})$ & $\mu_{b}$ & $\pm b^{L} / 1000$ & Normal \\
$c(\mathrm{~m})$ & $\mu_{c}$ & $\pm c^{L} / 1000$ & Normal \\
$d(\mathrm{~m})$ & $\mu_{d}$ & $\pm d^{L} / 1000$ & $\mid \xi / 3$ & Normal \\
$\begin{array}{c}\text { Density } \\
\rho\left(\mathrm{kg} / \mathrm{m}^{3}\right)\end{array}$ & $2.34 \times 10^{3}$ & \pm 702 & Normal \\
$\begin{array}{c}\text { Young's Modulus } \\
E(\mathrm{MPa})\end{array}$ & $1.689 \times 10^{11}$ & $\pm 5.067 \times 10^{10}$ & & Normal \\
\hline
\end{tabular}


An efficient integrated framework for reliability-based robust design optimization of computation-intensive structures

\begin{tabular}{cccc}
\hline $\begin{array}{c}\text { Poisson's ratio Nu } \\
\begin{array}{c}\text { The pressure } P \\
\text { (Mpa) }\end{array}\end{array}$ & 0.0624 & \pm 0.01872 & Normal \\
\hline
\end{tabular}

The design problem is to minimize the stress of the pressure sensor and simultaneously satisfies the displacement and fundamental frequency constraints under given loads, namely:

$$
\begin{aligned}
& \min \sigma_{\max }\left(\mathbf{d}, \mathbf{X} ; \boldsymbol{\mu}_{\mathbf{d}}\right) \\
& g_{1}\left(\mathbf{d}, \mathbf{X} ; \boldsymbol{\mu}_{\mathbf{d}}\right)=0.05-d_{\text {max }}\left(\mathbf{d}, \mathbf{X} ; \boldsymbol{\mu}_{\mathbf{d}}\right) \leq 0 \\
& g_{2}\left(\mathbf{d}, \mathbf{X} ; \boldsymbol{\mu}_{\mathbf{d}}\right)=12000-\omega\left(\mathbf{d}, \mathbf{X} ; \boldsymbol{\mu}_{\mathbf{d}}\right) \leq 0 \\
& 0.5 \times 10^{-3} \mathrm{~m} \leq \mu_{a} \leq 2 \times 10^{-3} \mathrm{~m} ; 0.01 \times 10^{-3} \mathrm{~m} \leq \mu_{b} \leq 0.05 \times 10^{-3} \mathrm{~m} \\
& 0.1 \times 10^{-3} \mathrm{~m} \leq \mu_{c} \leq 1 \times 10^{-3} \mathrm{~m} ; 0.3 \times 10^{-3} \mathrm{~m} \leq \mu_{d} \leq 0.8 \times 10^{-3} \mathrm{~m}
\end{aligned}
$$

where $\min \sigma_{\max }\left(\mathbf{d}, \mathbf{X} ; \boldsymbol{\mu}_{\mathbf{d}}\right)$ denotes the minimization of the maximum stress, $g_{1}\left(\mathbf{d}, \mathbf{X} ; \boldsymbol{\mu}_{\mathbf{d}}\right)$ is displacement constraint, $g_{2}\left(\mathbf{d}, \mathbf{X} ; \boldsymbol{\mu}_{\mathbf{d}}\right)$ is fundamental frequency constraint.

According to Eq. (12), Eq. (35) can be converted as:

$$
\begin{array}{ll}
\min & H_{p}=W+\kappa \sum_{j=1}^{2} \max \left(0,-g_{j}\left(\mathbf{u}_{j}^{*} ; \mathbf{V}\right)\right) \\
& W=\alpha \mu_{f} / \phi+(1-\alpha) \Delta_{f} / \varphi \\
& \Delta_{f}=\max \left(\left|f_{0.00135}-\mu_{f}\right|,\left|f_{0.99865}-\mu_{f}\right|\right) \\
& f_{0.00135}=f\left(\mathbf{u}_{p 1}^{*} ; \mathbf{V}\right) \\
& f_{0.99865}=f\left(\mathbf{u}_{p 2}^{*} ; \mathbf{V}\right) \\
& \mathbf{V}^{L} \leq \mathbf{V} \leq \mathbf{V}^{R}
\end{array}
$$

where $\mathbf{V}^{L}=\left[0.5 \times 10^{-3} 0.01 \times 10^{-3} 0.1 \times 10^{-3} 0.3 \times 10^{-3}\right]$ and $\mathbf{V}^{R}=\left[2 \times 10^{-3} 0.05 \times 10^{-3}\right.$ $\left.1 \times 10^{-3} 0.8 \times 10^{-3}\right] \quad . \quad g_{j}\left(\mathbf{u}_{j}^{*} ; \mathbf{V}\right) \quad(j=1 \sim 2) \quad$ are equivalently corresponding to $P\left\{g_{j}(\mathbf{U} ; \mathbf{V}) \leq 0\right\}$ in Eq. (36). 


$$
\begin{aligned}
& P\left\{g_{1}(\mathbf{U} ; \mathbf{V}) \leq 0\right\}=P\left\{0.05-d_{\text {max }}\left(\mathbf{d}, \mathbf{X} ; \boldsymbol{\mu}_{\mathbf{d}}\right) \leq 0\right\} \leq \Phi(-3) \\
& P\left\{g_{2}(\mathbf{U} ; \mathbf{V}) \leq 0\right\}=P\left\{12000-\omega\left(\mathbf{d}, \mathbf{X} ; \boldsymbol{\mu}_{\mathbf{d}}\right) \leq 0\right\} \leq \Phi(-3)
\end{aligned}
$$

The device is also modeled by using an axisymmetric finite element model (FEM) and its maximum stress, the maximum displacement and the fundamental frequency can be simultaneously obtained. Hence, the evaluation number of the FEM is concerned.

The factors $\alpha, \phi, \varphi, \kappa, \beta^{f}, \beta^{j}$ and $c$ are specified as $0.5,1,1,10^{6}, 3,3$ and 0.001, respectively. Maximum generations and the population size for PSO are specified as 100 and 30 . The maximum iterative number is specified as 16 for the sequential optimization cycle. And the starting design point is specified as $\left(0.5 \times 10^{-3}\right.$, $\left.0.01 \times 10^{-3}, 0.1 \times 10^{-3}, 0.3 \times 10^{-3}\right)$. Table 9 lists the optimization results. Fig. 13 shows the iterative optimization history. Seen from Table 9 and Fig. 13, a stable optimal solution is achieved after the 7th iteration. At this optimal solution, the values of $\mu_{f}$ , $f_{\mathrm{p} 1}$ and $f_{\mathrm{p} 2}$ are $149.98,104.98$ and 195.02 , respectively, and the constraints are satisfied with both $g_{1}\left(\mathbf{z}_{1}^{* k} ; \boldsymbol{\mu}_{\mathbf{d}}^{k}\right)$ and $g_{2}\left(\mathbf{z}_{1}^{* k} ; \boldsymbol{\mu}_{\mathbf{d}}^{k}\right)$ greater than 0 . For each iterative step, it needs to call FEM $30(=2 \times 3 \times(4+1))$ times. And the total computation times of calling the FEM is $480(=16 \times 30)$ for 16 iterative steps. Obviously, the presented method is quite efficient, since it converges fast.

Table 9

Optimization results of the pressure sensor $\left(\mathrm{m}^{-3}\right)$.

\begin{tabular}{cccccccc}
\hline $\begin{array}{c}\text { Iteration } \\
\text { number } \\
k\end{array}$ & $\begin{array}{c}\text { Design vector } \\
\boldsymbol{\mu}_{\mathrm{d}}^{k}\end{array}$ & $\begin{array}{c}\text { Penalty } \\
\text { function } \\
H_{p}\left(\boldsymbol{\mu}_{\mathrm{d}}^{k}\right)\end{array}$ & $\mu_{f}$ & $f_{0.00135}$ & $f_{0.99865}$ & $g_{1}\left(\mathbf{u}_{1}^{*} ; \mathbf{V}\right)$ & $g_{2}\left(\mathbf{u}_{2}^{*} ; \mathbf{V}\right)$ \\
\hline 1 & $(0.5,0.01,0.1,0.3)$ & $1.87 \times 10^{10}$ & 88.14 & 61.70 & 114.61 & 0.044 & $-1.87 \times 10^{4}$ \\
2 & $\begin{array}{c}(0.54955,0.0105,0 \\
.1,0.3)\end{array}$ & $1.47 \times 10^{10}$ & 96.64 & 67.64 & 125.66 & 0.043 & $-1.47 \times 10^{4}$
\end{tabular}


An efficient integrated framework for reliability-based robust design optimization of computation-intensive structures

\begin{tabular}{|c|c|c|c|c|c|c|c|}
\hline 3 & $\begin{array}{c}(0.59885,0.0105,0 \\
.1,0.3)\end{array}$ & $1.06 \times 10^{10}$ & 114.90 & 80.42 & 149.40 & 0.040 & $-1.06 \times 10^{4}$ \\
\hline 4 & $\begin{array}{c}(0.69755,0.0125,0 \\
.1,0.36)\end{array}$ & $7.77 \times 10^{9}$ & 109.96 & 76.97 & 142.98 & 0.039 & -7772.62 \\
\hline 5 & $\begin{array}{c}(0.79555,0.0145,0 \\
.1,0.3)\end{array}$ & $5.61 \times 10^{9}$ & 106.66 & 74.24 & 137.91 & 0.038 & -5612.81 \\
\hline 6 & $\begin{array}{c}(0.8935,0.0125,0 \\
14,0.3)\end{array}$ & $1.13 \times 10^{8}$ & 180.37 & 126.25 & 234.53 & 0.021 & -112.79 \\
\hline $7 \sim 13$ & $\begin{array}{c}(1.090,0.0165,0.1 \\
4,0.3)\end{array}$ & 100.14 & 154.03 & 107.81 & 200.28 & 0.022 & 1282.44 \\
\hline $14 \sim 16$ & $\begin{array}{c}(1.084,0.0166,0.1 \\
3875,0.3)\end{array}$ & 97.51 & 149.98 & 104.98 & 195.02 & 0.023 & 1079.736 \\
\hline
\end{tabular}

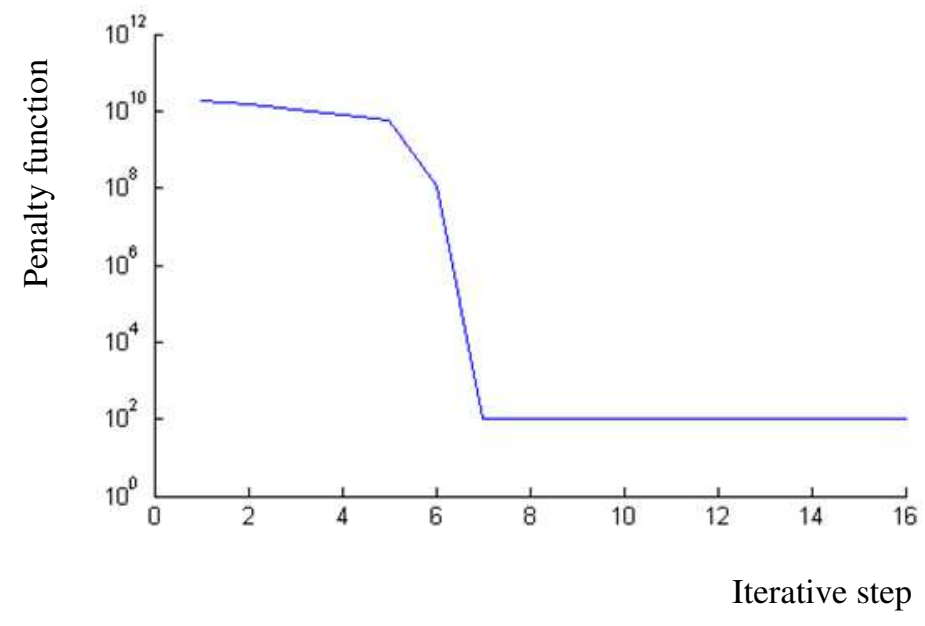

Fig. 13 Iteration history of the pressure sensor design.

\section{Conclusion}

Aiming at the uncertain optimization design of the computation-intensive structures, the paper proposes an efficient integrated framework of RBRDO. Through PMA, the RBRDO problem is transformed into an improved percentile form. In the improved formulation above, a series of new approximation methods are proposed to accelerate the computation. Firstly, PMA functions are estimated by an efficient approximation method. Secondly, the improved form above to RBRDO problem is 
changed as a sequential approximate RBRDO, in which the original objective function and constraints are replaced by approximate explicit form. And the explicit form is only in relation to the design variables. Then the TR method is used to manage sequential approximate sub-optimization problems and thereby to ensure the optimization convergence. In this way, the above approximation methods can greatly ease the frequent update of the design vector in the outer loop which in turn decreases the number of reconstructing the surrogate models and the total number of actual model computation. Thirdly, an approximate method of computing actual penalty function in the outer loop is proposed for further reducing the computation times of actual simulation model. Computation examples show that the proposed integrated method can greatly decreases the computation times of the actual simulation model and it can obtain accurate optimization results with fast convergence. Hence, the presented method is quite efficient.

\section{Declarations}

\subsection{List of abbreviations}

RBRDO: Reliability-based Robust Design Optimization

PMA: Performance Measure Approach

TR : $\quad$ Trust region

MCS: Monte Carlo simulation

WS : Weighted Sum

PDM: Percentile Difference Method

PZT: Piezoresistor 
An efficient integrated framework for reliability-based robust design optimization of computation-intensive structures

FEM: $\quad$ Finite element model

\subsection{Availability of data and materials}

All data generated or analysed during this study are included in this published article.

\subsection{Competing interests}

The authors declare that they have no competing financial interests.

\subsection{Funding}

The funding is :National Natural Science Foundation of China (Grant No. 51175194) and Promotion Program for Young and Middle-aged Teacher in Science and Technology Research of Huaqiao University (Grant No.ZQN-PY504).

\subsection{Authors' contributions}

Laixiongming: proposes the main thinking efficient integrated framework of reliability-based robust design optimization and guides the paper writing.

Huangju: proposes the approximate estimation of the PMA function, carries out example validation and writes the paper.

Wangcheng: finishes algorithm implementation and participates in paper discussion and modification.

Zhangyong: proposes approximate calculation of the actual penalty function and participates in paper discussion and paper modification. 
An efficient integrated framework for reliability-based robust design optimization of computation-intensive structures

\subsection{Acknowledgements}

The research work is supported by the National Natural Science Foundation of

China (Grant No. 51175194), Promotion Program for Young and Middle-aged Teacher

in Science and Technology Research of Huaqiao University (Grant No.ZQN-PY504).

\section{References}

Ali, S., Ehsan, A., \& Seyed, T. A. N. (2016). A robust loss function approach for a multi-objective redundancy allocation problem. Applied Mathematical Modelling, $\quad 40(1), 1-11$. https://doi.org/https://doi.org/10.1016/j.apm.2015.06.007

Ang, A.-S., \& Tang, W. H. (1984). Probability concepts in engineering planning and design. In Decision, risk and reliability (Vol. 2). Wiley.

Bohlouri, V., \& Jalali-Naini, S. H. (2019). Application of reliability-based robust optimization in spacecraft attitude control with PWPF modulator under uncertainties. Journal of the Brazilian Society of Mechanical Sciences and Engineering, Vol.41 (10), pp.1-15, 1-15. https://doi.org/10.1007/s40430-0191955-9

Castaldo, P., Mancini, G., \& Palazzo, B. (2018). Seismic reliability-based robustness assessment of three-dimensional reinforced concrete systems equipped with single-concave sliding devices. Engineering Structures, 163, 373-387. https://doi.org/10.1016/j.engstruct.2018.02.067

Conn, A. R., Gould, N. I., \& Toint, P. L. (1988). Global convergence of a Class of trust region algorithms for optimization with simple bounds SIAM Journal on Numerical Analysis, 25(2), 433-460. https://doi.org/10.1137/0725029

Dimopoulos, G. G. (2007). Mixed-variable engineering optimization based on evolutionary and social metaphors. Computer Methods in Applied Mechanics and Engineering, 196(4-6), 803-817. https://doi.org/10.1016/j.cma.2006.06.010

Doh, J., Kim, Y., \& Lee, J. (2018). Reliability-based robust design optimization of gap size of annular nuclear fuels using kriging and inverse distance weighting methods. Engineering Optimization, 50(12), 2161-2176. https://doi.org/10.1080/0305215X.2018.1428316

Du, X., Sudjianto, A., \& Chen, W. (2004). An integrated framework for optimization under uncertainty using inverse reliability strategy. ASME, Journal of Mechanical Design, 126(4), 562-570. https://doi.org/10.1115/1.1759358

Fran, S. L., Márcio, A. d. S., Aldemir, A. C. J., \& Valder, S. J. (2020). Reliability-based robust multi-objective optimization applied to engineering system design. Engineering Optimization, $\quad$ 52(1), 1-21. https://doi.org/10.1080/0305215X.2019.1577413

Gang, W., Wang, S., Augenbroe, G., \& Xiao, F. (2016). Robust optimal design of district cooling systems and the impacts of uncertainty and reliability. Energy \& Buildings, 122, 11-22. https://doi.org/10.1016/j.enbuild.2016.04.012 
Gao, X., Tang, H., Huang, P., \& Qin, H. (2020). Robust Design of Low-pressure Casting Process Based on Double Kriging Model. Special casting and nonferrous alloy, 20(6), 595-600. https://doi.org/10.15980/j.tzzz.2020.06.004

Gu, X., \& Lu, J. (2014). Reliability-based robust assessment for multiobjective optimization design of improving occupant restraint system performance. Computers in Industry, 65(8), 1169-1180. https://doi.org/10.1016/j.compind.2014.07.003

Hao, P., Ma, R., Wang, Y., Feng, S., Wang, B., Li, G., Xing, H., \& Yang, F. (2019). An augmented step size adjustment method for the performance measure approach: Toward general structural reliability-based design optimization. Structural Safety, 80, 32-45. https://doi.org/10.1016/j.strusafe.2019.04.001

Javed, A., Raj, J. A., \& Rout, B. (2019). Integrated method for performance analysis of reliability-based topologically optimized components. Proceedings of the institution of mechanical engineers part c-journal of mechanical engineering science, 233(19-20). https://doi.org/10.1177/0954406219865757

Jiang, C., Han, X., \& Liu, G. P. (2008). A sequential nonlinear interval number programming method for uncertain structures. Computer Methods in Applied Mechanics and Engineering, 197(49-50), 4250-4265. https://doi.org/10.1016/j.cma.2008.04.027

Lee, I., Choi, K. K., Du, L., \& Gorsich, D. (2008). Dimension reduction method for reliability-based robust design optimization. Computers \& Structures, 86(1314), 1550-1562. https://doi.org/10.1016/j.compstruc.2007.05.020

Panzeri, M., Savelyev, A., Anisimov, K., d'Ippolito, R., \& Mirzoyan, A. (2018). Uncertainty quantification and robust design optimization applied to aircraft propulsion systems. Transportation Research Procedia, 29, 289-302. https://doi.org/10.1016/j.trpro.2018.02.026

Papadimitriou, D. I., \& Papadimitriou, C. (2016). Aerodynamic shape optimization for minimum robust drag and lift reliability constraint. Aerospace Science and Technology, 55, 24-33. https://doi.org/10.1016/j.ast.2016.05.005

Praveen, C., \& Duvigneau, R. (2009). Low cost PSO using metamodels and inexact preevaluation: Application to aerodynamic shape design. Computer Methods in Applied Mechanics and Engineering, 198(9-12), 1087-1096. https://doi.org/10.1016/j.cma.2008.11.019

Rackwitz, R., \& Flessler, B. (1978). Structural reliability under combined random load sequences. Computers and Structures, 9(5), 489-494. https://doi.org/10.1016/0045-7949(78)90046-9

Rathod, V., Yadav, O. P., Rathore, A., \& Jain, R. (2013). Optimizing reliability-based robust design model using multi-objective genetic algorithm. Computers \& Industrial Engineering, 66(2), 301-310. https://doi.org/10.1016/j.cie.2013.06.018

Rodriguez, J. F., Renaud, J. E., \& Watson, L. T. (1998). Convergence of trust region augmented Lagrangian methods using variable fidelity approximation data. Structural optimization, 15, 141-156. https://doi.org/10.1007/BF01203525

Rosenblatt, M. (1952). Remarks on a multivariate transformation. Annals of Mathematical Statistics, 23(3), 470-472. https://doi.org/10.1214/aoms/1177729394 
Shan, S., \& Wang, G. G. (2008). Reliable design space and complete single-loop reliability-based design optimization. Reliability Engineering \& System Safety, 93(8), 1218-1230. https://doi.org/10.1016/j.ress.2007.07.006

Sun, G., Li, G., Zhou, S., Li, H., Hou, S., \& Li, Q. (2011). Crashworthiness design of vehicle by using multiobjective robust optimization. Structural and Multidisciplinary Optimization, 44, 99-110. https://doi.org/10.1007/s00158-0100601-Z

Tsao, Y.-C., \& Thanh, V.-V. (2020). A multi-objective fuzzy robust optimization approach for designing sustainable and reliable power systems under uncertainty. Applied Soft Computing Journal, 92, 1-14. https://doi.org/10.1016/j.asoc.2020.106317

Wang, G. G. (2003). Adaptive response surface method using inherited latin hypercube design points. J. Mech. Des., Trans. ASME, 125, 210-220. https://doi.org/10.1115/1.1561044

Wu, P., Gao, L., Zou, D., \& Li, S. (2011). An improved particle swarm optimization algorithm for reliability problems. 2011, 50(1), 71-81. https://doi.org/10.1016/j.isatra.2010.08.005

Yang, M., Zhang, D., \& Han, X. (2020). New efficient and robust method for structural reliability analysis and its application in reliability-based design optimization. Computer Methods in Applied Mechanics and Engineering, 366, 1-24. https://doi.org/10.1016/j.cma.2020.113018

Yu, H., Gillot, F., \& Ichchou, M. (2013). Reliability based robust design optimization for tuned mass damper in passive vibration control of deterministic/uncertain structures. Journal of Sound and Vibration, 332(9), 2222-2238. https://doi.org/10.1016/j.jsv.2012.12.014

Zhu, P., Zhang, Y., \& Chen, G. L. (2009). Metamodel-based lightweight design of automotive front-body structure using robust optimization. Journal Automobile Engineering, 223, 1133-1147. https://doi.org/10.1243/09544070JAUTO1045 
An efficient integrated framework for reliability-based robust design optimization of computation-intensive structures

\section{Table \& Figure Captions}

Table 1

Input parameters for the beam design problem.

Table 2

Optimization results under with different starting design points.

Table 3

Optimization results under with different TR radii.

Table 4

Optimization results under different uncertainty levels of the design variables.

Table 5

Optimization results under different uncertainty levels of the random variables.

Table 6

Input parameters for the 72-bar truss design problem.

Table 7

Optimization results of the 72-bar truss (m).

Table 8

Input parameters for the pressure sensor design problem.

Table 9

Optimization results of the pressure sensor $\left(\mathrm{m}^{-3}\right)$.

Fig. 1. The procedure of double-loop optimization for the general RBRDO.

Fig. 2 The distribution of the performance function $f$.

Fig. 3. The procedure of the double-loop optimization for the improved RBRDO formulation. 
An efficient integrated framework for reliability-based robust design optimization of computation-intensive structures

Fig. 4 The efficient sequential approximate RBRDO.

Fig. 5 Computation of the actual penalty function at the specified design vector $\mathbf{V}$.

Fig. 6 A beam design problem.

Fig. 7 Iterative optimization process with different starting design points.

Fig. 8 Iterative trajectory with different TR radii.

Fig. 9 A 72-bar truss.

Fig. 10 Iteration history of the 72-bar truss.

Fig. 11. Schema of piezoresistive pressure sensor.

Fig.12 The main sizes of pressure sensor.

Fig. 13 Iteration history of the pressure sensor design. 
An efficient integrated framework for reliability-based robust design optimization of computation-intensive structures

\section{Table}

Table 1

Input parameters for the beam design problem.

\begin{tabular}{|c|c|c|c|c|}
\hline Name & Mean Value & $\begin{array}{l}\text { Range of the } \\
\text { variation }(\xi)\end{array}$ & $\begin{array}{l}\text { Standard } \\
\text { Deviation }\end{array}$ & Distribution Type \\
\hline$d_{l}(\mathrm{~cm})$ & $\mu_{d 1}$ & $\pm d_{1}^{L} / 1000$ & & Normal \\
\hline \multirow[t]{2}{*}{$d_{2}(\mathrm{~cm})$} & $\mu_{d 2}$ & $\pm d_{2}^{L} / 1000$ & & Normal \\
\hline & & & $|\xi| / 3$ & \\
\hline$X_{I}(\mathrm{~cm})$ & 1 & \pm 0.1 & & Normal \\
\hline$X_{2}(\mathrm{~cm})$ & 2 & \pm 0.2 & & Normal \\
\hline $\begin{array}{l}\text { Young's Modulus } \\
E\left(\mathrm{kN} / \mathrm{cm}^{2}\right)\end{array}$ & $2 \times 10^{4}$ & ---- & ---- & ---- \\
\hline $\begin{array}{l}\text { Bending force } P \\
(\mathrm{kN})\end{array}$ & 600 & ---- & ---- & ---- \\
\hline $\begin{array}{l}\text { Bending force } Q \\
(\mathrm{kN})\end{array}$ & 50 & ---- & ---- & ---- \\
\hline Length $L(\mathrm{~cm})$ & 200 & --- & --- & ---- \\
\hline
\end{tabular}

Table 2

Optimization results under with different starting design points.

$\begin{array}{ccccc} & & & & \\ \\ \text { Iteration number }\end{array}$


An efficient integrated framework for reliability-based robust design optimization of computation-intensive structures

$\begin{array}{ccccc}7 & (58.13,37.50) & (80.00,50.00) & (80.00,50.00) & (80.00,50.00) \\ 8 \sim 20 & (80.00,50.00) & (80.00,50.00) & (80.00,50.00) & (80.00,50.00)\end{array}$

Table 3

Optimization results under with different TR radii.

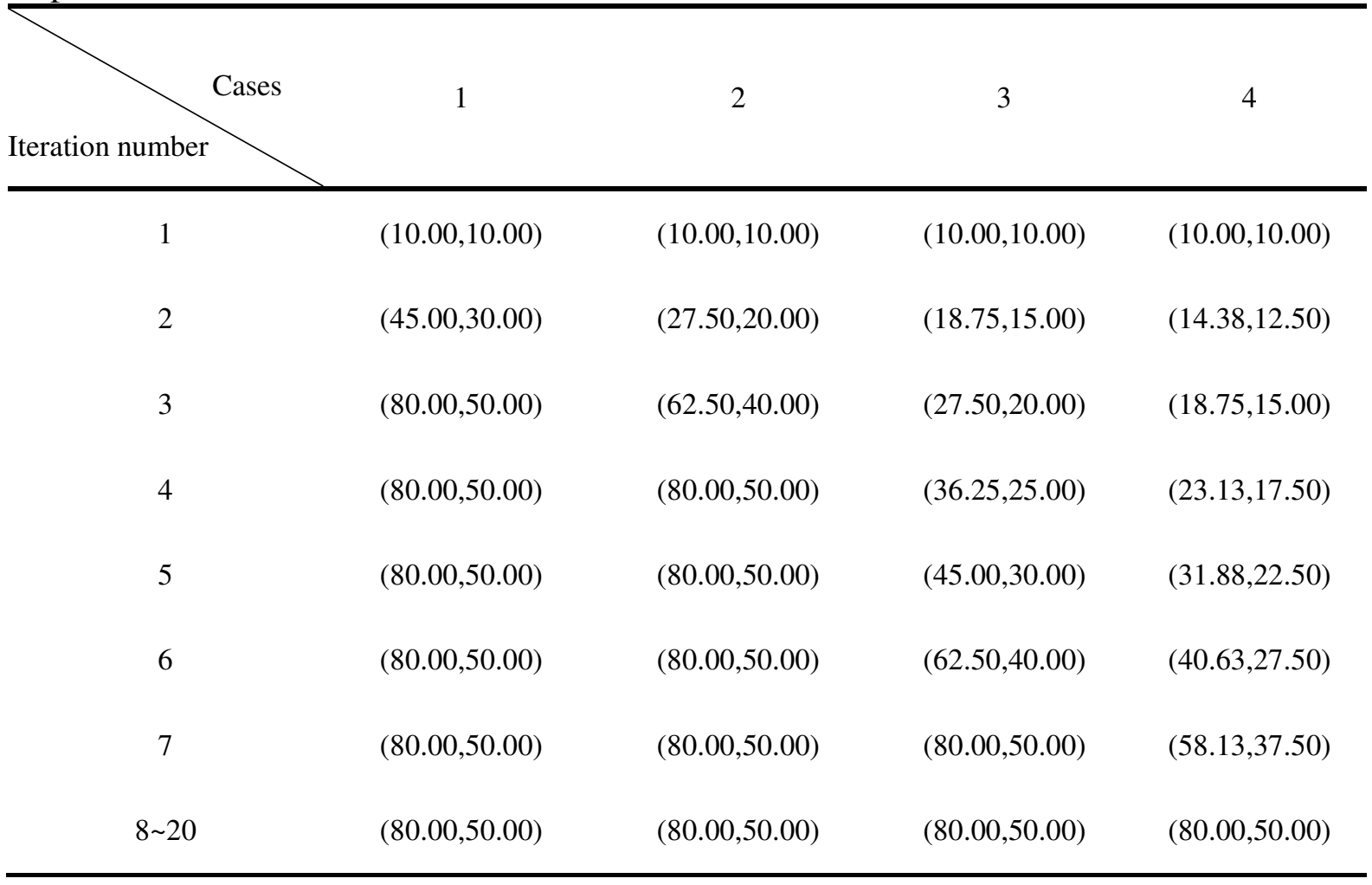

Table 4

Optimization results under different uncertainty levels of the design variables.

\begin{tabular}{|c|c|c|c|c|c|c|c|c|}
\hline $\begin{array}{l}\text { Variation } \\
\text { range } \xi\end{array}$ & $\pm \frac{\mathrm{d}^{L}}{10^{0}}$ & $\pm \frac{\mathrm{d}^{L}}{10^{-1}}$ & $\pm \frac{\mathrm{d}^{L}}{10^{-2}}$ & $\pm \frac{\mathrm{d}^{L}}{10^{-3}}$ & $\pm \frac{\mathbf{d}^{L}}{10^{4}} \quad \pm \frac{\mathbf{d}^{L}}{10^{-5}}$ & $\pm \frac{\mathrm{d}^{L}}{10^{-6}}$ & $\pm \frac{d^{L}}{10^{-7}}$ & $\pm \frac{\mathrm{d}^{L}}{10^{-8}}$ \\
\hline $\begin{array}{c}\text { Standard } \\
\text { Deviation }\end{array}$ & & & & & $\|\xi\| / 3$ & & & \\
\hline $\begin{array}{l}\text { Optimum } \\
\text { results }\end{array}$ & $\begin{array}{l}(80.00 \\
44.31)\end{array}$ & & & & $(80.00,50.00)$ & & & \\
\hline
\end{tabular}

Table 5

Optimization results under different uncertainty levels of the random variables.

\begin{tabular}{|c|c|c|c|c|c|}
\hline Ratio & Optimum results & $H_{p}$ & $\begin{array}{l}\text { Precise optimum } \\
\text { results }\end{array}$ & $\begin{array}{c}\text { Precise } \\
H_{p}\end{array}$ & $\begin{array}{l}\text { Deviations from the } \\
\text { precise optimum }\end{array}$ \\
\hline
\end{tabular}


An efficient integrated framework for reliability-based robust design optimization of computation-intensive structures

\begin{tabular}{lccccc}
\hline $\pm 10 \%$ & $(80.00,50.00)$ & 0.72 & $(80.00,50.00)$ & 0.60 & $(0 \%, 0 \%)$ \\
$\pm 20 \%$ & $(80.00,50.00)$ & 1.00 & $(80.00,50.00)$ & 0.71 & $(0 \%, 0 \%)$ \\
$\pm 30 \%$ & $(80.00,49.36)$ & 1.37 & $(80.00,49.35)$ & 0.81 & $(0 \%, 0 \%)$ \\
$\pm 40 \%$ & $(78.62,47.76)$ & 1.94 & $(80.00,47.44)$ & 0.93 & $(1.72 \%, 0.67 \%)$ \\
$\pm 50 \%$ & $(74.53,46.88)$ & 2.57 & $(80.00,45.66)$ & 2.56 & $(0.06 \%, 0.03 \%)$ \\
$\pm 60 \%$ & $(80.00,43.99)$ & 3.51 & $(77.75,44.40)$ & 1.55 & $(2.9 \%, 0.91 \%)$ \\
$\pm 70 \%$ & $(80.00,42.42)$ & 38454 & $(58.83 \%, 33.97 \%)$ & 11.71 & $(35.98 \%, 24.87 \%)$ \\
$\pm 80 \%$ & $(80.00,40.95)$ & 124900 & $(50.15 \%, 45.50 \%)$ & 4.38 & $(59.52 \%, 10.02 \%)$ \\
$\pm 90 \%$ & $(80.00,39.55)$ & 222727 & $(55.03 \%, 33.61 \%)$ & 4.88 & $(45.37 \%, 17.68 \%)$ \\
\hline
\end{tabular}

Table 6

Input parameters for the 72-bar truss design problem.

\begin{tabular}{ccccc}
\hline Name & Mean Value & $\begin{array}{c}\text { Range of the } \\
\text { variation }(\xi)\end{array}$ & $\begin{array}{c}\text { Standard } \\
\text { Deviation }\end{array}$ & Distribution Type \\
\hline$H(\mathrm{~m})$ & $\mu_{H}$ & $\pm H^{L} / 1000$ & & Normal \\
$B(\mathrm{~m})$ & $\mu_{B}$ & $\pm B^{L} / 1000$ & Normal \\
$t(\mathrm{~m})$ & $\mu_{h}$ & $\pm t^{L} / 1000$ & Normal \\
$\begin{array}{c}\text { Young's Modulus } \\
E(\mathrm{MPa})\end{array}$ & $2.1 \times 10^{5}$ & $\pm 6.3 \times 10^{4}$ & $\mid \xi / 3$ & Normal \\
$\begin{array}{c}\text { Density } \rho \\
\left(\mathrm{kg} / \mathrm{m}^{3}\right)\end{array}$ & 7860 & \pm 2358 & & Normal \\
$\begin{array}{c}\text { The concentrated } \\
\text { force } P(\mathrm{kN})\end{array}$ & 100 & \pm 30 & Normal \\
\hline
\end{tabular}

Table 7

Optimization results of the 72-bar truss (m).

\begin{tabular}{cccccccc}
\hline $\begin{array}{c}\text { Iteration } \\
\text { number } \\
k\end{array}$ & $\begin{array}{c}\text { Design vector } \\
\boldsymbol{\mu}_{\mathbf{d}}^{k}\end{array}$ & $\begin{array}{c}\text { Penalty } \\
\text { function } \\
H_{p}\left(\boldsymbol{\mu}_{\mathrm{d}}^{k}\right)\end{array}$ & $\mu_{f}$ & $f_{0.00135}$ & $f_{0.99865}$ & $g_{1}\left(\mathbf{u}_{1}^{*} ; \mathbf{V}\right)$ & $g_{2}\left(\mathbf{u}_{2}^{*} ; \mathbf{V}\right)$ \\
\hline
\end{tabular}


An efficient integrated framework for reliability-based robust design optimization of computation-intensive structures

\begin{tabular}{ccccccccc}
\hline 1 & $(15,15,5)$ & $2.48 \times 10^{7}$ & 139.23 & 97.46 & 181.00 & -247.18 & $9.90 \times 10^{-4}$ \\
2 & $(16.5,16.5,5.5)$ & $1.79 \times 10^{7}$ & 168.47 & 117.93 & 219.01 & -178.25 & 0.0013 \\
3 & $(19.5,19.5,6.5)$ & $8.60 \times 10^{6}$ & 235.30 & 164.71 & 305.89 & -85.02 & 0.0018 \\
4 & $(25.35,20.52,8.47)$ & $1.36 \times 10^{6}$ & 338.67 & 237.07 & 440.27 & -13.28 & 0.0022 \\
5 & $(31.35,19.30,6.90)$ & $4.86 \times 10^{5}$ & 357.32 & 250.12 & 464.51 & -4.76 & 0.0022 \\
$6 \sim 7$ & $(36.42,18.29,6.84)$ & $2.25 \times 10^{5}$ & 363.21 & 254.25 & 472.17 & -2.25 & 0.0022 \\
8 & $(36.79,17.91,6.71)$ & $1.68 \times 10^{5}$ & 364.60 & 255.22 & 473.98 & -1.67 & 0.0022 \\
9 & $(36.41,17.54,6.59)$ & 4695.4 & 368.56 & 257.99 & 479.12 & -0.0446 & 0.0022 \\
$10 \sim 20$ & $(36.36,17.49,6.57)$ & 239.64 & 368.68 & 258.07 & 479.28 & 0.0050 & 0.0022 \\
\hline & & & & & & & & \\
\hline
\end{tabular}

Table 8

Input parameters for the pressure sensor design problem.

\begin{tabular}{ccccc}
\hline Name & Mean Value & $\begin{array}{c}\text { Range of the } \\
\text { variation }(\xi)\end{array}$ & $\begin{array}{c}\text { Standard } \\
\text { Deviation }\end{array}$ & Distribution Type \\
\hline$a(\mathrm{~m})$ & $\mu_{a}$ & $\pm a^{L} / 1000$ & & Normal \\
$b(\mathrm{~m})$ & $\mu_{b}$ & $\pm b^{L} / 1000$ & Normal \\
$c(\mathrm{~m})$ & $\mu_{c}$ & $\pm c^{L} / 1000$ & Normal \\
$d(\mathrm{~m})$ & $\mu_{d}$ & $\pm d^{L} / 1000$ & Normal \\
$\begin{array}{c}\text { Density } \\
\rho\left(\mathrm{kg} / \mathrm{m}^{3}\right)\end{array}$ & $2.34 \times 10^{3}$ & \pm 702 & Normal \\
$\begin{array}{c}\text { Young's Modulus } \\
E(\mathrm{MPa})\end{array}$ & $1.689 \times 10^{11}$ & $\pm 5.067 \times 10^{10}$ & & Normal \\
$\begin{array}{c}\text { Poisson's ratio Nu } \\
\text { The pressure } P \\
(\mathrm{Mpa})\end{array}$ & 0.0624 & \pm 0.01872 & Normal \\
\hline
\end{tabular}

Table 9 
An efficient integrated framework for reliability-based robust design optimization of computation-intensive structures

Optimization results of the pressure sensor $\left(\mathrm{m}^{-3}\right)$.

\begin{tabular}{|c|c|c|c|c|c|c|c|}
\hline $\begin{array}{c}\text { Iteration } \\
\text { number } \\
k\end{array}$ & $\begin{array}{c}\text { Design vector } \\
\boldsymbol{\mu}_{\mathrm{d}}^{k}\end{array}$ & $\begin{array}{c}\text { Penalty } \\
\text { function } \\
H_{p}\left(\boldsymbol{\mu}_{\mathrm{d}}^{k}\right)\end{array}$ & $\mu_{f}$ & $f_{0.00135}$ & $f_{0.99865}$ & $g_{1}\left(\mathbf{u}_{1}^{*} ; \mathbf{V}\right)$ & $g_{2}\left(\mathbf{u}_{2}^{*} ; \mathbf{V}\right)$ \\
\hline 1 & $(0.5,0.01,0.1,0.3)$ & $1.87 \times 10^{10}$ & 88.14 & 61.70 & 114.61 & 0.044 & $-1.87 \times 10^{4}$ \\
\hline 2 & $\begin{array}{c}(0.54955,0.0105,0 \\
.1,0.3)\end{array}$ & $1.47 \times 10^{10}$ & 96.64 & 67.64 & 125.66 & 0.043 & $-1.47 \times 10^{4}$ \\
\hline 3 & $\begin{array}{c}(0.59885,0.0105,0 \\
.1,0.3)\end{array}$ & $1.06 \times 10^{10}$ & 114.90 & 80.42 & 149.40 & 0.040 & $-1.06 \times 10^{4}$ \\
\hline 4 & $\begin{array}{c}(0.69755,0.0125,0 \\
.1,0.36)\end{array}$ & $7.77 \times 10^{9}$ & 109.96 & 76.97 & 142.98 & 0.039 & -7772.62 \\
\hline 5 & $\begin{array}{c}(0.79555,0.0145,0 \\
.1,0.3)\end{array}$ & $5.61 \times 10^{9}$ & 106.66 & 74.24 & 137.91 & 0.038 & -5612.81 \\
\hline 6 & $\begin{array}{c}(0.8935,0.0125,0 . \\
14,0.3)\end{array}$ & $1.13 \times 10^{8}$ & 180.37 & 126.25 & 234.53 & 0.021 & -112.79 \\
\hline $7 \sim 13$ & $\begin{array}{c}(1.090,0.0165,0.1 \\
4,0.3)\end{array}$ & 100.14 & 154.03 & 107.81 & 200.28 & 0.022 & 1282.44 \\
\hline $14 \sim 16$ & $\begin{array}{c}(1.084,0.0166,0.1 \\
3875,0.3)\end{array}$ & 97.51 & 149.98 & 104.98 & 195.02 & 0.023 & 1079.736 \\
\hline \multicolumn{8}{|c|}{ Total number of the FEM evaluations is 1280} \\
\hline
\end{tabular}


An efficient integrated framework for reliability-based robust design optimization of computation-intensive structures

\section{Figure}

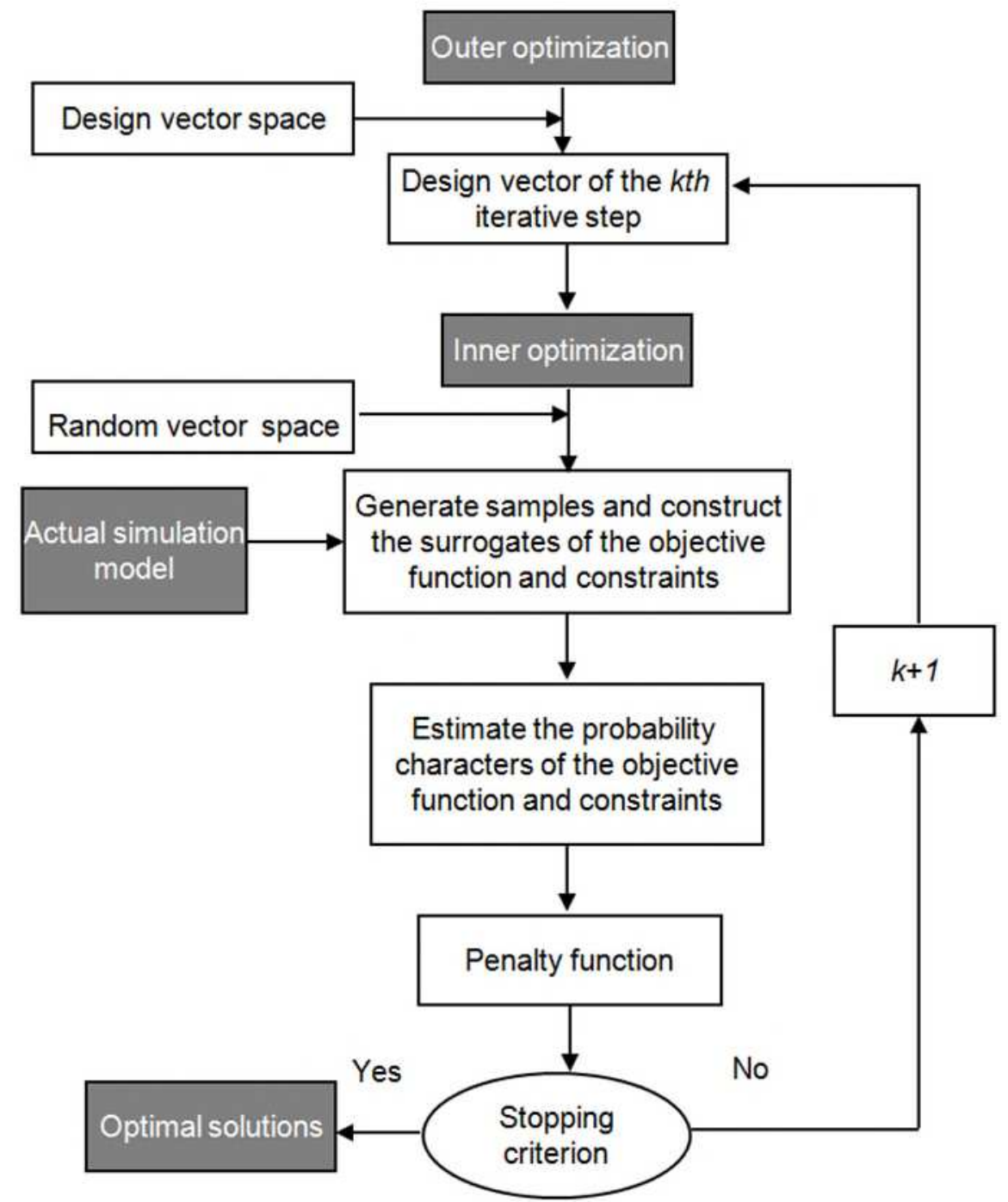

Fig. 1. The procedure of double-loop optimization for the general RBRDO.

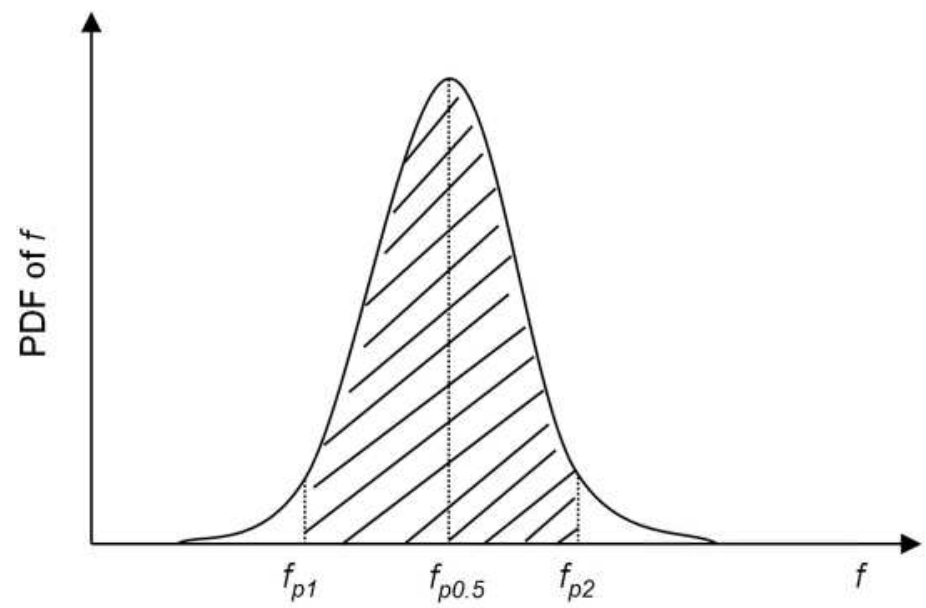


An efficient integrated framework for reliability-based robust design optimization of computation-intensive structures

Fig. 2 The distribution of the performance function $f$.

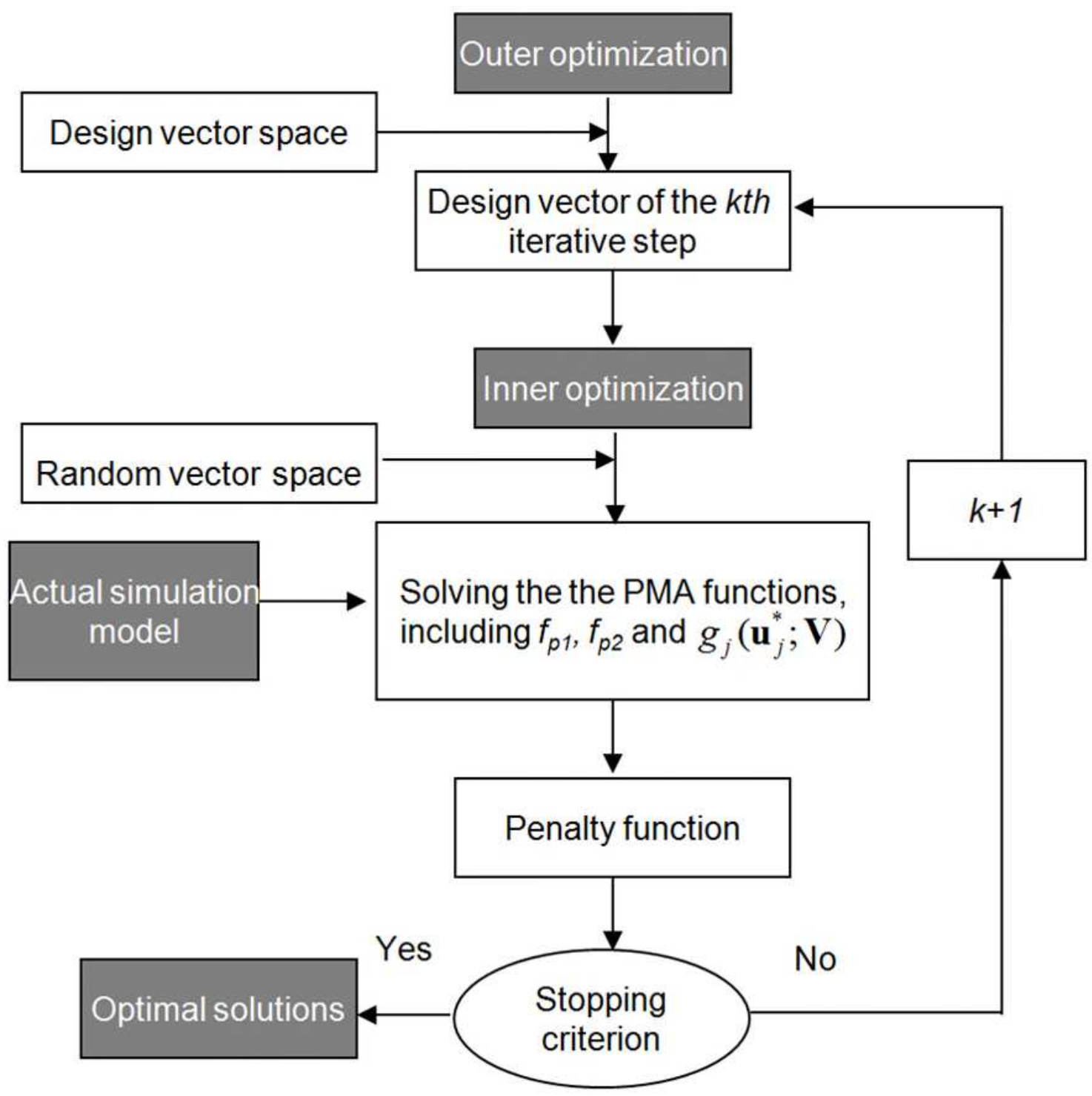

Fig. 3. The procedure of the double-loop optimization for the improved RBRDO formulation. 
An efficient integrated framework for reliability-based robust design optimization of computation-intensive structures

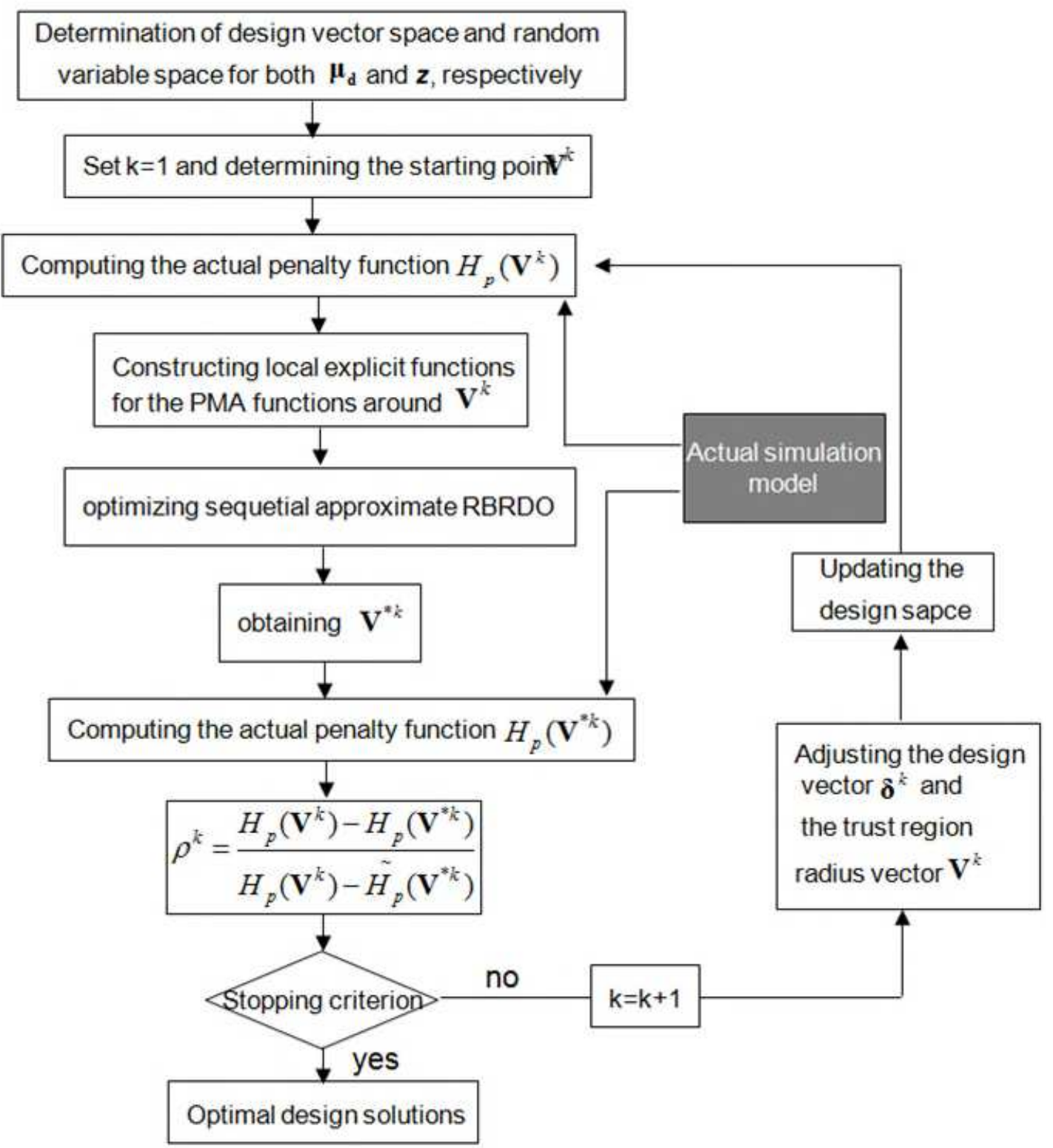

Fig. 4 The efficient sequential approximate RBRDO. 
An efficient integrated framework for reliability-based robust design optimization of computation-intensive structures

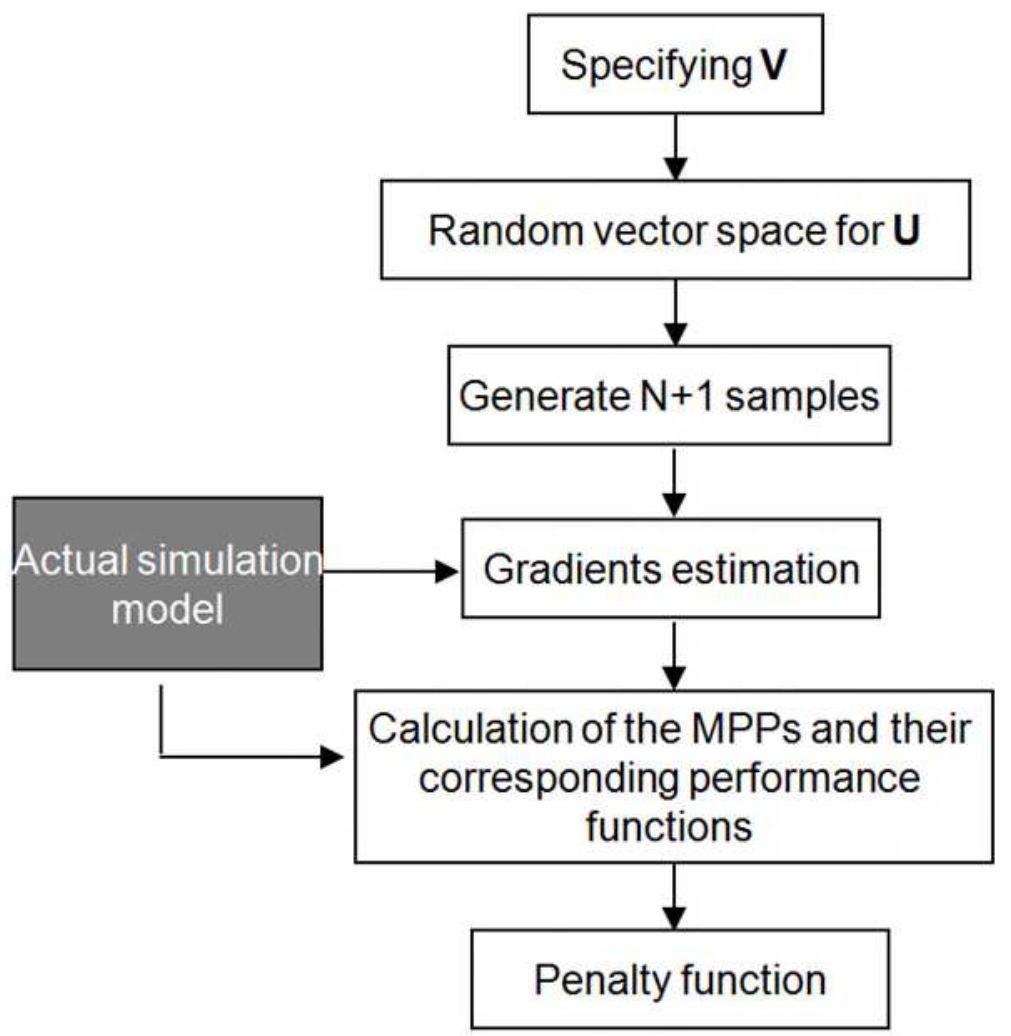

Fig. 5 Computation of the actual penalty function at the specified design vector $\mathbf{V}$.
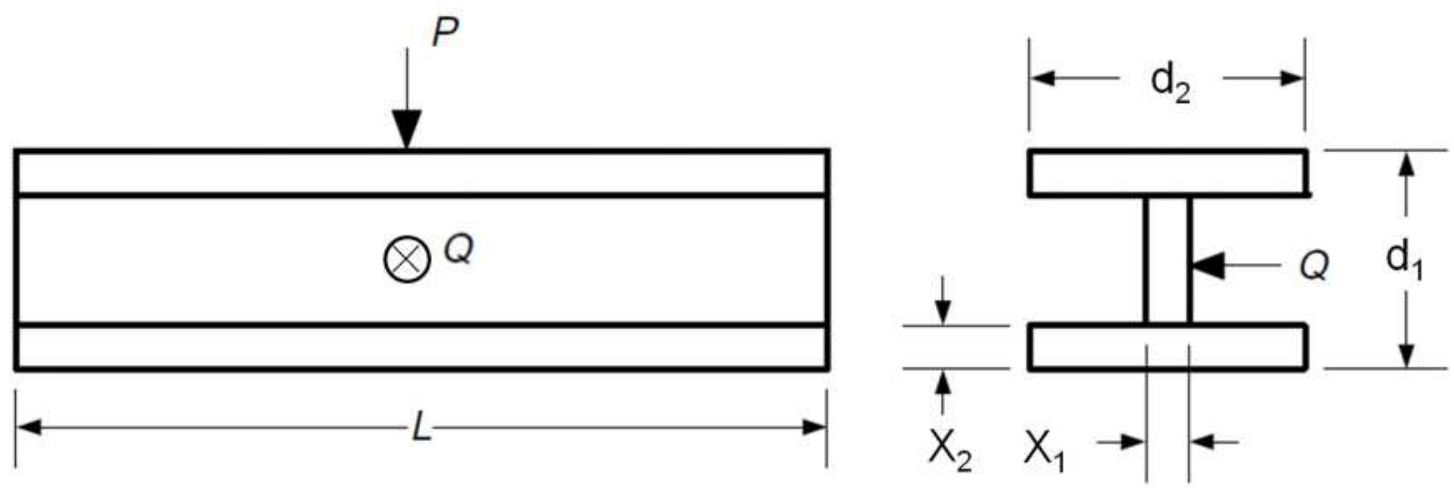

Fig. 6 A beam design problem. 
An efficient integrated framework for reliability-based robust design optimization of computation-intensive structures

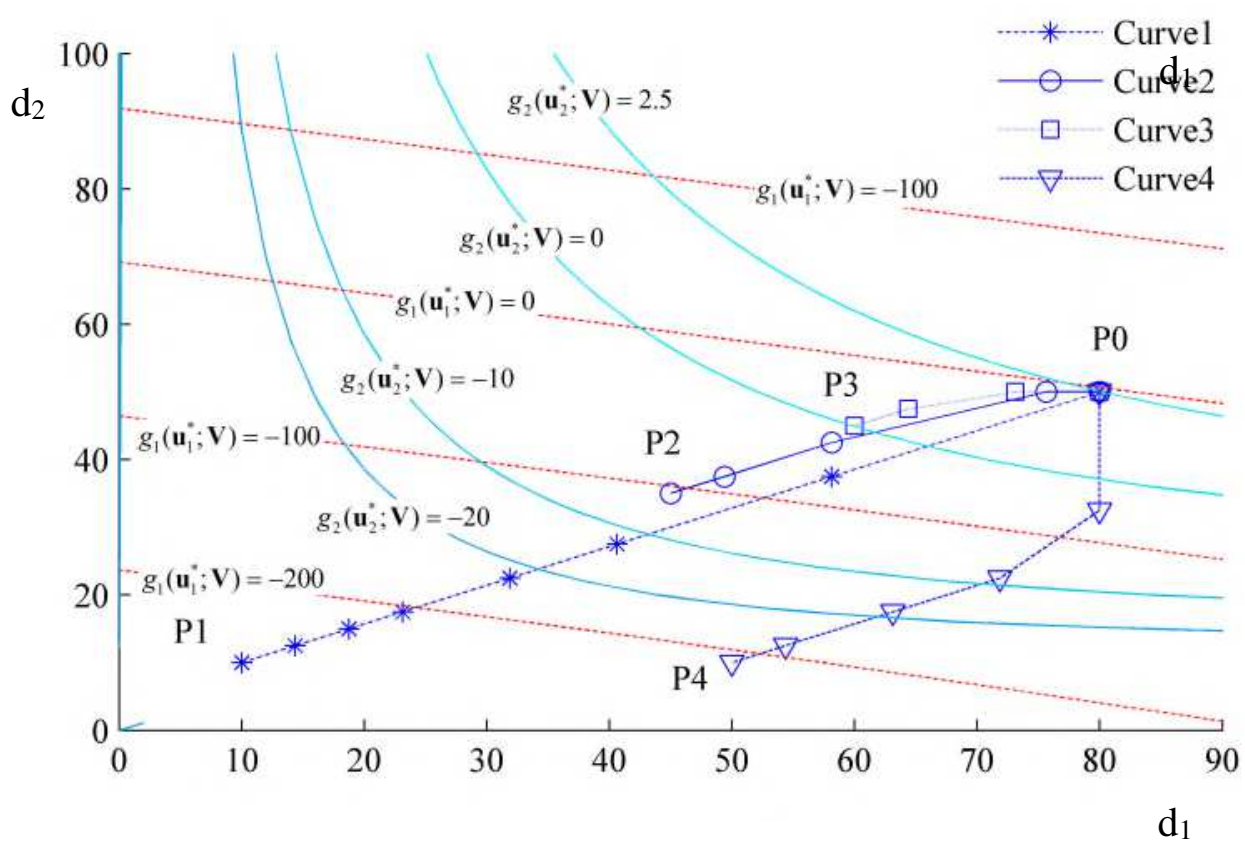

Fig. 7 Iterative optimization process with different starting design points.

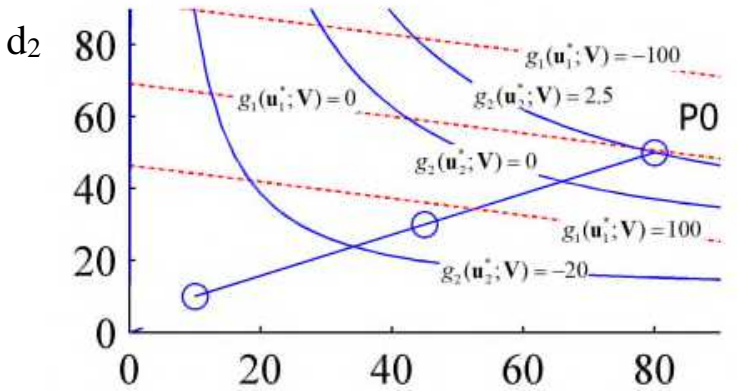

(a)

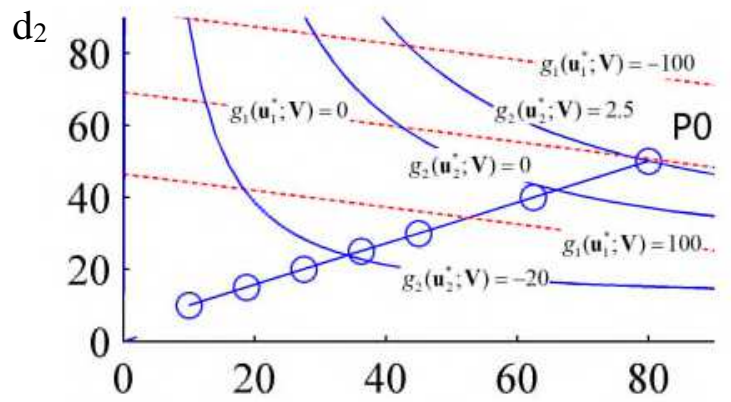

(c)

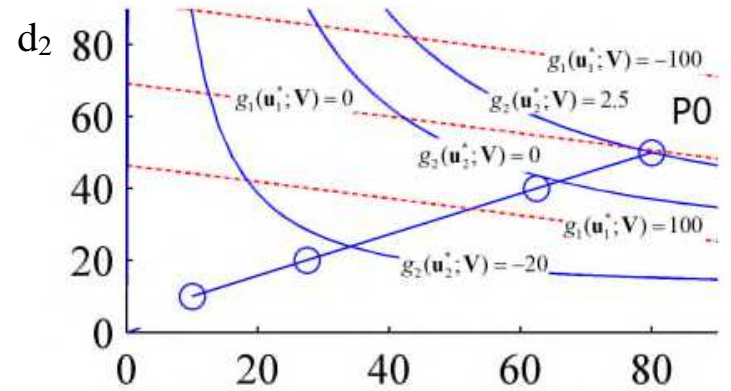

(b)

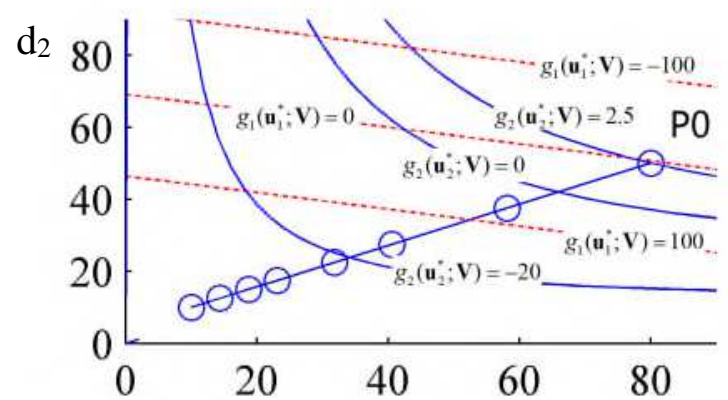

(d)

$\mathrm{d}_{1}$

Fig. 8 Iterative trajectory with different TR radii. 

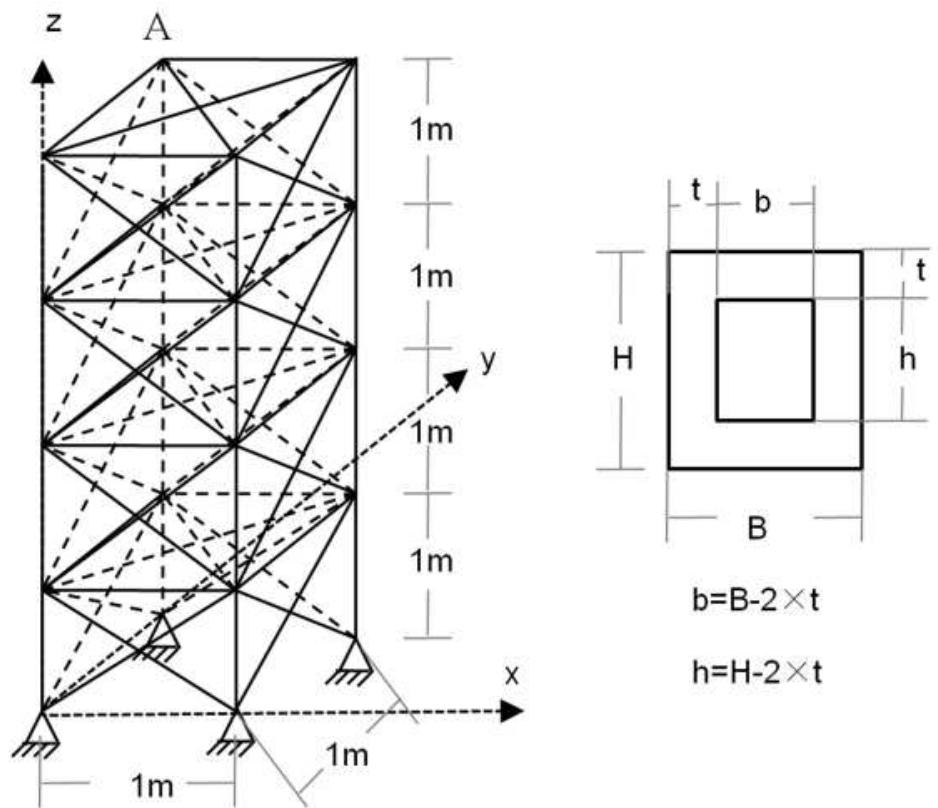

$\mathrm{b}=\mathrm{B}-2 \times \mathrm{t}$

$\mathrm{h}=\mathrm{H}-2 \times \mathrm{t}$

Fig. 9 A 72-bar truss.

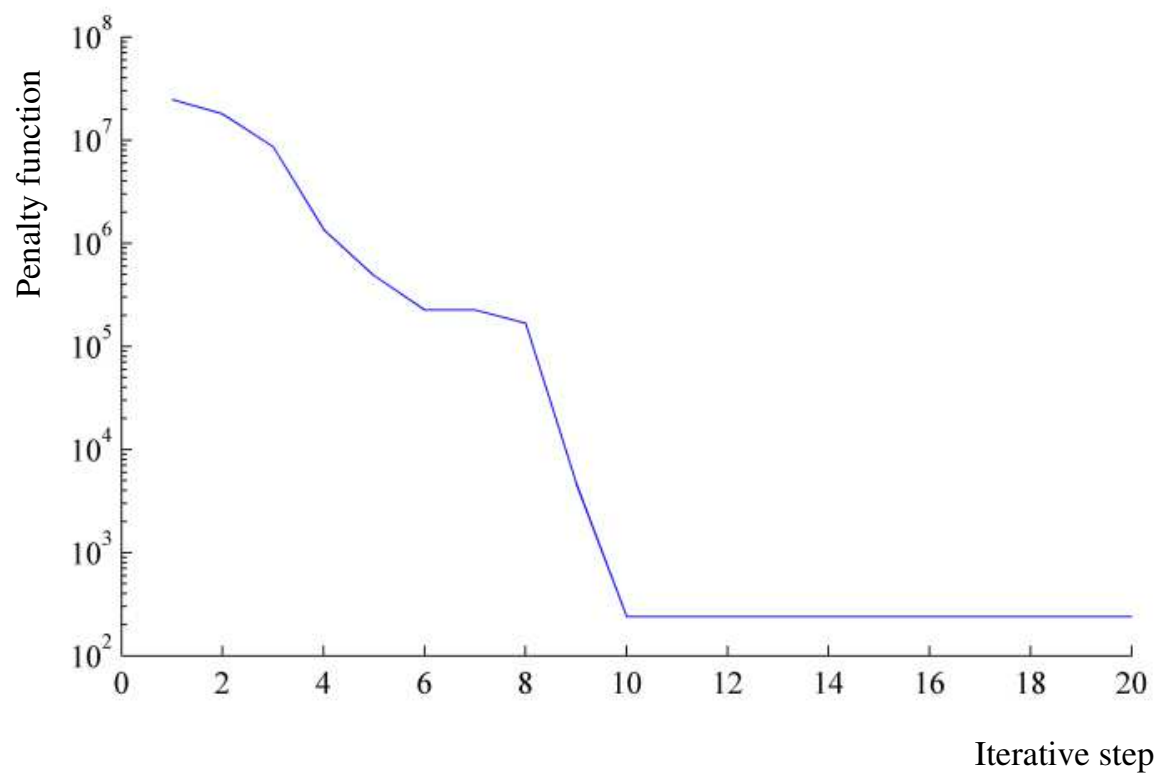

Fig. 10 Iteration history of the 72-bar truss.

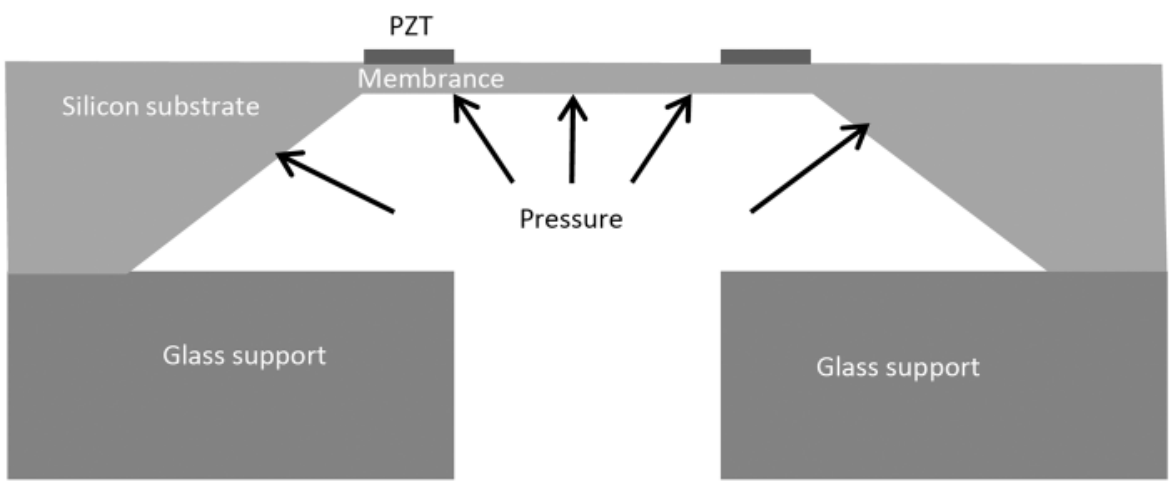


An efficient integrated framework for reliability-based robust design optimization of computation-intensive structures

Fig. 11. Schema of piezoresistive pressure sensor.

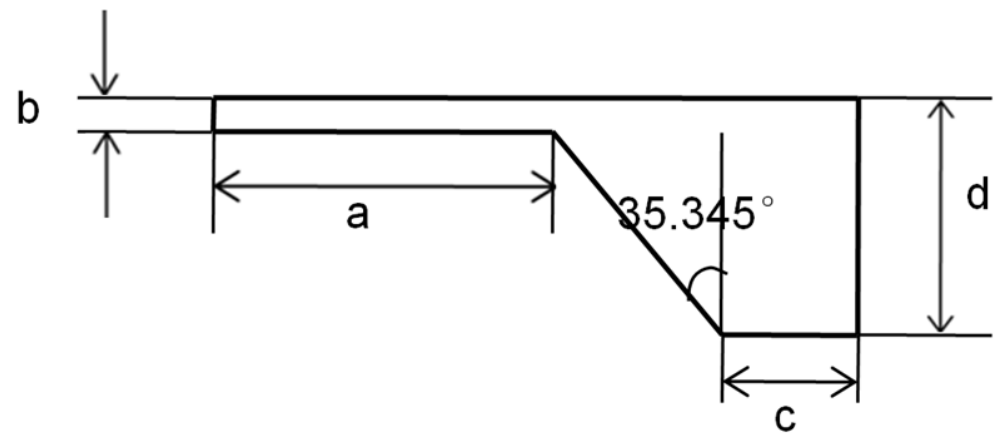

Fig.12 The main sizes of pressure sensor.

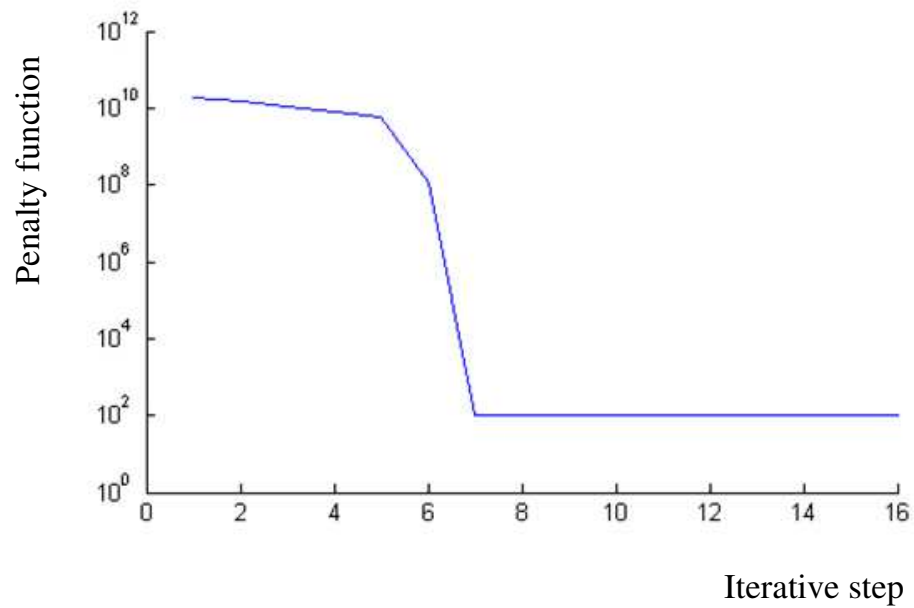

Fig. 13 Iteration history of the pressure sensor design. 
Figures

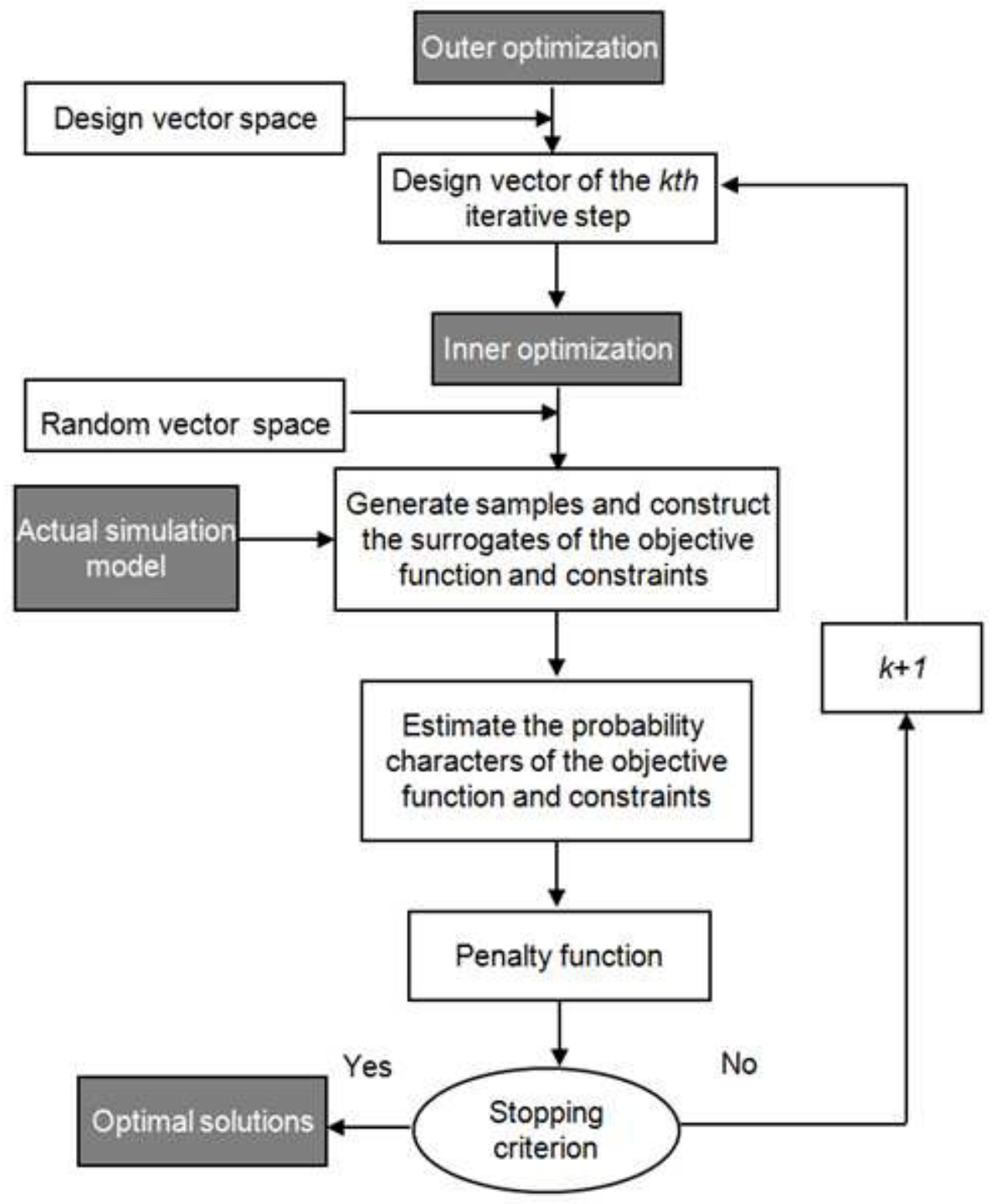

Figure 1

The procedure of double-loop optimization for the general RBRDO. 


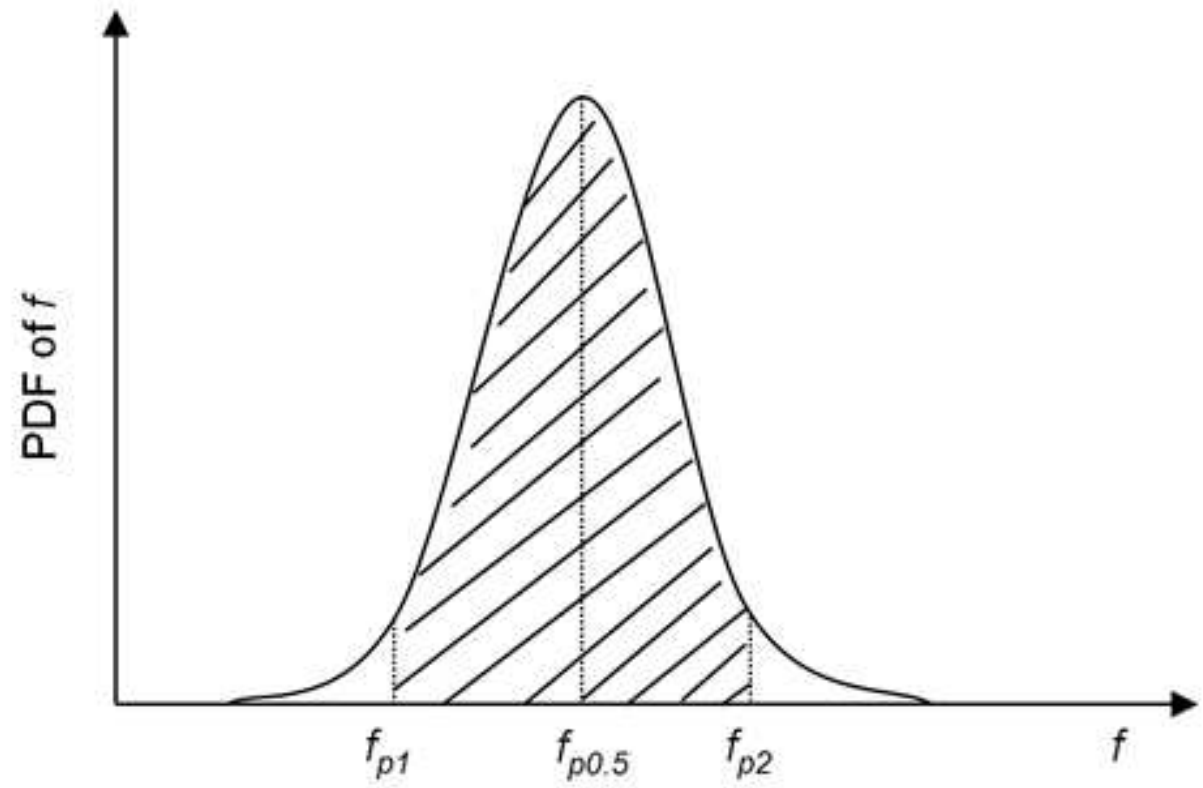

Figure 2

The distribution of the performance function $\mathrm{f}$. 


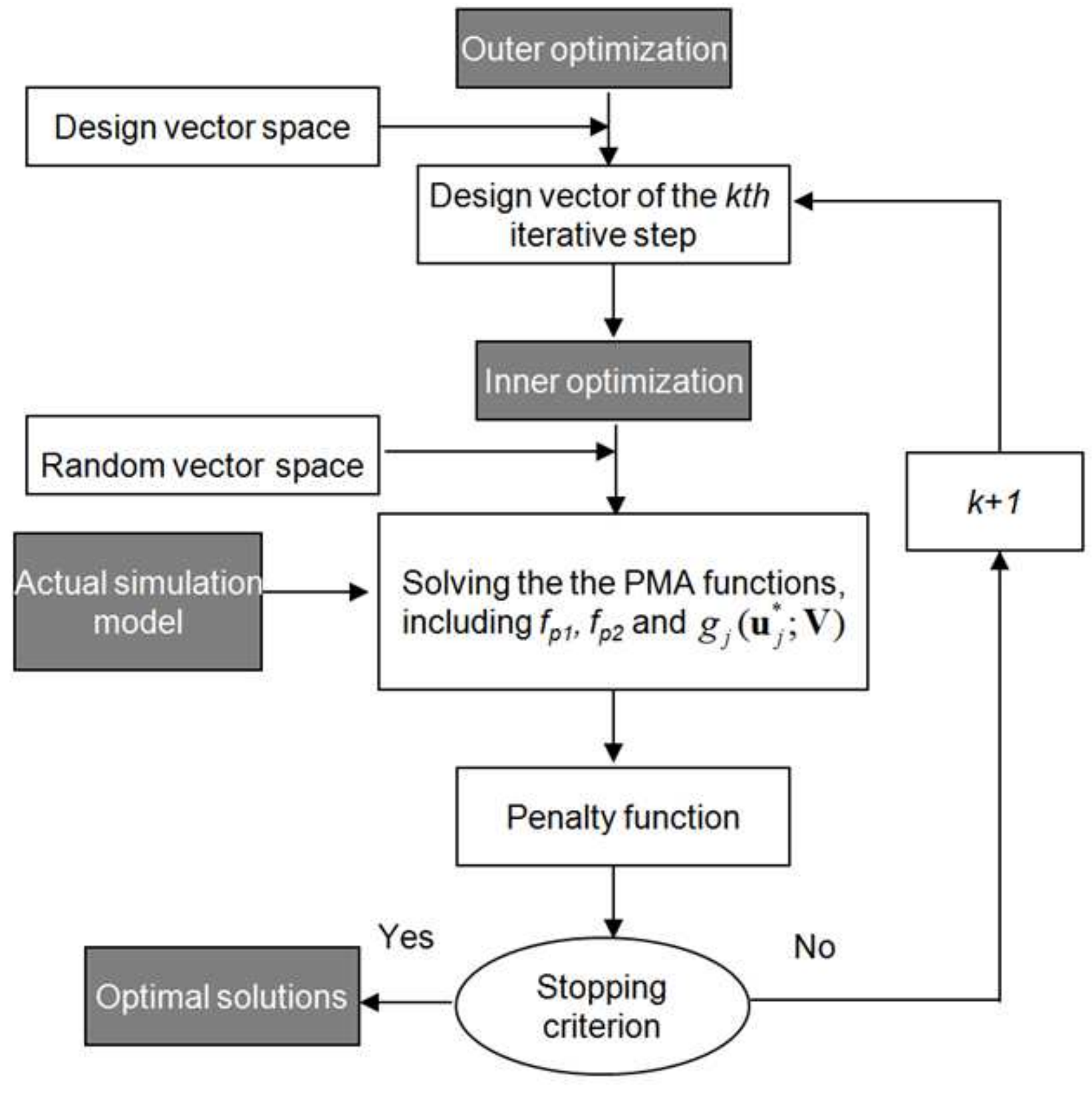

Figure 3

The procedure of the double-loop optimization for the improved RBRDO formulation. 


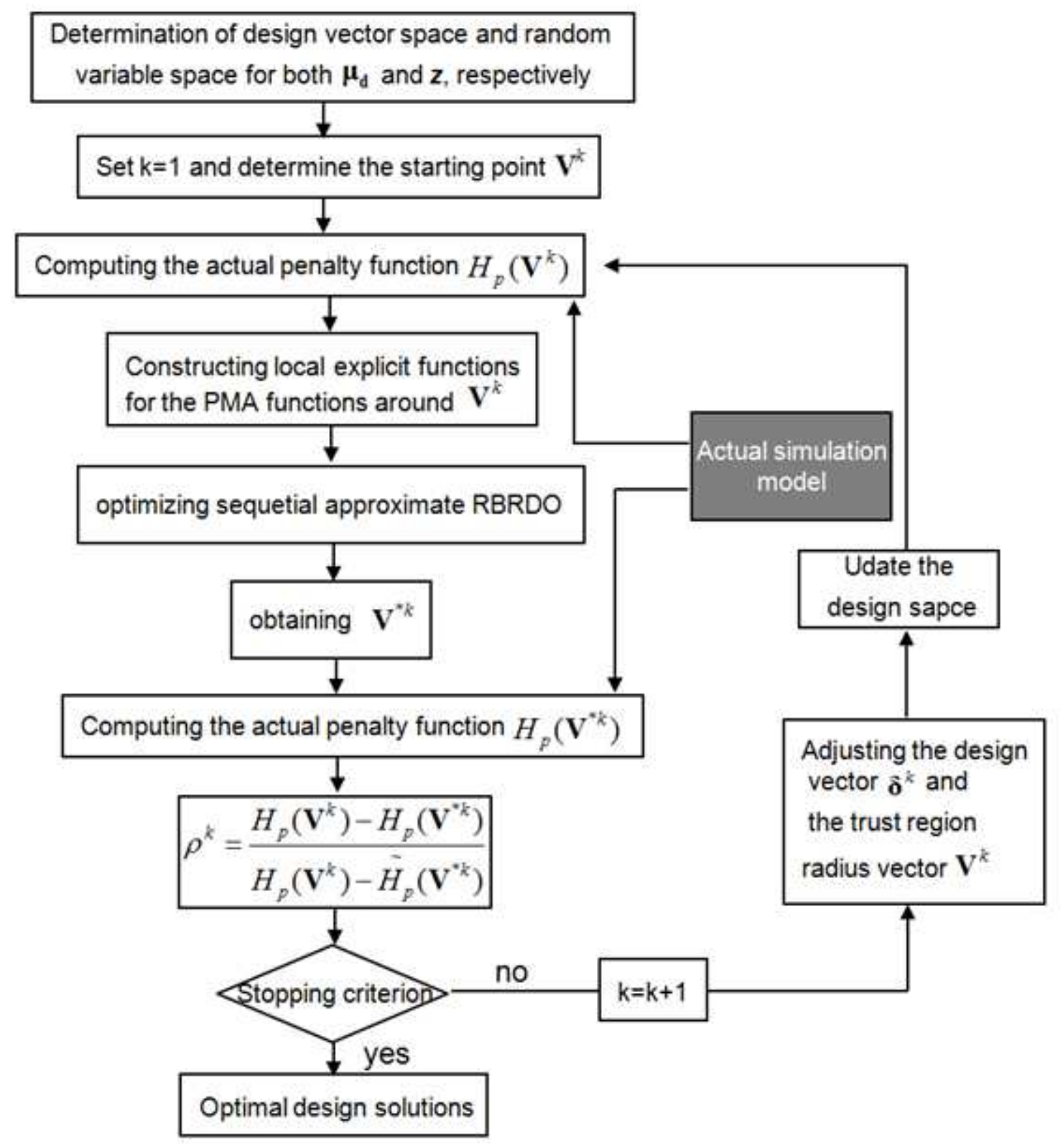

Figure 4

The efficient sequential approximate RBRDO. 


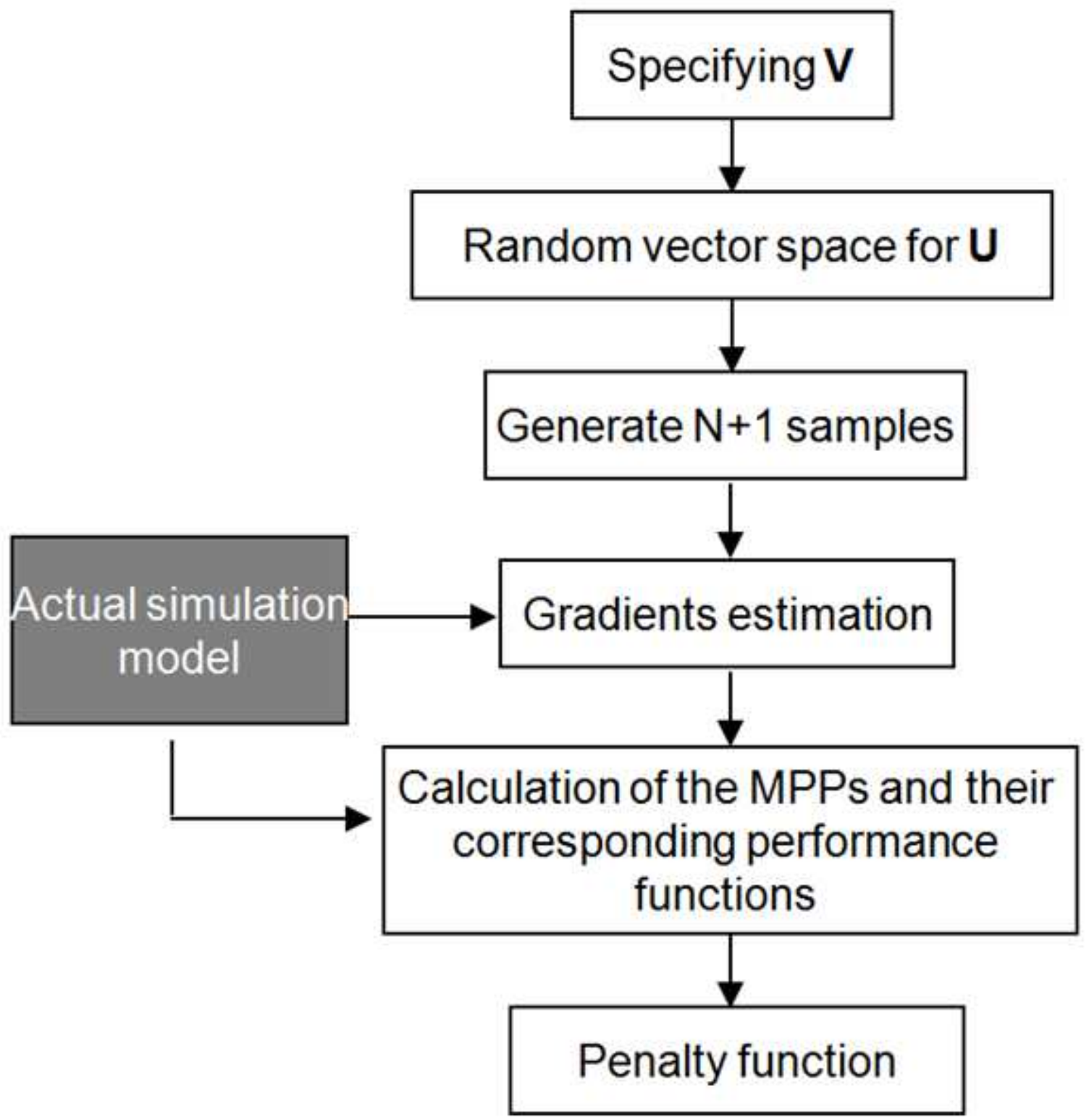

Figure 5

Computation of the actual penalty function at the specified design vector V. 


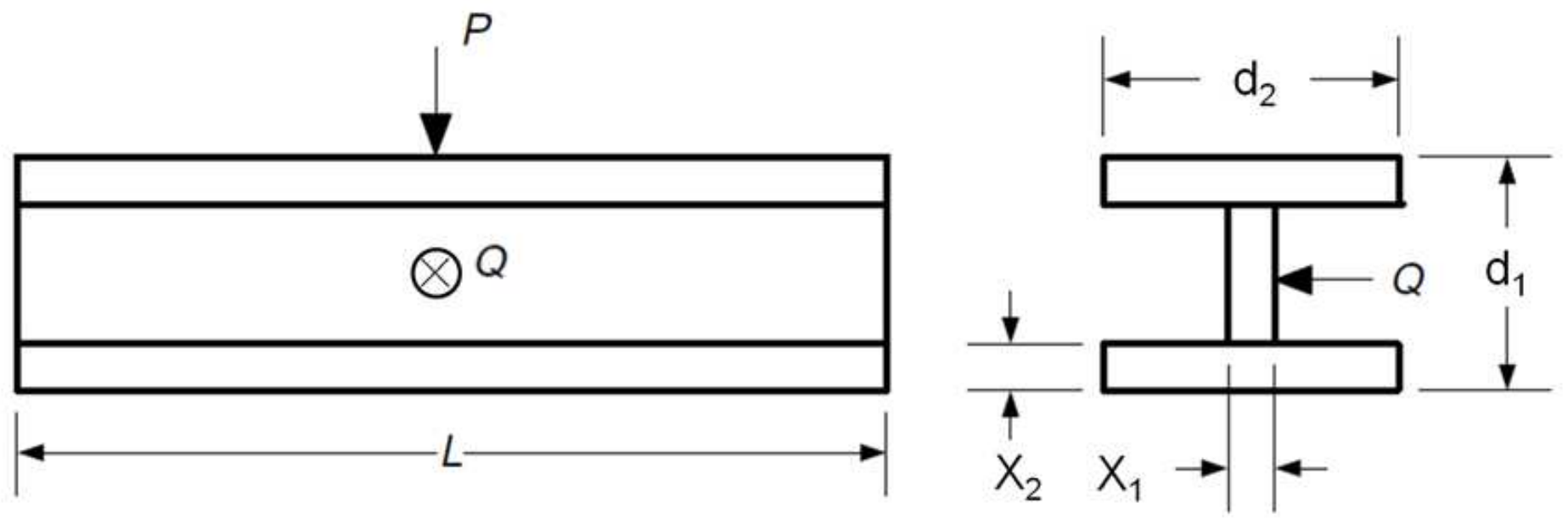

Figure 6

A beam design problem.

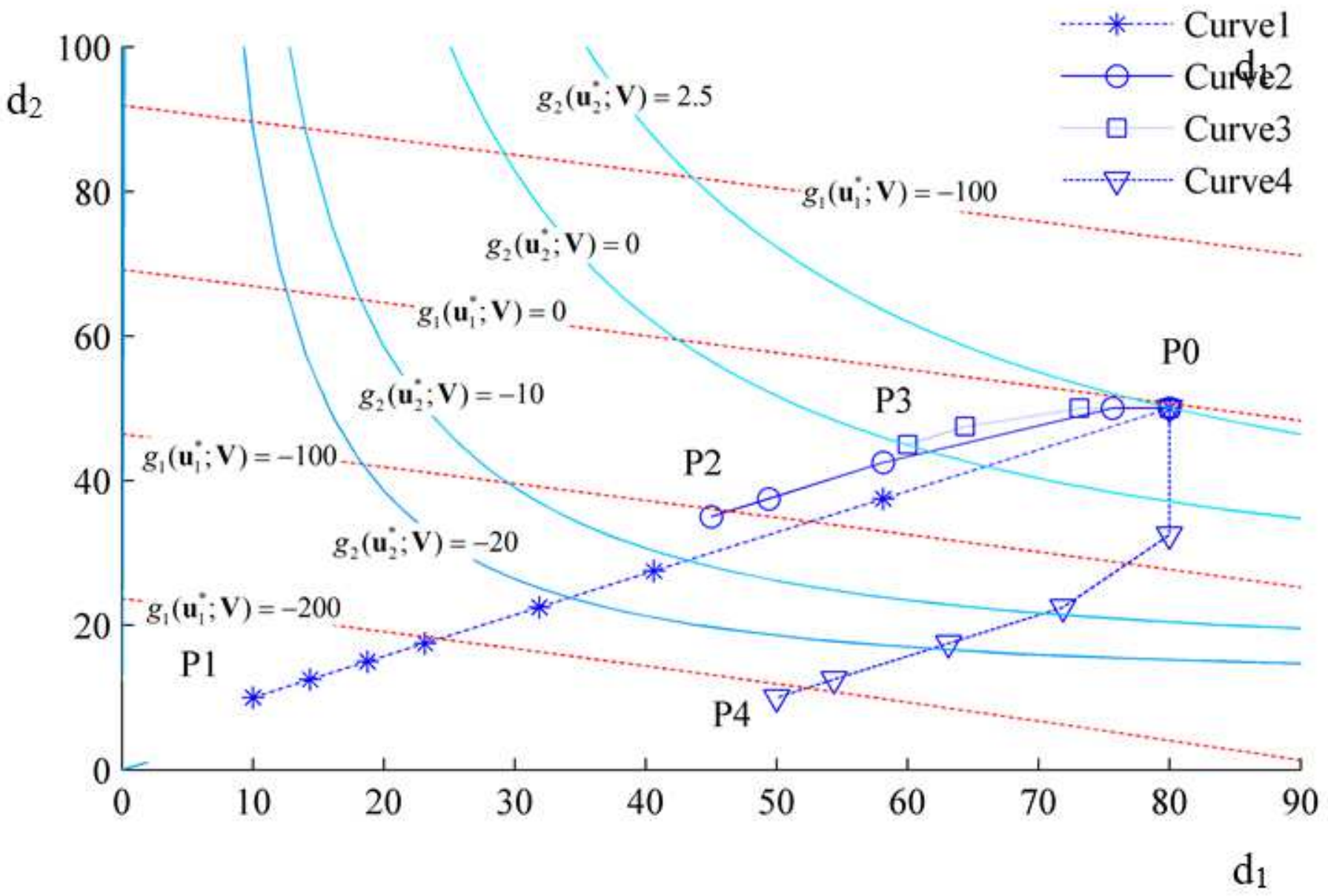

Figure 7

Iterative optimization process with different starting design points. 


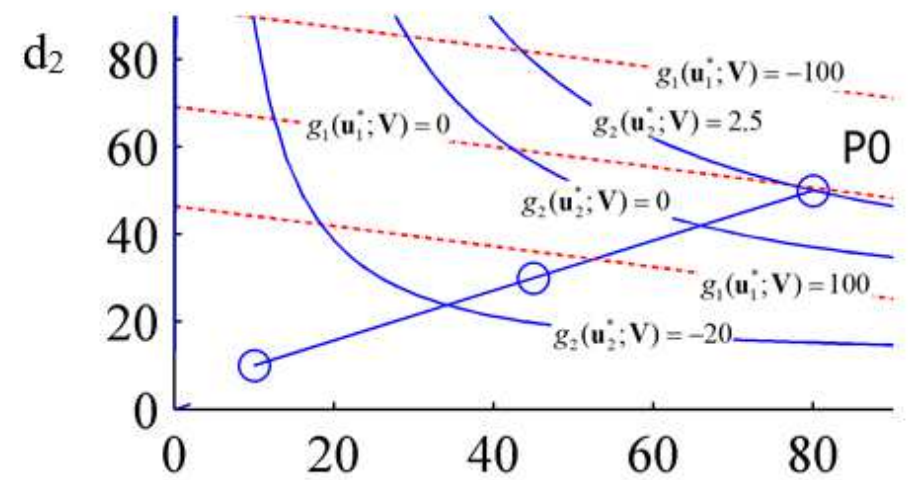

(a)

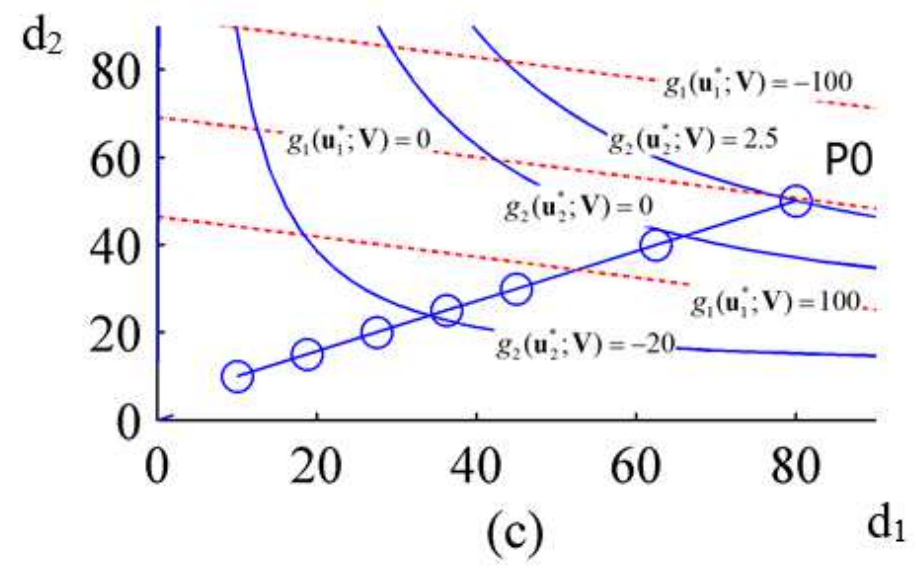

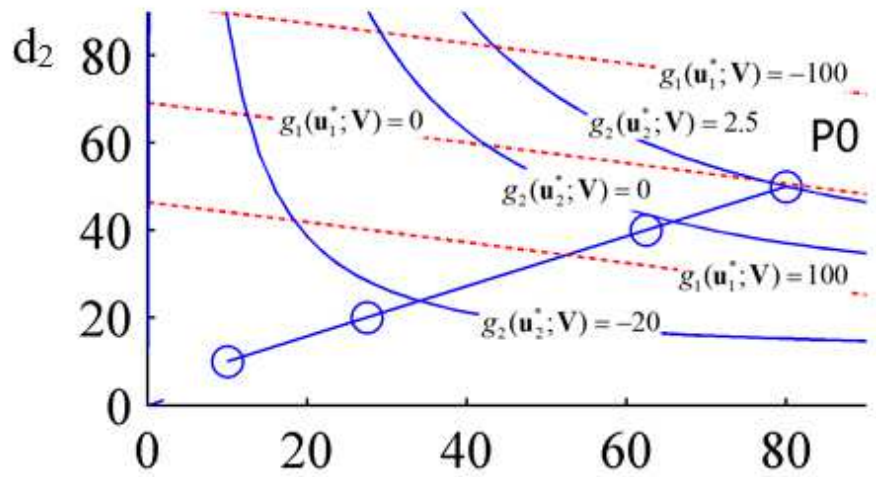

(b)

$\mathrm{d}_{1}$

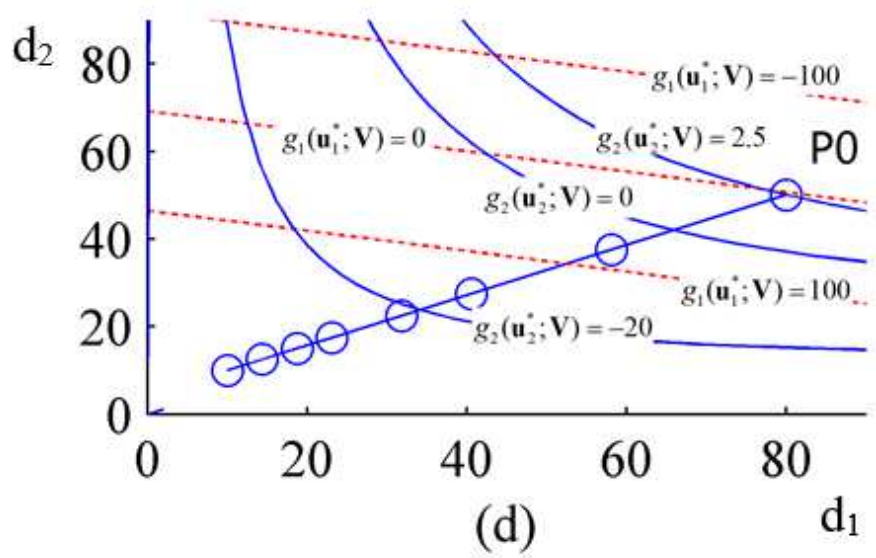

Figure 8

Iterative trajectory with different TR radii. 


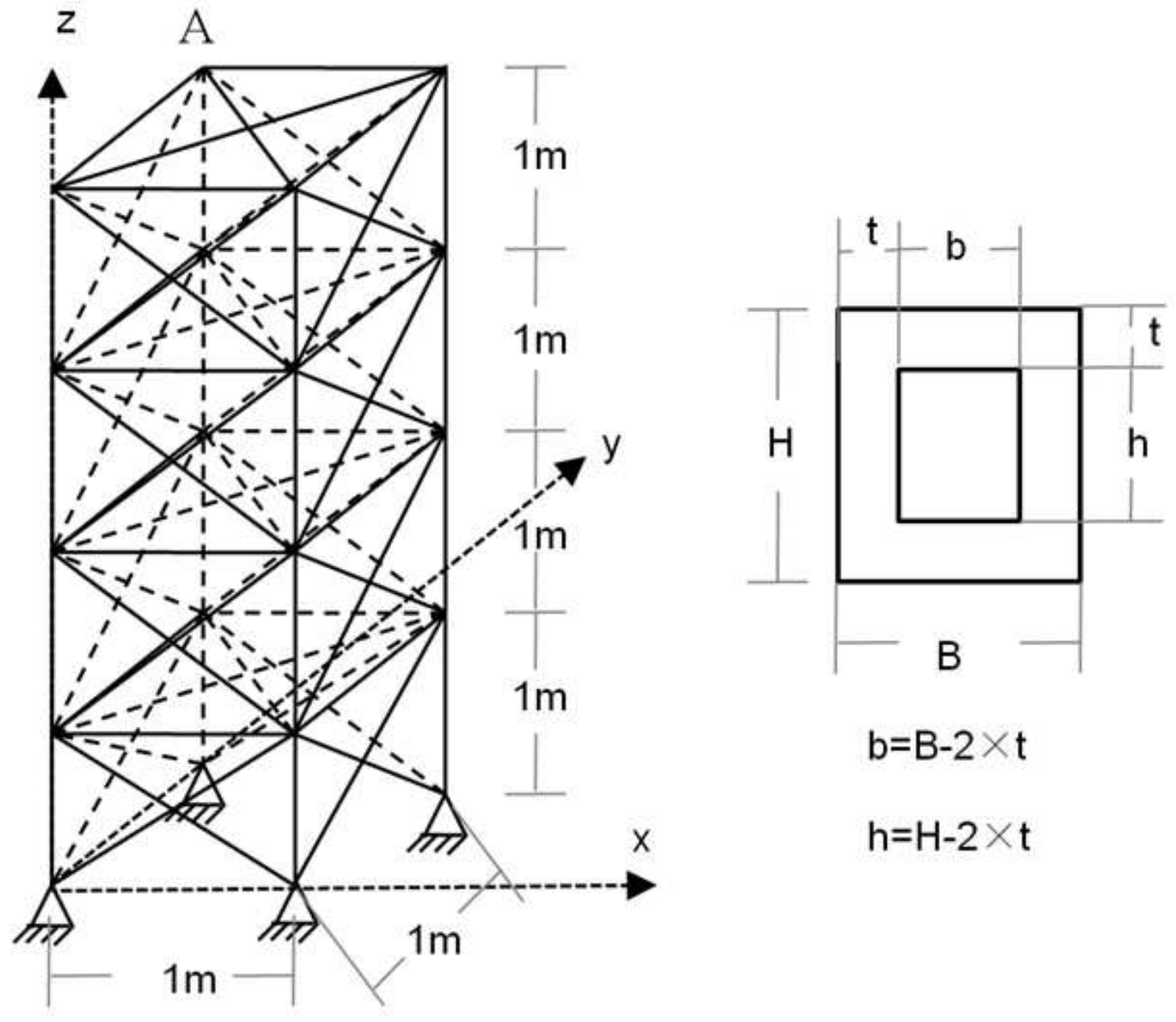

Figure 9

A 72-bar truss. 


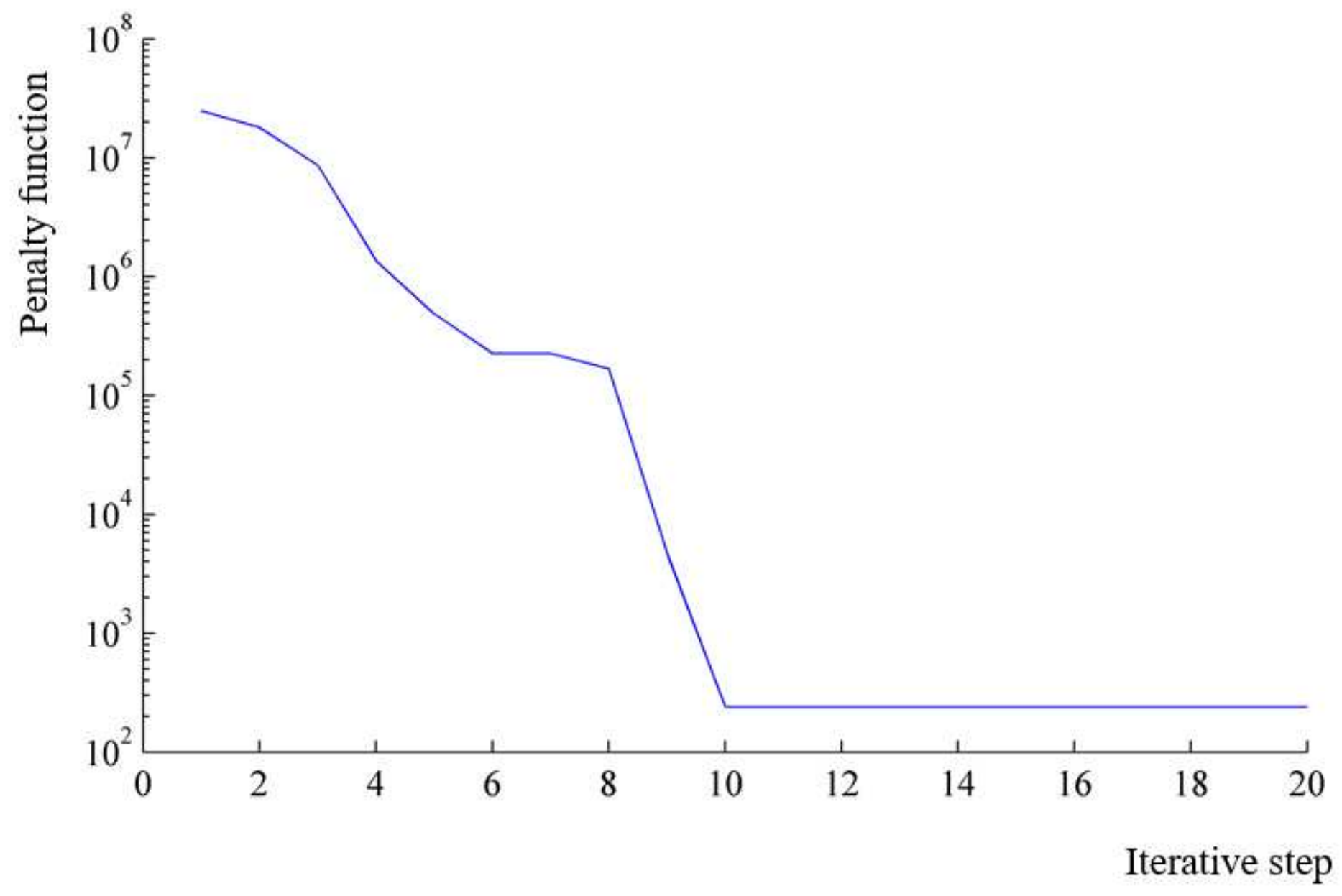

Figure 10

Iteration history of the 72-bar truss.

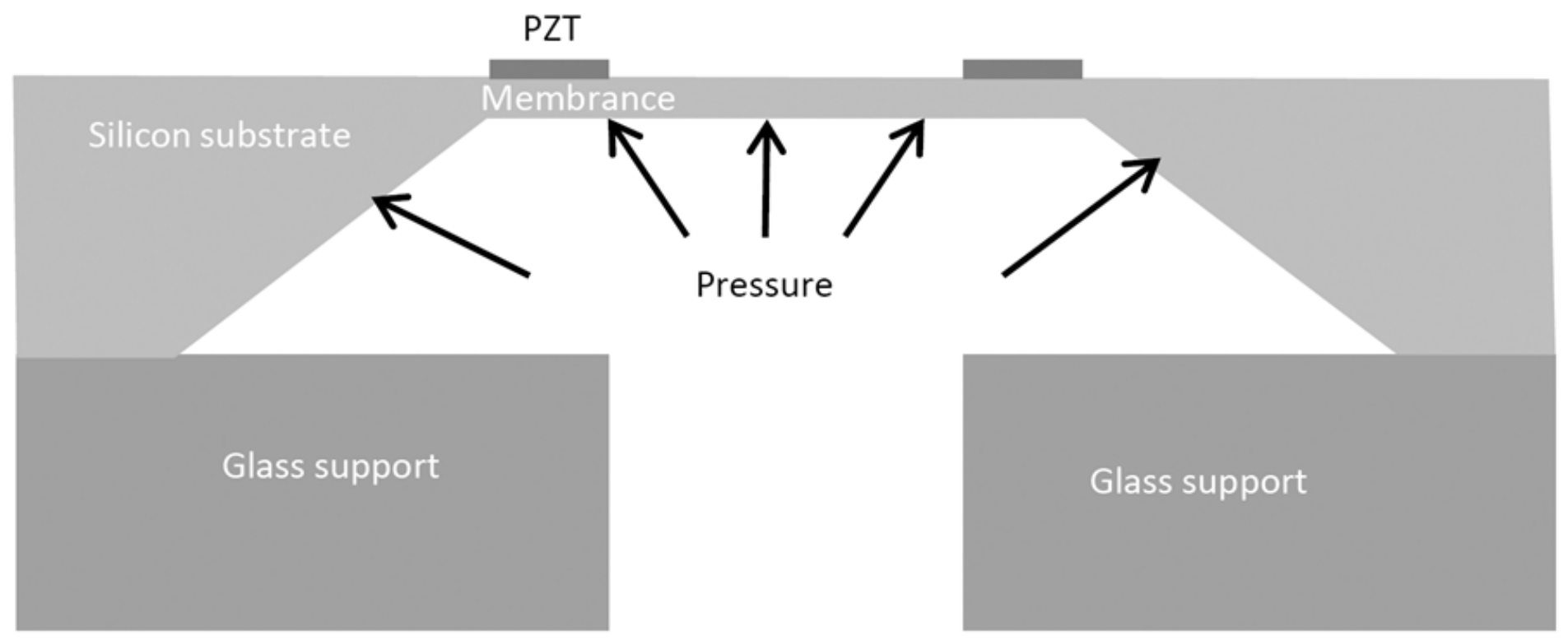


Figure 11

Schema of piezoresistive pressure sensor.

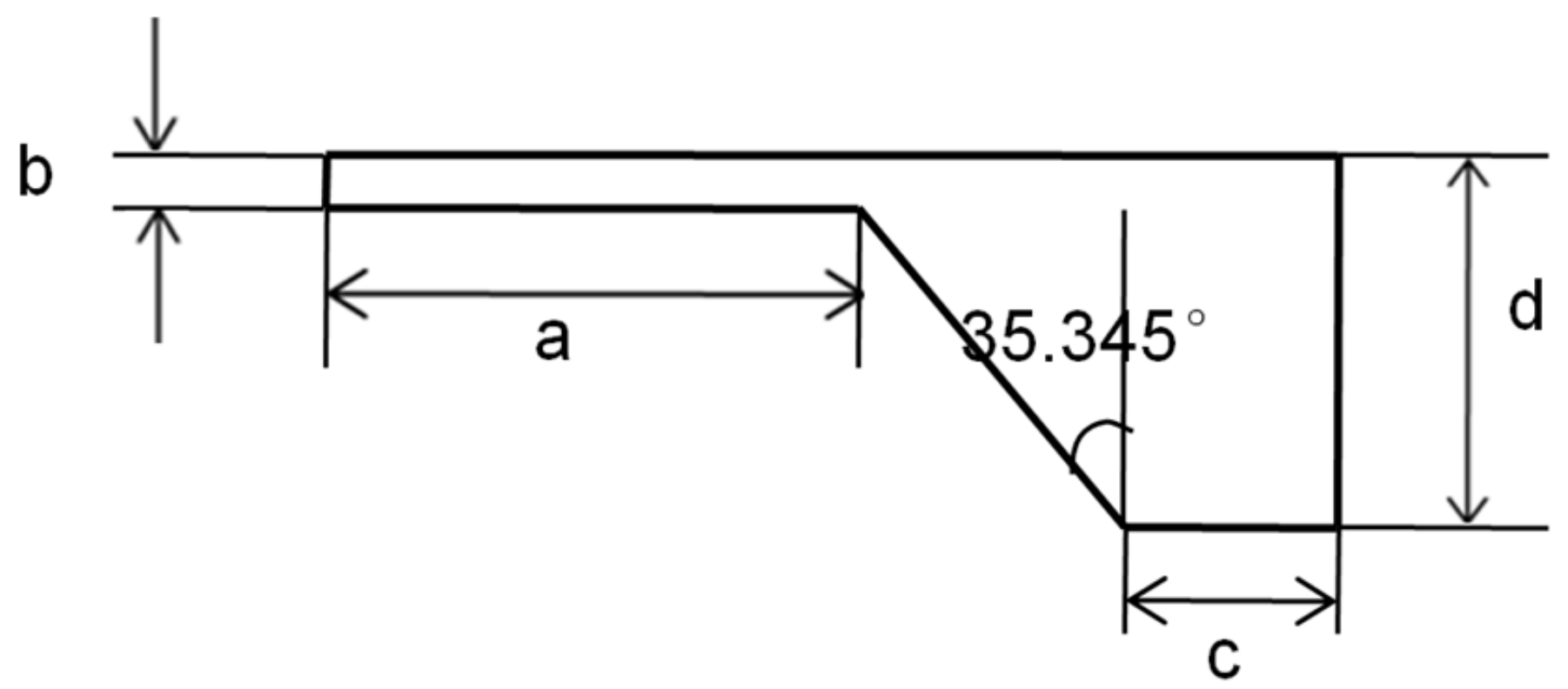

Figure 12

The main sizes of pressure sensor. 


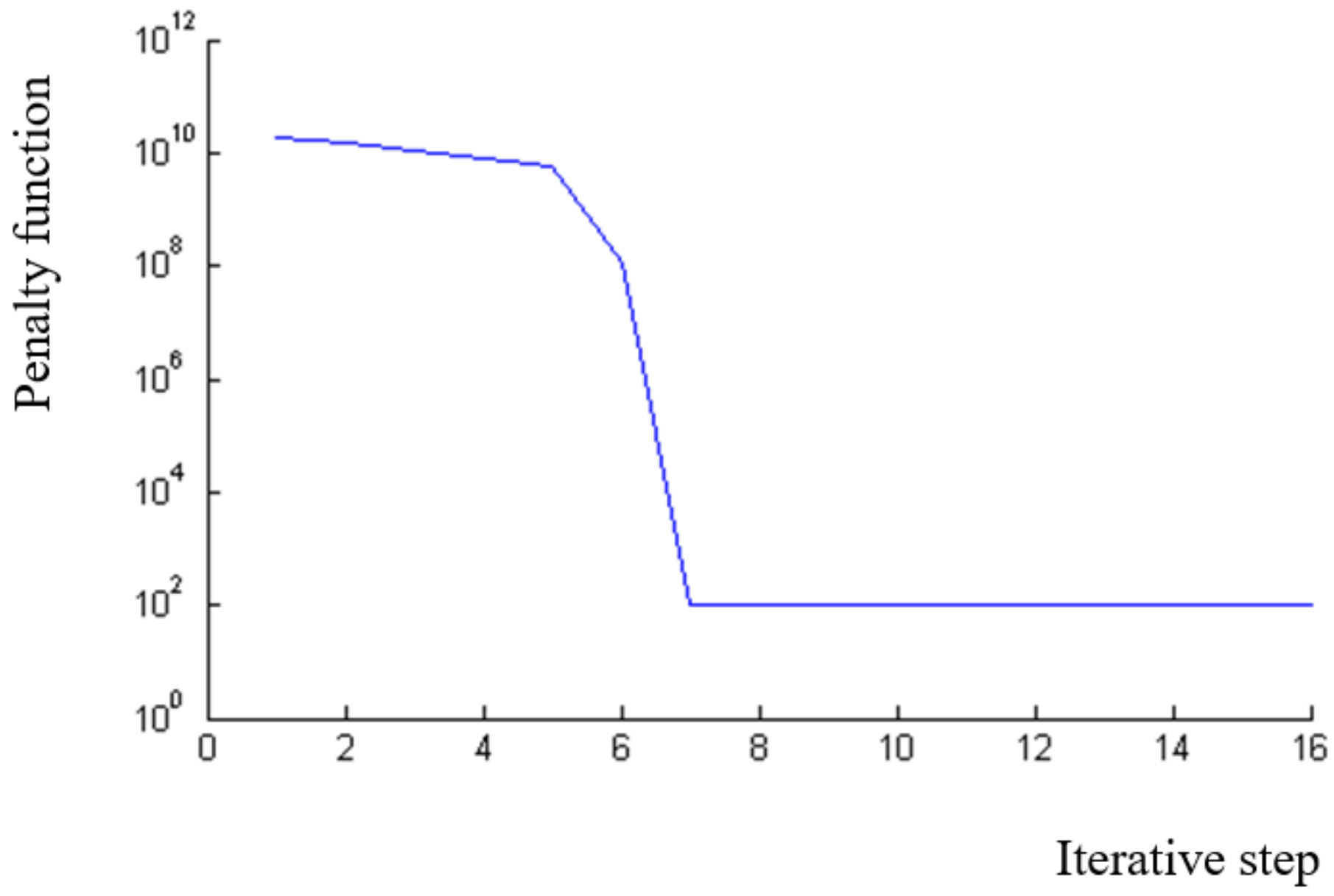

Figure 13

Iteration history of the pressure sensor design. 